\title{
A Catalyst-Free Synthesis of Phosphinic Amides using $O$-Benzoylhydroxylamines
}

\author{
Rui Zhu, Chongqing Pan and Zhenhua Gu*
}

Supporting Information

\section{General Information}

The reactions were carried out in Schlenk tubes fitted with glass stoppersunder under open air atmosphere. Flash column chromatography was performed using $40-63 \mu \mathrm{m}$ silica gel as the stationary phase. ${ }^{1} \mathrm{H},{ }^{13} \mathrm{C},{ }^{19} \mathrm{~F}$ and ${ }^{31} \mathrm{P}$ NMR spectra were recorded on a Bruker AC-400 FT spectrometer using solvent residue as an internal reference (7.26 and $77.00 \mathrm{ppm}$ for $\mathrm{CDCl}_{3}$, respectively). High resolution mass spectral analysis (HRMS) was performed on Water XEVO G2 Q-TOF (Waters Corporation).

\section{EPR experiments}

EPR spectra was recorded at $298 \mathrm{~K}$ on EPR spectrometer operated at $9.062 \mathrm{GHz}$. Typical spectrometer parameters are shown as follows, scan range: $10 \mathrm{mT}$; center field set: $324 \mathrm{mT}$; time constant: $0.1 \mathrm{~s}$; scan time: $60 \mathrm{~s}$; modulation amplitude: $0.3 \mathrm{mT}$; modulation frequency: $100 \mathrm{kHz}$; microwave power: $10 \mathrm{~mW}$.

The interaction between diphenylphosphine oxide, $N$-benzoyloxylmorpholine, and $\mathrm{K}_{2} \mathrm{CO}_{3}$ was investigated by electron paramagnetic resonance (EPR) (X band, 9.1GHz, RT): diphenylphosphine oxide ( $0.20 \mathrm{mmol}), N$-benzoyloxylmorpholine (1.2 equiv), $\mathrm{K}_{2} \mathrm{CO}_{3}$ (1.1 equiv) and $t$-AmylOH (2 $\mathrm{ml})$ were added sequentially in a schlenk tube under open air atmosphere. The reaction was stirred at $105{ }^{\circ} \mathrm{C}$ for $20 \mathrm{~min}$, then put the reaction system into liquid nitrogen. Afterwards, $60 \mu \mathrm{L}$ of the melting mixture was quickly taken out into a small tube and analyzed by EPR.
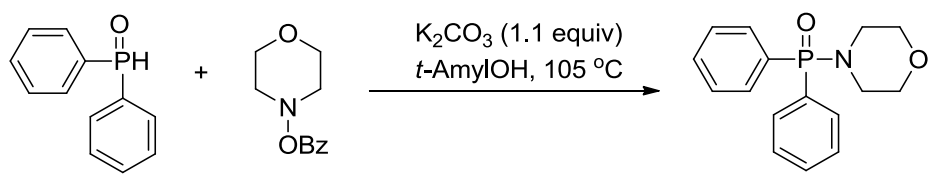

$0.20 \mathrm{mmol}$

$0.24 \mathrm{mmol}$ 


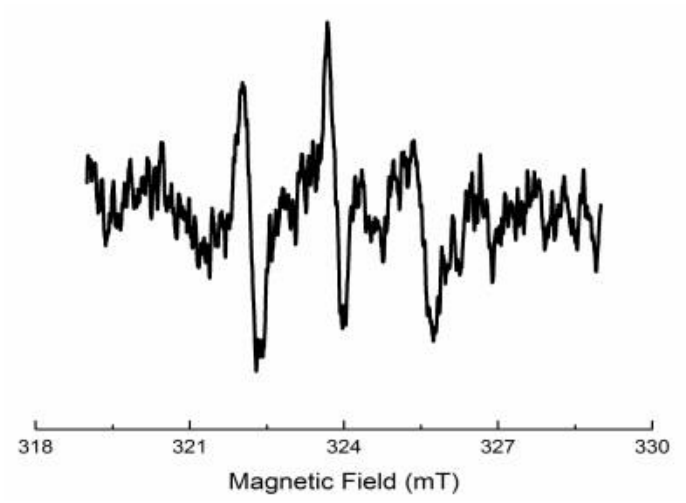

Figure S1 The EPR spectra

\section{Typical Procedures for the preparation of 3a:}<smiles>O=[PH](c1ccccc1)c1ccccc1</smiles>

1a<smiles>O=C(Oc1ccccc1)N1CCOCC1</smiles>

$2 a$

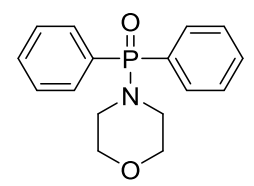

3a

$\mathrm{N}$-benzoyloxylmorpholine $2 \mathrm{a}$ ( $50 \mathrm{mg}, 0.24 \mathrm{mmol}, 1.2$ equiv) was added to a solution of $1 \mathbf{a}$ (40 mg, $0.20 \mathrm{mmol}, 1.0$ equiv) and potassium carbonate (30 mg, $0.22 \mathrm{mmol}, 1.1$ equiv) in $t$-amyl alcohol $(2.0 \mathrm{~mL})$ at room temperature. The mixture was stirred at $105{ }^{\circ} \mathrm{C}$ for $8 \mathrm{~h}$ under air atmosphere before being diluted with water $(15 \mathrm{~mL})$ and extracted with dichloromethane $(3 \times 10 \mathrm{~mL})$. The combined organic layer was washed with brine $(15 \mathrm{~mL})$, dried over sodium sulfate, filtrated and concentrated. The crude product was purified by column chromatography (ethyl acetate, then hexane/dichloromethane 40/1) to afford 3a (49 mg, 85\%) as white solid. ${ }^{1} \mathbf{H}$ NMR $\left(400 \mathrm{MHz}, \mathrm{CDCl}_{3}\right) \delta$ 7.97-7.78 (m, $\left.4 \mathrm{H}\right), 7.58-7.38(\mathrm{~m}, 6 \mathrm{H}), 3.70(\mathrm{t}, J=4.6 \mathrm{~Hz}, 4$ $\mathrm{H}), 3.6-2.99(\mathrm{~m}, 4 \mathrm{H}) ;{ }^{13} \mathbf{C}$ NMR $\left(100 \mathrm{MHz}, \mathrm{CDCl}_{3}\right) \delta 132.4$ (d, $\left.J=9.1 \mathrm{~Hz}\right), 131.9$ (d, $J=2.6$ $\mathrm{Hz}), 130.8(\mathrm{~d}, J=128.3 \mathrm{~Hz}), 128.7(\mathrm{~d}, J=12.3 \mathrm{~Hz}), 67.2(\mathrm{~d}, J=6.7 \mathrm{~Hz}), 44.9 ;{ }^{31} \mathbf{P}$ NMR $(162$ $\left.\mathrm{MHz}, \mathrm{CDCl}_{3}\right) \delta$ 29.08; HRMS (ESI) calcd. for $\mathrm{C}_{16} \mathrm{H}_{19} \mathrm{NO}_{2} \mathrm{P}[\mathrm{M}+\mathrm{H}]^{+}:$288.1153, found 288.1154 .

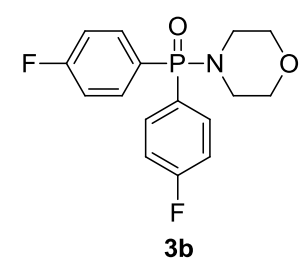

3b $(52 \mathrm{mg}, 81 \%$, reaction time: $6 \mathrm{~h})\left(\mathrm{R}_{\mathrm{f}} 0.4\right.$, EA) was prepared following the typical procedure in $0.20 \mathrm{mmol}$ scale as colorless oil. ${ }^{1} \mathbf{H}$ NMR $\left(400 \mathrm{MHz}, \mathrm{CDCl}_{3}\right) \delta 8.01-7.70(\mathrm{~m}, 4$ $\mathrm{H}), 7.26-7.03(\mathrm{~m}, 4 \mathrm{H}), 3.69(\mathrm{t}, J=4.6 \mathrm{~Hz}, 4 \mathrm{H}), 3.15-2.96(\mathrm{~m}, 4 \mathrm{H}) ;{ }^{13} \mathbf{C}$ NMR $(100 \mathrm{MHz}$, $\left.\mathrm{CDCl}_{3}\right) \delta 165.2\left(\mathrm{dd}, J=252.5\left(\mathrm{C}_{\mathrm{C}, \mathrm{F}}\right), 3.3 \mathrm{~Hz}\right), 134.8(\mathrm{dd}, J=10.4,8.8 \mathrm{~Hz}), 126.7(\mathrm{dd}, J=$ 132.7, $\left.3.4\left(\mathrm{C}_{\mathrm{C}, \mathrm{F}}\right) \mathrm{Hz}\right), 116.2(\mathrm{dd}, J=21.2,13.6 \mathrm{~Hz}), 67.2(\mathrm{~d}, J=6.6 \mathrm{~Hz}), 45.0 ;{ }^{19} \mathbf{F}$ NMR $(376$ $\left.\mathrm{MHz}, \mathrm{CDCl}_{3}\right) \delta-106.43 ;{ }^{31} \mathbf{P}$ NMR $\left(162 \mathrm{MHz}, \mathrm{CDCl}_{3}\right) \delta$ 27.26; HRMS (ESI) calcd. for 
$\mathrm{C}_{16} \mathrm{H}_{17} \mathrm{~F}_{2} \mathrm{NO}_{2} \mathrm{P}[\mathrm{M}+\mathrm{H}]^{+}: 324.0965$, found 324.0966.

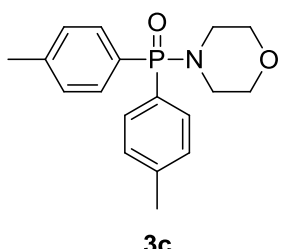

3c (49 mg, 78\%, reaction time: $10 \mathrm{~h})\left(\mathrm{R}_{\mathrm{f}} 0.3\right.$, EA) was prepared following the typical procedure in $0.20 \mathrm{mmol}$ scale as yellow oil. ${ }^{1} \mathbf{H}$ NMR $\left(400 \mathrm{MHz}, \mathrm{CDCl}_{3}\right) \delta$ 7.82-7.65 (m, 4 H), 7.32-7.18 (m, 4 H), 3.68 (t, $J=4.4 \mathrm{~Hz}, 4 \mathrm{H}), 3.19-2.91(\mathrm{~m}, 4 \mathrm{H}), 2.35$ (s, $6 \mathrm{H}) ;{ }^{13} \mathbf{C}$ NMR $\left(100 \mathrm{MHz}, \mathrm{CDCl}_{3}\right) \delta 142.3(\mathrm{~d}, J=2.8 \mathrm{~Hz}), 132.3(\mathrm{~d}, J=9.4 \mathrm{~Hz}), 129.4(\mathrm{~d}, J=12.8 \mathrm{~Hz})$, $127.7(\mathrm{~d}, J=130.8 \mathrm{~Hz}), 67.2(\mathrm{~d}, J=6.8 \mathrm{~Hz}), 44.9,21.5 ;{ }^{31} \mathbf{P} \mathbf{N M R}\left(162 \mathrm{MHz}, \mathrm{CDCl}_{3}\right) \delta 29.58$; HRMS (ESI) calcd. for $\mathrm{C}_{18} \mathrm{H}_{23} \mathrm{NO}_{2} \mathrm{P}[\mathrm{M}+\mathrm{H}]^{+}:$316.1466, found 316.1463 .

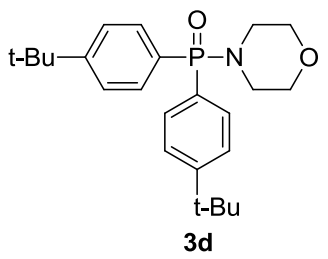

3d $(61 \mathrm{mg}, 88 \%$, reaction time: $8 \mathrm{~h})\left(\mathrm{R}_{\mathrm{f}} 0.4\right.$, EA) was prepared following the typical procedure in $0.20 \mathrm{mmol}$ scale as yellow solid. ${ }^{1} \mathbf{H}$ NMR $\left(400 \mathrm{MHz}, \mathrm{CDCl}_{3}\right) \delta 7.84-7.72(\mathrm{~m}, 4$ H), 7.50-7.41 (m, $4 \mathrm{H}), 3.69$ (t, $J=4.4 \mathrm{~Hz}, 4 \mathrm{H}), 3.19-2.95(\mathrm{~m}, 4 \mathrm{H}), 1.29(\mathrm{~s}, 18 \mathrm{H}) ;{ }^{13} \mathbf{C}$ NMR $\left(100 \mathrm{MHz}, \mathrm{CDCl}_{3}\right) \delta 155.2(\mathrm{~d}, J=2.7 \mathrm{~Hz}), 132.2(\mathrm{~d}, J=9.4 \mathrm{~Hz}), 127.8(\mathrm{~d}, J=130.6 \mathrm{~Hz})$, $125.60(\mathrm{~d}, J=12.5 \mathrm{~Hz}), 67.25(\mathrm{~d}, J=6.9 \mathrm{~Hz}), 44.89,34.90,31.05 ;{ }^{31} \mathbf{P}$ NMR $(162 \mathrm{MHz}$, $\left.\mathrm{CDCl}_{3}\right) \delta 29.25$; HRMS (ESI) calcd. for $\mathrm{C}_{24} \mathrm{H}_{35} \mathrm{NO}_{2} \mathrm{P}[\mathrm{M}+\mathrm{H}]^{+}: 400.2405$, found 400.2404.

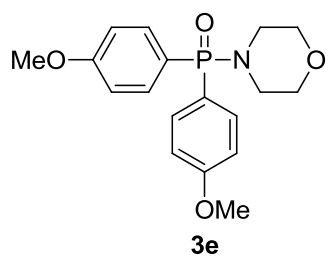

3e $(61 \mathrm{mg}, 88 \%$, reaction time: $10 \mathrm{~h})\left(\mathrm{R}_{\mathrm{f}} 0.3\right.$, EA) was prepared following the typical procedure in $0.20 \mathrm{mmol}$ scale as yellow oil. ${ }^{1} \mathbf{H}$ NMR $\left(400 \mathrm{MHz}, \mathrm{CDCl}_{3}\right) \delta$ 7.87-7.69 (m, 4 H), 7.00-6.91 (m, 4 H), 3.83 (s, $6 \mathrm{H}), 3.69$ (t, $J=4.4 \mathrm{~Hz}, 4 \mathrm{H}), 3.11-2.99(\mathrm{~m}, 4 \mathrm{H}) ;{ }^{13} \mathbf{C}$ NMR $\left(100 \mathrm{MHz}, \mathrm{CDCl}_{3}\right) \delta 162.5,134.1(\mathrm{~d}, J=10.4 \mathrm{~Hz}), 122.4(\mathrm{~d}, J=135.2 \mathrm{~Hz}), 114.2(\mathrm{~d}, J=13.3$ $\mathrm{Hz}), 67.3(\mathrm{~d}, J=6.9 \mathrm{~Hz}), 55.3,44.9 ;{ }^{31} \mathbf{P}$ NMR $\left(162 \mathrm{MHz}, \mathrm{CDCl}_{3}\right) \delta 29.27$; HRMS (ESI) calcd. for $\mathrm{C}_{18} \mathrm{H}_{23} \mathrm{NO}_{4} \mathrm{P}[\mathrm{M}+\mathrm{H}]^{+}: 348.1365$, found 348.1368 .

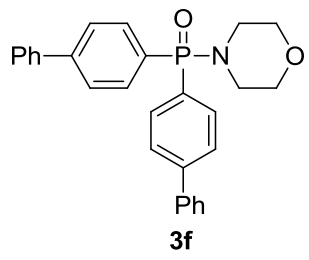


3f $(74 \mathrm{mg}, 84 \%$, reaction time: $12 \mathrm{~h})\left(\mathrm{R}_{\mathrm{f}} 0.3\right.$, EA) was prepared following the typical procedure in $0.20 \mathrm{mmol}$ scale as yellow oil. ${ }^{1} \mathbf{H} \mathbf{~ N M R}\left(400 \mathrm{MHz}, \mathrm{CDCl}_{3}\right) \delta 7.98(\mathrm{dd}, J=11.2$, $8.0 \mathrm{~Hz}, 4 \mathrm{H}), 7.70(\mathrm{dd}, J=8.4,3.2 \mathrm{~Hz}, 4 \mathrm{H}), 7.66-7.55(\mathrm{~m}, 4 \mathrm{H}), 7.54-7.41(\mathrm{~m}, 4 \mathrm{H})$, 7.41-7.33 (m, $2 \mathrm{H}), 3.76$ (t, $J=4.6 \mathrm{~Hz}, 4 \mathrm{H}), 3.25-3.06$ (m, $4 \mathrm{H}) ;{ }^{13} \mathbf{C} \mathbf{N M R}\left(100 \mathrm{MHz}, \mathrm{CDCl}_{3}\right)$ $\delta 144.8(\mathrm{~d}, J=2.8 \mathrm{~Hz}), 139.8,132.87(\mathrm{~d}, J=9.4 \mathrm{~Hz}), 129.3(\mathrm{~d}, J=130.4 \mathrm{~Hz}), 128.9,128.1$, 127.5, 127.4, $67.2(\mathrm{~d}, J=6.6 \mathrm{~Hz}), 45.0 ;{ }^{31} \mathbf{P}$ NMR $\left(162 \mathrm{MHz}, \mathrm{CDCl}_{3}\right) \delta 29.11$; HRMS (ESI) calcd. for $\mathrm{C}_{28} \mathrm{H}_{27} \mathrm{NO}_{2} \mathrm{P}[\mathrm{M}+\mathrm{H}]^{+}:$440.1779, found 440.1783 .

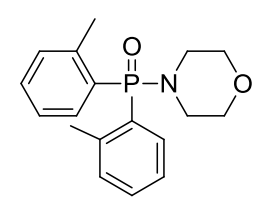

$3 \mathbf{g}$

$3 \mathrm{~g}$ (37 mg, 59\%, reaction time: $15 \mathrm{~h})\left(\mathrm{R}_{\mathrm{f}} 0.3\right.$, EA) was prepared following the typical procedure in $0.20 \mathrm{mmol}$ scale as colorless oil. ${ }^{1} \mathbf{H}$ NMR $\left(400 \mathrm{MHz}, \mathrm{CDCl}_{3}\right) \delta$ 7.50-7.36 (m, 4 H), 7.31-7.16 (m, $4 \mathrm{H}), 3.71(\mathrm{t}, J=4.4 \mathrm{~Hz}, 4 \mathrm{H}), 3.26-3.09(\mathrm{~m}, 4 \mathrm{H}), 2.53$ (s, $6 \mathrm{H}) ;{ }^{13} \mathbf{C ~ N M R}$ $\left(100 \mathrm{MHz}, \mathrm{CDCl}_{3}\right) \delta 143.0(\mathrm{~d}, J=9.1 \mathrm{~Hz}), 132.7(\mathrm{~d}, J=11.5 \mathrm{~Hz}), 132.05,131.90(\mathrm{t}, J=3.2$ $\mathrm{Hz}), 130.0$ (d, $J=122.1 \mathrm{~Hz}), 125.39$ (d, $J=12.9 \mathrm{~Hz}), 67.36$ (d, $J=5.3 \mathrm{~Hz}), 44.96,21.58$ (d, $J$ $=3.7 \mathrm{~Hz}) ;{ }^{31} \mathbf{P}$ NMR $\left(162 \mathrm{MHz}, \mathrm{CDCl}_{3}\right) \delta 34.60$; HRMS (ESI) calcd. for $\mathrm{C}_{18} \mathrm{H}_{23} \mathrm{NO}_{2} \mathrm{P}[\mathrm{M}+$ $\mathrm{H}]^{+}: 316.1466$, found 316.1465 .

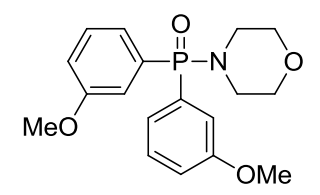

$3 h$

3h (47 mg, 68\%, reaction time: $12 \mathrm{~h})\left(\mathrm{R}_{\mathrm{f}} 0.3\right.$, EA) was prepared following the typical procedure in $0.20 \mathrm{mmol}$ scale as yellow oil. ${ }^{1} \mathbf{H}$ NMR $\left(400 \mathrm{MHz}, \mathrm{CDCl}_{3}\right) \delta$ 7.48-7.32 (m, 6 H), 7.09-6.98 (m, $2 \mathrm{H}), 3.83$ (s, $6 \mathrm{H}), 3.70$ (t, $J=4.4 \mathrm{~Hz}, 4 \mathrm{H}), 3.15-3.01(\mathrm{~m}, 4 \mathrm{H}) ;{ }^{13} \mathbf{C}$ NMR $\left(100 \mathrm{MHz}, \mathrm{CDCl}_{3}\right) \delta 159.7(\mathrm{~d}, J=15.6 \mathrm{~Hz}), 132.2(\mathrm{~d}, J=127.8 \mathrm{~Hz}), 129.9(\mathrm{~d}, J=14.6 \mathrm{~Hz})$, $124.3(\mathrm{~d}, J=8.7 \mathrm{~Hz}), 118.1(\mathrm{~d}, J=2.7 \mathrm{~Hz}), 117.3(\mathrm{~d}, J=10.3 \mathrm{~Hz}), 67.2(\mathrm{~d}, J=6.7 \mathrm{~Hz}), 55.4$, 45.0; ${ }^{31} \mathbf{P}$ NMR $\left(162 \mathrm{MHz}, \mathrm{CDCl}_{3}\right) \delta 29.16$; HRMS (ESI) calcd. for $\mathrm{C}_{18} \mathrm{H}_{23} \mathrm{NO}_{4} \mathrm{P}[\mathrm{M}+\mathrm{H}]^{+}$: 348.1365 , found 348.1370 .

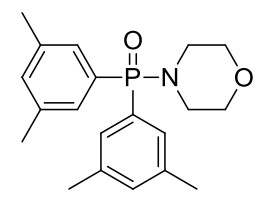

$3 \mathbf{i}$

3i $(57 \mathrm{mg}, 83 \%$, reaction time: $12 \mathrm{~h})\left(\mathrm{R}_{\mathrm{f}} 0.3\right.$, EA) was prepared following the typical procedure in $0.20 \mathrm{mmol}$ scale as yellow oil. ${ }^{1} \mathbf{H}$ NMR $\left(400 \mathrm{MHz}, \mathrm{CDCl}_{3}\right) \delta 7.46(\mathrm{bd}, J=11.6$ $\mathrm{Hz}, 4 \mathrm{H}), 7.11$ (bs, $2 \mathrm{H}), 3.70(\mathrm{t}, J=4.6 \mathrm{~Hz}, 4 \mathrm{H}), 3.14-2.96(\mathrm{~m}, 4 \mathrm{H}), 2.33(\mathrm{~s}, 12 \mathrm{H}) ;{ }^{13} \mathrm{C}$ NMR $\left(100 \mathrm{MHz}, \mathrm{CDCl}_{3}\right) \delta 138.3(\mathrm{~d}, J=12.9 \mathrm{~Hz}), 133.6(\mathrm{~d}, J=2.9 \mathrm{~Hz}), 130.7$ (d, $J=127.0$ 
$\mathrm{Hz}), 129.9(\mathrm{~d}, J=9.0 \mathrm{~Hz}), 67.25(\mathrm{~d}, J=6.5 \mathrm{~Hz}), 45.01,21.27 ;{ }^{31} \mathbf{P}$ NMR $\left(162 \mathrm{MHz}, \mathrm{CDCl}_{3}\right) \delta$ 30.02; HRMS (ESI) calcd. for $\mathrm{C}_{20} \mathrm{H}_{27} \mathrm{NO}_{2} \mathrm{P}[\mathrm{M}+\mathrm{H}]^{+}: 344.1779$, found 344.1786.

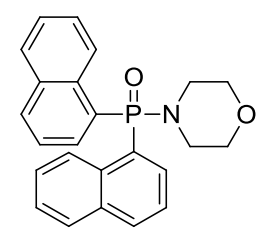

3j

3j $(58 \mathrm{mg}, 75 \%$, reaction time: $12 \mathrm{~h})\left(\mathrm{R}_{\mathrm{f}} 0.4\right.$, EA) was prepared following the typical procedure in $0.20 \mathrm{mmol}$ scale as yellow solid. ${ }^{1} \mathbf{H}$ NMR $\left(400 \mathrm{MHz}, \mathrm{CDCl}_{3}\right) \delta$ 9.01-8.79 (m, 2 H), 8.01 (d, $J=8.4$ Hz, 2 H), 7.92-7.82 (m, 2 H), 7.71-7.47 (m, $6 \mathrm{H})$, 7.47-7.34 (m, $2 \mathrm{H}), 3,67$ (t, $J=4.4 \mathrm{~Hz}, 4 \mathrm{H}), 3.37-3.20(\mathrm{~m}, 4 \mathrm{H}) ;{ }^{13} \mathbf{C} \mathbf{~ N M R}\left(100 \mathrm{MHz}, \mathrm{CDCl}_{3}\right) \delta 133.9$ (dd, $J=9.7$, $6.9 \mathrm{~Hz}), 133.3(\mathrm{~d}, J=2.9 \mathrm{~Hz}), 133.1(\mathrm{~d}, J=11.4 \mathrm{~Hz}), 128.7(\mathrm{~d}, J=1.0 \mathrm{~Hz}), 128.7,127.7$, $127.6(\mathrm{~d}, J=4.6 \mathrm{~Hz}), 127.5,127.0(\mathrm{~d}, J=85.4 \mathrm{~Hz}), 124.4(\mathrm{~d}, J=14.9 \mathrm{~Hz}), 67.3(\mathrm{~d}, J=4.9$ $\mathrm{Hz}), 45.1 ;{ }^{31} \mathbf{P}$ NMR $\left(162 \mathrm{MHz}, \mathrm{CDCl}_{3}\right) \delta 35.65$; HRMS (ESI) calcd. for $\mathrm{C}_{24} \mathrm{H}_{23} \mathrm{NO}_{2} \mathrm{P}[\mathrm{M}+$ $\mathrm{H}]^{+}: 388.1466$, found 388.1469 .

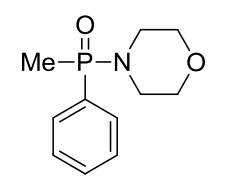

$3 \mathbf{k}$

3k $(29 \mathrm{mg}, 64 \%$, reaction time: $9 \mathrm{~h})\left(\mathrm{R}_{\mathrm{f}} 0.2\right.$, EA) was prepared following the typical procedure in $0.20 \mathrm{mmol}$ scale as colorless oil. ${ }^{1} \mathbf{H}$ NMR $\left(400 \mathrm{MHz}, \mathrm{CDCl}_{3}\right) \delta 7.85-7.71(\mathrm{~m}, 2$ $\mathrm{H})$, 7.64-7.35 (m, $3 \mathrm{H}), 3.75-3.58(\mathrm{~m}, 4 \mathrm{H}), 3.15-2.93(\mathrm{~m}, 4 \mathrm{H}), 1.71(\mathrm{~d}, J=13.6 \mathrm{~Hz}, 3 \mathrm{H}) ;{ }^{13} \mathbf{C}$ NMR $\left(100 \mathrm{MHz}, \mathrm{CDCl}_{3}\right) \delta 132.1(\mathrm{~d}, J=123.5 \mathrm{~Hz}), 132.0(\mathrm{~d}, J=2.7 \mathrm{~Hz}), 131.3(\mathrm{~d}, J=9.5$ $\mathrm{Hz}), 128.7(\mathrm{~d}, J=12.3 \mathrm{~Hz}), 67.1(\mathrm{~d}, J=7.0 \mathrm{~Hz}), 44.2,13.8(\mathrm{~d}, J=92.3 \mathrm{~Hz}) ;{ }^{31} \mathbf{P}$ NMR $(162$ $\mathrm{MHz}, \mathrm{CDCl}_{3}$ ) $\delta$ 35.43; HRMS (ESI) calcd. for $\mathrm{C}_{11} \mathrm{H}_{17} \mathrm{NO}_{2} \mathrm{P}[\mathrm{M}+\mathrm{H}]^{+}:$226.0997, found 226.1000 .

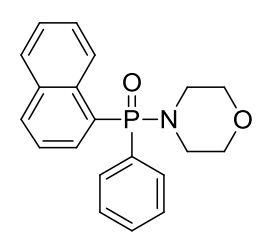

31

3l (49 mg, 72\%, reaction time: $10 \mathrm{~h})\left(\mathrm{R}_{\mathrm{f}} 0.4\right.$, EA) was prepared following the typical procedure in $0.20 \mathrm{mmol}$ scale as wihte solid. ${ }^{1} \mathbf{H} \mathbf{~ N M R}\left(300 \mathrm{MHz}, \mathrm{CDCl}_{3}\right) \delta 8.88(\mathrm{~d}, J=8.4$ $\mathrm{Hz}, 1 \mathrm{H}), 7.98(\mathrm{~d}, J=8.1 \mathrm{~Hz}, 1 \mathrm{H}), 7.93-7.73(\mathrm{~m}, 4 \mathrm{H}), 7.64-7.34(\mathrm{~m}, 6 \mathrm{H}), 3.69(\mathrm{t}, J=4.5 \mathrm{~Hz}$, 4H), 3.26-3.05 (m, $4 \mathrm{H}) ;{ }^{13} \mathbf{C}$ NMR $\left(75 \mathrm{MHz}, \mathrm{CDCl}_{3}\right) \delta 133.83(\mathrm{~d}, J=13.8 \mathrm{~Hz}), 133.78133 .6$ $(\mathrm{t}, J=11.3 \mathrm{~Hz}), 133.3(\mathrm{~d}, J=3.0 \mathrm{~Hz}), 132.3,132.2(\mathrm{~d}, J=3.7 \mathrm{~Hz}), 131.9(\mathrm{~d}, J=3.7 \mathrm{~Hz})$, $129.3(\mathrm{~d}, J=272.0 \mathrm{~Hz}), 128.8(\mathrm{~d}, J=1.7 \mathrm{~Hz}), 128.6(\mathrm{~d}, J=16.7 \mathrm{~Hz}), 126.9(\mathrm{~d}, J=95.1 \mathrm{~Hz})$, 
$126.8(\mathrm{~d}, J=5.6 \mathrm{~Hz}), 126.3,124.4(\mathrm{~d}, J=19.3 \mathrm{~Hz}), 67.2(\mathrm{~d}, J=5.9 \mathrm{~Hz}), 45.0 ;{ }^{31} \mathbf{P}$ NMR $(121$ $\left.\mathrm{MHz}, \mathrm{CDCl}_{3}\right) \delta 32.40$; HRMS (ESI) calcd. for $\mathrm{C}_{20} \mathrm{H}_{21} \mathrm{NO}_{2} \mathrm{P}[\mathrm{M}+\mathrm{H}]^{+}: 338.1310$, found 338.1309 .

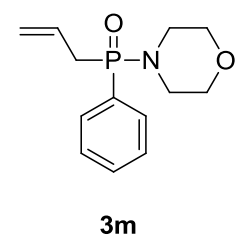

3m (45 mg, 90\%, reaction time: $9 \mathrm{~h})\left(\mathrm{R}_{\mathrm{f}} 0.3\right.$, EA) was prepared following the typical procedure in $0.20 \mathrm{mmol}$ scale as colorless oil. ${ }^{1} \mathbf{H}$ NMR $\left(400 \mathrm{MHz}, \mathrm{CDCl}_{3}\right) \delta$ 7.87-7.67 (m, 2 H), 7.61-7.41 (m, $3 \mathrm{H})$, 6.95-6.70 (m, 0.1 H), 5.98-5.84 (m, 0.2 H), 5.84-5.67 (m, 0.9 H), 5.24-5.01 (m, $1.8 \mathrm{H}), 3.67(\mathrm{t}, J=4.6 \mathrm{~Hz}, 4 \mathrm{H}), 3.18-2.99(\mathrm{~m}, 4 \mathrm{H}), 2.95-2.83(\mathrm{~m}, 1.7 \mathrm{H})$, 1.94-1.88 (m, 0.3 H); ${ }^{13} \mathbf{C}$ NMR $\left(100 \mathrm{MHz}, \mathrm{CDCl}_{3}\right) \delta 132.0(\mathrm{~d}, J=2.7 \mathrm{~Hz}), 131.8(\mathrm{dd}, J=9.2$, $4.7 \mathrm{~Hz}), 131.1$ (d, $J=121.4 \mathrm{~Hz}), 128.6(\mathrm{dd}, J=12.2,4.8 \mathrm{~Hz}), 127.7(\mathrm{~d}, J=8.6 \mathrm{~Hz}), 120.24$ (d, $J=12.4 \mathrm{~Hz}), 67.07(\mathrm{~d}, J=6.6 \mathrm{~Hz}), 44.43(\mathrm{~d}, J=4.5 \mathrm{~Hz}), 33.7(\mathrm{~d}, J=87.5 \mathrm{~Hz}) ;{ }^{31} \mathbf{P} \mathbf{~ N M R}$ $\left(162 \mathrm{MHz}, \mathrm{CDCl}_{3}\right) \delta 34.41$; HRMS (ESI) calcd. for $\mathrm{C}_{13} \mathrm{H}_{19} \mathrm{NO}_{2} \mathrm{P}[\mathrm{M}+\mathrm{H}]^{+}: 252.1153$, found 252.1154 .

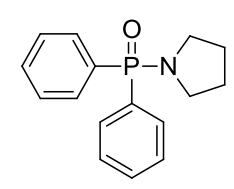

$3 n$

3n (44 mg, 82\%, reaction time: $8 \mathrm{~h})\left(\mathrm{R}_{\mathrm{f}} 0.4\right.$, EA) was prepared following the typical procedure in $0.20 \mathrm{mmol}$ scale as yellow oil. ${ }^{1} \mathbf{H}$ NMR $\left(400 \mathrm{MHz}, \mathrm{CDCl}_{3}\right) \delta 8.06-7.87(\mathrm{~m}, 2$ H), 7.87-7.73 (m, $2 \mathrm{H}), 7.58-7.37(\mathrm{~m}, 6 \mathrm{H}), 3.87(\mathrm{td}, J=8.4,3.6 \mathrm{~Hz}, 1 \mathrm{H}), 3.09-2.96(\mathrm{~m}, 1 \mathrm{H})$, 2.96-2.83 (m, $1 \mathrm{H}), 2.17$ (bs, $1 \mathrm{H}), 2.08-1.87(\mathrm{~m}, 2 \mathrm{H}), 1.87-1.62(\mathrm{~m}, 2 \mathrm{H}) ;{ }^{13} \mathbf{C}$ NMR $(100$ $\left.\mathrm{MHz}, \mathrm{CDCl}_{3}\right) \delta 133.0,131.8(\mathrm{~d}, J=8.6 \mathrm{~Hz}), 131.5(\mathrm{~d}, J=112.6 \mathrm{~Hz}), 131.7(\mathrm{dd}, J=12.0,2.7$ $\mathrm{Hz}), 131.1$ (d, $J=8.7 \mathrm{~Hz}), 128.5$ (dd, $J=11.2,4.2 \mathrm{~Hz}), 56.9$ (d, $J=84.4 \mathrm{~Hz}), 48.3$ (d, $J=8.6$ $\mathrm{Hz}), 26.6,26.4(\mathrm{~d}, J=6.5 \mathrm{~Hz}) ;{ }^{31} \mathbf{P}$ NMR $\left(162 \mathrm{MHz}, \mathrm{CDCl}_{3}\right) \delta 31.43$; HRMS (ESI) calcd. for $\mathrm{C}_{16} \mathrm{H}_{19} \mathrm{NOP}[\mathrm{M}+\mathrm{H}]^{+}:$272.1204, found 272.1208.

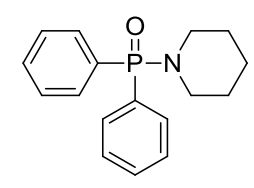

30

3o $(52 \mathrm{mg}$, 91\%, reaction time: $8 \mathrm{~h})\left(\mathrm{R}_{\mathrm{f}} 0.4\right.$, EA) was prepared following the typical procedure in $0.20 \mathrm{mmol}$ scale as white solid. ${ }^{1} \mathbf{H}$ NMR $\left(400 \mathrm{MHz}, \mathrm{CDCl}_{3}\right) \delta$ 7.94-7.79 (m, 4 $\mathrm{H})$, 7.54-7.35 (m, $6 \mathrm{H}), 3.00(\mathrm{dd}, J=10.8,6.4 \mathrm{~Hz}, 4 \mathrm{H}), 1.72-1.45(\mathrm{~m}, 6 \mathrm{H}) ;{ }^{13} \mathbf{C}$ NMR $(100$ $\left.\mathrm{MHz}, \mathrm{CDCl}_{3}\right) \delta 132.2(\mathrm{~d}, J=10.0 \mathrm{~Hz}), 132.0(\mathrm{~d}, J=128.5 \mathrm{~Hz}), 131.5(\mathrm{~d}, J=2.7 \mathrm{~Hz}), 128.5$ $(\mathrm{d}, J=12.3 \mathrm{~Hz}), 45.7,26.2(\mathrm{~d}, J=6.8 \mathrm{~Hz}), 24.5 ;{ }^{31} \mathbf{P} \mathbf{N M R}\left(162 \mathrm{MHz}, \mathrm{CDCl}_{3}\right) \delta 28.98$; 
HRMS (ESI) calcd. for $\mathrm{C}_{17} \mathrm{H}_{21} \mathrm{NOP}[\mathrm{M}+\mathrm{H}]^{+}:$286.1361, found 286.1357.

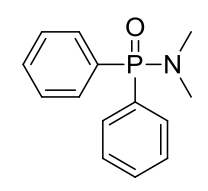

$3 p$

3p (45 mg, 91\%, reaction time: $9 \mathrm{~h})\left(\mathrm{R}_{\mathrm{f}} 0.3\right.$, EA) was prepared following the typical procedure in $0.20 \mathrm{mmol}$ scale as colorless oil. ${ }^{1} \mathbf{H}$ NMR $\left(400 \mathrm{MHz}, \mathrm{CDCl}_{3}\right) \delta$ 7.93-7.76 (m, 4 $\mathrm{H}), 7.55-7.36$ (m, $6 \mathrm{H}), 2.66(\mathrm{~s}, 3 \mathrm{H}), 2.63(\mathrm{~s}, 3 \mathrm{H}) ;{ }^{13} \mathbf{C ~ N M R}\left(100 \mathrm{MHz}, \mathrm{CDCl}_{3}\right) \delta 132.3(\mathrm{~d}$, $J=9.2 \mathrm{~Hz}), 131.8(\mathrm{~d}, J=128.4 \mathrm{~Hz}) 131.7(\mathrm{~d}, J=2.7 \mathrm{~Hz}), 128.5(\mathrm{~d}, J=12.3 \mathrm{~Hz}), 37.1(\mathrm{~d}, J=$ $2.4 \mathrm{~Hz}) ;{ }^{31} \mathbf{P}$ NMR $\left(162 \mathrm{MHz}, \mathrm{CDCl}_{3}\right) \delta 31.27$; HRMS (ESI) calcd. for $\mathrm{C}_{14} \mathrm{H}_{17} \mathrm{NOP}[\mathrm{M}+\mathrm{H}]^{+}$: 246.1048 , found 246.1044 .

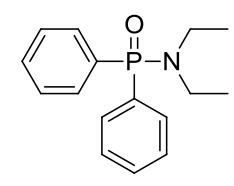

$3 q$

3q (37 mg, 67\%, reaction time: $10 \mathrm{~h})\left(\mathrm{R}_{\mathrm{f}} 0.3\right.$, EA) was prepared following the typical procedure in $0.20 \mathrm{mmol}$ scale as yellow oil. ${ }^{1} \mathbf{H}$ NMR $\left(400 \mathrm{MHz}, \mathrm{CDCl}_{3}\right) \delta 8.00-7.80(\mathrm{~m}, 4$ H), 7.60-7.37 (m, $6 \mathrm{H}), 3.61-3.47(\mathrm{~m}, 1 \mathrm{H}), 2.89-2.72(\mathrm{~m}, 1 \mathrm{H}), 2.58-2.40(\mathrm{~m}, 1 \mathrm{H}), 1.85$ (bs, $1 \mathrm{H}), 1.31(\mathrm{dd}, J=15.6,6.8 \mathrm{~Hz}, 3 \mathrm{H}), 1.01(\mathrm{t}, J=7.2 \mathrm{~Hz}, 3 \mathrm{H}) ;{ }^{13} \mathbf{C ~ N M R}\left(100 \mathrm{MHz}, \mathrm{CDCl}_{3}\right)$ $\delta 131.8(\mathrm{~d}, J=183.7 \mathrm{~Hz}), 131.68(\mathrm{dd}, J=40.6,8.4 \mathrm{~Hz}), 131.67(\mathrm{t}, J=2.5 \mathrm{~Hz}), 128.4(\mathrm{dd}, J=$ 15.6, $11.1 \mathrm{~Hz}), 52.6(\mathrm{~d}, J=83.6 \mathrm{~Hz}), 42.3(\mathrm{~d}, J=11.7 \mathrm{~Hz}), 15.3,14.2(\mathrm{~d}, J=1.8 \mathrm{~Hz}) ;{ }^{31} \mathbf{P}$ NMR $\left(162 \mathrm{MHz}, \mathrm{CDCl}_{3}\right) \delta 32.11$; HRMS (ESI) calcd. for $\mathrm{C}_{16} \mathrm{H}_{21} \mathrm{NOP}[\mathrm{M}+\mathrm{H}]^{+}:$274.1361, found 274.1361 .

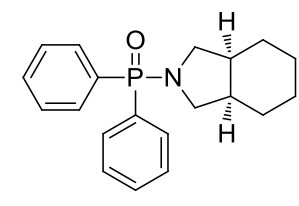

3s

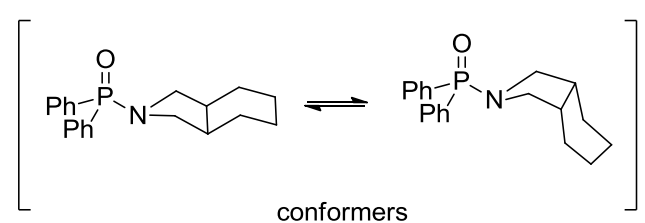

conformers

3s (56 mg, 86\%, reaction time: $12 \mathrm{~h})\left(\mathrm{R}_{\mathrm{f}} 0.3\right.$, EA) was prepared following the typical procedure in $0.20 \mathrm{mmol}$ scale as white solid. Conformers were observed by NMR analysis. ${ }^{1} \mathbf{H}$ NMR $\left(400 \mathrm{MHz}, \mathrm{CDCl}_{3}\right) \delta$ 8.11-7.92 (m, $\left.2 \mathrm{H}\right)$, 7.92-7.75 (m, $\left.2 \mathrm{H}\right)$, 7.62-7.34 (m, $\left.6 \mathrm{H}\right)$, $4.20(\mathrm{t}, J=8.8 \mathrm{~Hz}, 0.1 \mathrm{H}), 3.80(\mathrm{dd}, J=6.8,4.4 \mathrm{~Hz}, 0.9 \mathrm{H}), 3.22-3.12(\mathrm{~m}, 0.1 \mathrm{H}), 2.89-2.68$ $(\mathrm{m}, 1.9 \mathrm{H}), 2.56-2.34(\mathrm{~m}, 1 \mathrm{H}), 2.15(\mathrm{~s}, 1 \mathrm{H}), 2.08-1.92(\mathrm{~m}, 1 \mathrm{H}), 1.69-1.11(\mathrm{~m}, 8 \mathrm{H}) ;{ }^{13} \mathrm{C}$ NMR $\left(100 \mathrm{MHz}, \mathrm{CDCl}_{3}\right) \delta 133.1(\mathrm{~d}, J=92.8 \mathrm{~Hz}), 131.9,131.7(\mathrm{dd}, J=99.4,8.4 \mathrm{~Hz}), 131.6$ (dd, $J=12.4,2.6 \mathrm{~Hz}), 130.9,128.4(\mathrm{dd}, J=11.0,8.8 \mathrm{~Hz}), 59.9,59.0,51.5(\mathrm{~d}, J=4.7 \mathrm{~Hz})$, $39.0(\mathrm{~d}, J=3.3 \mathrm{~Hz}), 27.3(\mathrm{~d}, J=5.3 \mathrm{~Hz}), 26.0,23.4,22.6 ;{ }^{31} \mathbf{P} \mathbf{N M R}\left(162 \mathrm{MHz}, \mathrm{CDCl}_{3}\right) \delta$ 30.63; HRMS (ESI) calcd. for $\mathrm{C}_{20} \mathrm{H}_{25} \mathrm{NOP}[\mathrm{M}+\mathrm{H}]^{+}: 326.1674$, found 326.1677. 


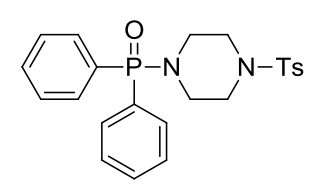

$3 \mathbf{t}$

$3 t$ (42 mg, 48\%, reaction time: $12 \mathrm{~h})\left(\mathrm{R}_{\mathrm{f}} 0.3\right.$, EA) was prepared following the typical procedure in $0.20 \mathrm{mmol}$ scale as yellow solid. ${ }^{1} \mathbf{H}$ NMR $\left(400 \mathrm{MHz}, \mathrm{CDCl}_{3}\right) \delta$ 7.93-7.70 (m, $4 \mathrm{H}), 7.65-7.4(\mathrm{~m}, 7 \mathrm{H}), 7.28(\mathrm{~d}, J=8.0 \mathrm{~Hz}, 3 \mathrm{H}), 3.81-3.68(\mathrm{~m}, 1 \mathrm{H}), 3.64(\mathrm{~d}, J=12.0 \mathrm{~Hz}, 2 \mathrm{H})$, 3.51 (bs, $1 \mathrm{H}), 3.09$ (d, $J=13.2 \mathrm{~Hz}, 1 \mathrm{H}), 2.99-2.85(\mathrm{~m}, 1 \mathrm{H}), 2.52-2.35(\mathrm{~m}, 4 \mathrm{H}), 2.32-2.18$ $(\mathrm{m}, 1 \mathrm{H}) ;{ }^{13} \mathbf{C}$ NMR $\left(100 \mathrm{MHz}, \mathrm{CDCl}_{3}\right) \delta 143.9,132.5(\mathrm{dd}, J=21.8,2.7 \mathrm{~Hz}), 132.3,131.4(\mathrm{~d}$, $J=11.1 \mathrm{~Hz}), 131.2(\mathrm{~d}, J=9.1 \mathrm{~Hz}), 129.0(\mathrm{~d}, J=10.6 \mathrm{~Hz}), 128.8(\mathrm{~d}, J=11.7 \mathrm{~Hz}), 128.7(\mathrm{~d}, J$ $=209.5 \mathrm{~Hz}), 53.8(\mathrm{~d}, J=80.4 \mathrm{~Hz}), 46.4,45.6(\mathrm{dd}, J=34.5,6.3 \mathrm{~Hz}), 29.7,21.5 ;{ }^{31} \mathbf{P} \mathbf{~ N M R}$ $\left(162 \mathrm{MHz}, \mathrm{CDCl}_{3}\right) \delta$ 28.88; HRMS (ESI) calcd. for $\mathrm{C}_{23} \mathrm{H}_{26} \mathrm{~N}_{2} \mathrm{O}_{3} \mathrm{PS}[\mathrm{M}+\mathrm{H}]^{+}: 441.1402$, found 441.1401 .

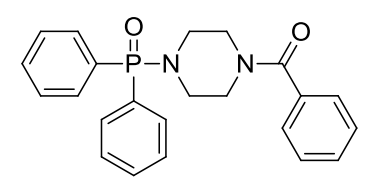

$3 \mathbf{u}$

3u (64 mg, 82\%, reaction time: $8 \mathrm{~h})\left(\mathrm{R}_{\mathrm{f}} 0.3\right.$, EA) was prepared following the typical procedure in $0.20 \mathrm{mmol}$ scale as yellow oil. ${ }^{1} \mathbf{H}$ NMR $\left(400 \mathrm{MHz}, \mathrm{CDCl}_{3}\right) \delta 7.93-7.76(\mathrm{~m}, 4$ H), 7.60-7.41 (m, $6 \mathrm{H}), 7.41-7.28(\mathrm{~m}, 5 \mathrm{H}), 3.61$ (bd, $J=12.8 \mathrm{~Hz}, 4 \mathrm{H}), 3.08(\mathrm{bs}, 4 \mathrm{H}) ;{ }^{13} \mathbf{C}$ NMR $\left(100 \mathrm{MHz}, \mathrm{CDCl}_{3}\right) \delta 170.5,135.4,132.2(\mathrm{~d}, J=9.2 \mathrm{~Hz}), 132.1(\mathrm{~d}, J=2.7 \mathrm{~Hz}), 130.7(\mathrm{~d}$, $J=128.1 \mathrm{~Hz}), 129.8,128.7(\mathrm{~d}, J=12.4 \mathrm{~Hz}), 128.5,126.9,45.0(\mathrm{~m}) ;{ }^{31} \mathbf{P}$ NMR $(162 \mathrm{MHz}$, $\left.\mathrm{CDCl}_{3}\right) \delta$ 29.66; HRMS (ESI) calcd. for $\mathrm{C}_{23} \mathrm{H}_{24} \mathrm{~N}_{2} \mathrm{O}_{2} \mathrm{P}[\mathrm{M}+\mathrm{H}]^{+}: 391.1575$, found 391.1576.

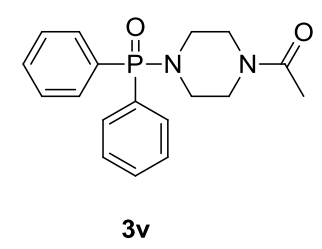

3v (49 mg, 74\%, reaction time: $10 \mathrm{~h})\left(\mathrm{R}_{\mathrm{f}} 0.3\right.$, EA) was prepared following the typical procedure in $0.20 \mathrm{mmol}$ scale as yellow oil. ${ }^{1} \mathbf{H}$ NMR $\left(400 \mathrm{MHz}, \mathrm{CDCl}_{3}\right) \delta$ 7.92-7.77 (m, 4 H), 7.58-7.40 (m, $6 \mathrm{H}), 3.68-3.56$ (m, $2 \mathrm{H}), 3.53-3.39$ (m, $2 \mathrm{H}), 3.16-3.06$ (m, $2 \mathrm{H}), 3.06-2.97$ $(\mathrm{m}, 2 \mathrm{H}), 2.06(\mathrm{~s}, 3 \mathrm{H}) ;{ }^{13} \mathbf{C ~ N M R}\left(75 \mathrm{MHz}, \mathrm{CDCl}_{3}\right) \delta 169.1,132.2(\mathrm{~d}, J=9.2 \mathrm{~Hz}), 132.1(\mathrm{~d}, J$ $=2.7 \mathrm{~Hz}), 130.8(\mathrm{~d}, J=170.8 \mathrm{~Hz}), 128.8(\mathrm{~d}, J=12.5 \mathrm{~Hz}), 46.9(\mathrm{~d}, J=6.3 \mathrm{~Hz}), 44.9$ (d, $J=$ $5.9 \mathrm{~Hz}), 41.9(\mathrm{~d}, J=6.1 \mathrm{~Hz}), 21.3 ;{ }^{31} \mathbf{P}$ NMR $\left(162 \mathrm{MHz}, \mathrm{CDCl}_{3}\right) \delta 29.70$; HRMS (ESI) calcd. for $\mathrm{C}_{18} \mathrm{H}_{22} \mathrm{~N}_{2} \mathrm{O}_{2} \mathrm{P}[\mathrm{M}+\mathrm{H}]^{+}:$329.1419, found 329.1414.

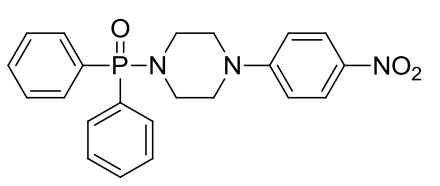

$3 w$ 
3w (52 mg, 64\%, reaction time: $10 \mathrm{~h})\left(\mathrm{R}_{\mathrm{f}} 0.2\right.$, EA) was prepared following the typical procedure in $0.20 \mathrm{mmol}$ scale as yellow solid. ${ }^{1} \mathbf{H}$ NMR $\left(400 \mathrm{MHz}, \mathrm{CDCl}_{3}\right) \delta 8.18-8.07(\mathrm{~m}, 2$ H), 7.97-7.84 (m, 4 H), 7.62-7.44 (m, 6 H), 6.87-6.75 (m, 2 H), 3.47-3.38 (m, 4 H), 3.31-3.20 $(\mathrm{m}, 4 \mathrm{H}) ;{ }^{13} \mathbf{C ~ N M R}\left(100 \mathrm{MHz}, \mathrm{CDCl}_{3}\right) \delta 154.9,139.0,132.3(\mathrm{~d}, J=9.2 \mathrm{~Hz}), 132.2(\mathrm{~d}, J=2.7$ $\mathrm{Hz}), 130.8(\mathrm{~d}, J=128.3 \mathrm{~Hz}), 128.8(\mathrm{~d}, J=12.5 \mathrm{~Hz}), 125.9,113.10,47.8(\mathrm{~d}, J=6.5 \mathrm{~Hz}), 44.5$; ${ }^{31} \mathbf{P}$ NMR $\left(162 \mathrm{MHz}, \mathrm{CDCl}_{3}\right) \delta$ 29.53; HRMS (ESI) calcd. for $\mathrm{C}_{22} \mathrm{H}_{23} \mathrm{~N}_{3} \mathrm{O}_{3} \mathrm{P}[\mathrm{M}+\mathrm{H}]^{+}$: 408.1477, found 408.1477 .

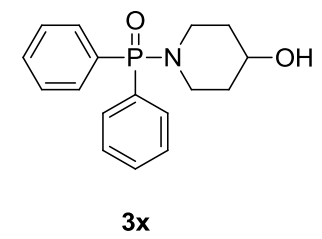

3x (40 mg, 67\%, reaction time: $8 \mathrm{~h})\left(\mathrm{R}_{\mathrm{f}} 0.1\right.$, EA) was prepared following the typical procedure in $0.20 \mathrm{mmol}$ scale as yellow oil. ${ }^{1} \mathbf{H}$ NMR $\left(400 \mathrm{MHz}, \mathrm{CDCl}_{3}\right) \delta$ 7.98-7.75 (m, 4 H), 7.58-7.35 (m, $6 \mathrm{H}), 3.93-3.72(\mathrm{~m}, 1 \mathrm{H}), 3.42-3.18(\mathrm{~m}, 2 \mathrm{H}), 2.96-2.74(\mathrm{~m}, 2 \mathrm{H}), 2.54$ (bs, $1 \mathrm{H}), 1.97-1.79(\mathrm{~m}, 2 \mathrm{H}), 1.66-1.46(\mathrm{~m}, 2 \mathrm{H}) ;{ }^{13} \mathbf{C ~ N M R}\left(100 \mathrm{MHz}, \mathrm{CDCl}_{3}\right) \delta 132.2(\mathrm{~d}, J=$ $9.2 \mathrm{~Hz}), 131.8(\mathrm{~d}, J=2.7 \mathrm{~Hz}), 131.6(\mathrm{~d}, J=128.5 \mathrm{~Hz}), 128.6(\mathrm{~d}, J=12.4 \mathrm{~Hz}), 67.7,42.8$, $34.9(\mathrm{~d}, J=6.5 \mathrm{~Hz}) ;{ }^{31} \mathbf{P}$ NMR $\left(162 \mathrm{MHz}, \mathrm{CDCl}_{3}\right) \delta 29.52$; HRMS (ESI) calcd. for $\mathrm{C}_{17} \mathrm{H}_{21} \mathrm{NO}_{2} \mathrm{P}[\mathrm{M}+\mathrm{H}]^{+}:$302.1310, found 302.1310.

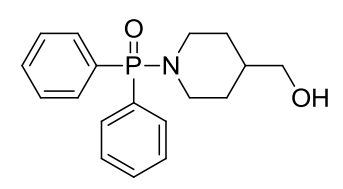

$3 y$

3y (56 mg, 89\%, reaction time: $10 \mathrm{~h})\left(\mathrm{R}_{\mathrm{f}} 0.2\right.$, EA) was prepared following the typical procedure in $0.20 \mathrm{mmol}$ scale as yellow oil. ${ }^{1} \mathbf{H}$ NMR $\left(400 \mathrm{MHz}, \mathrm{CDCl}_{3}\right) \delta$ 7.98-7.67 (m, 4 H), 7.57-7.34 (m, $6 \mathrm{H}), 3.50(\mathrm{~d}, J=5.6 \mathrm{~Hz}, 2 \mathrm{H}), 3.37-3.20(\mathrm{~m}, 2 \mathrm{H}), 2.91(\mathrm{bs}, 1 \mathrm{H}), 2.84-2.68$ (m, $2 \mathrm{H}), 1.76-1.57$ (m, $2 \mathrm{H}), 1.37-1.15$ (m, $2 \mathrm{H}) ;{ }^{13} \mathbf{C ~ N M R}\left(100 \mathrm{MHz}, \mathrm{CDCl}_{3}\right) \delta 132.2(\mathrm{~d}, J$ $=9.2 \mathrm{~Hz}), 131.7(\mathrm{~d}, J=2.7 \mathrm{~Hz}), 130.9,128.5(\mathrm{~d}, J=12.3 \mathrm{~Hz}), 67.3,44.9,38.7,29.2(\mathrm{~d}, J=$ $6.7 \mathrm{~Hz}) ;{ }^{31} \mathbf{P}$ NMR $\left(162 \mathrm{MHz}, \mathrm{CDCl}_{3}\right) \delta 29.50$; HRMS (ESI) calcd. for $\mathrm{C}_{18} \mathrm{H}_{23} \mathrm{NO}_{2} \mathrm{P}[\mathrm{M}+\mathrm{H}]^{+}$: 316.1466 , found 316.1468 .

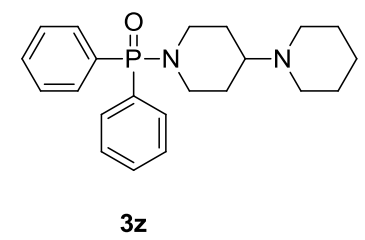

3z (39 mg, 53\%, reaction time: $8 \mathrm{~h})\left(\mathrm{R}_{\mathrm{f}} 0.5\right.$, dichloromethane/methanol $20 / 1+2 \%$ triethylamine) was prepared following the typical procedure in $0.20 \mathrm{mmol}$ scale as yellow solid. ${ }^{1} \mathbf{H}$ NMR (400 MHz, $\left.\mathrm{CDCl}_{3}\right) \delta$ 8.08-7.74 (m, $\left.4 \mathrm{H}\right)$, 7.58-7.32 (m, $\left.6 \mathrm{H}\right), 3.40-3.24$ (m, 2 H), 2.83-2.66 (m, $2 \mathrm{H}), 2.48(\mathrm{t}, J=5.0 \mathrm{~Hz}, 4 \mathrm{H}), 2.42-2.26(\mathrm{~m}, 1 \mathrm{H}), 1.77(\mathrm{bd}, J=12.0 \mathrm{~Hz}, 2$ $\mathrm{H}), 1.65-1.45(\mathrm{~m}, 6 \mathrm{H}), 1.45-1.31(\mathrm{~m}, 2 \mathrm{H}) ;{ }^{13} \mathbf{C ~ N M R}\left(100 \mathrm{MHz}, \mathrm{CDCl}_{3}\right) \delta 132.1(\mathrm{~d}, J=9.0$ $\mathrm{Hz}), 131.7$ (d, $J=128.6 \mathrm{~Hz}), 131.6(\mathrm{~d}, J=2.7 \mathrm{~Hz}), 128.46$ (d, $J=12.3 \mathrm{~Hz}), 62.4,50.1,44.7$, $28.7(\mathrm{~d}, J=7.3 \mathrm{~Hz}), 26.2,24.6 ;{ }^{31} \mathbf{P}$ NMR $\left(162 \mathrm{MHz}, \mathrm{CDCl}_{3}\right) \delta 28.92$; HRMS (ESI) calcd. for $\mathrm{C}_{22} \mathrm{H}_{30} \mathrm{~N}_{2} \mathrm{OP}[\mathrm{M}+\mathrm{H}]^{+}:$369.2096, found 369.2091. 


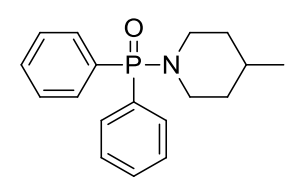

3A

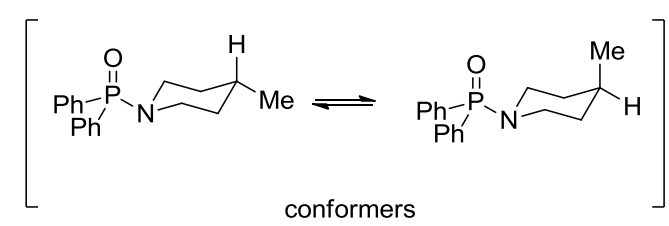

conformers

3A (40 mg, 67\%, reaction time: $10 \mathrm{~h})\left(\mathrm{R}_{\mathrm{f}} 0.2\right.$, EA) was prepared following the typical procedure in $0.20 \mathrm{mmol}$ scale as yellow oil. Conformers were observed by NMR analysis. ${ }^{1}$ H NMR $\left(400 \mathrm{MHz}, \mathrm{CDCl}_{3}\right) \delta$ 8.00-7.72 (m, $\left.4 \mathrm{H}\right)$, 7.60-7.37 (m, $\left.6 \mathrm{H}\right), 3.77-3.67(\mathrm{~m}, 0.7 \mathrm{H})$, 3.49-3.40 (m, 0.3 H), 3.17-3.09 (m, 0.3 H), 3.07-2.96 (m, 0.7 H), 2.88-2.77 (m, 0.7 H), 2.60 (td, $J=12.4,2.4 \mathrm{~Hz}, 0.3 \mathrm{H}), 2.25-2.09(\mathrm{~m}, 1 \mathrm{H}), 1.90-1.79(\mathrm{~m}, 1 \mathrm{H}), 1.79-1.41(\mathrm{~m}, 3 \mathrm{H})$, 1.34-1.23 (m, $1 \mathrm{H}), 0.99$ (d, $J=6.8 \mathrm{~Hz}, 2 \mathrm{H}), 0.87(\mathrm{~d}, J=6.4 \mathrm{~Hz}, 1 \mathrm{H}) ;{ }^{13} \mathbf{C}$ NMR $(100 \mathrm{MHz}$, $\left.\mathrm{CDCl}_{3}\right) \delta 131.9(\mathrm{~d}, J=3.2 \mathrm{~Hz}), 131.80(\mathrm{~d}, J=91.8 \mathrm{~Hz}), 131.79(\mathrm{~d}, J=1.8 \mathrm{~Hz}), 131.7(\mathrm{~d}, J=$ $2.7 \mathrm{~Hz}), 131.6(\mathrm{~d}, J=8.5 \mathrm{~Hz}), 131.4(\mathrm{~d}, J=8.8 \mathrm{~Hz}), 131.2(\mathrm{~d}, J=8.5 \mathrm{~Hz}), 128.6(\mathrm{~d}, J=2.4$ $\mathrm{Hz}), 128.5$ (d, $J=1.7 \mathrm{~Hz}), 128.4,56.8,56.0,51.5,50.7,47.5$ (d, $J=14.3 \mathrm{~Hz}), 41.9$ (d, $J=$ $10.0 \mathrm{~Hz}), 34.9,33.5,32.4,31.6(\mathrm{~d}, J=12.5 \mathrm{~Hz}), 31.5,25.8(\mathrm{~d}, J=8.5 \mathrm{~Hz}), 22.5,18.7 ;{ }^{31} \mathbf{P}$ NMR $\left(162 \mathrm{MHz}, \mathrm{CDCl}_{3}\right) \delta 32.73,31.19$; HRMS (ESI) calcd. for $\mathrm{C}_{18} \mathrm{H}_{23} \mathrm{NOP}[\mathrm{M}+\mathrm{H}]^{+}$: 300.1517 , found 300.1515 .

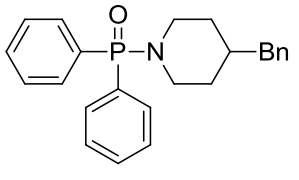

3B

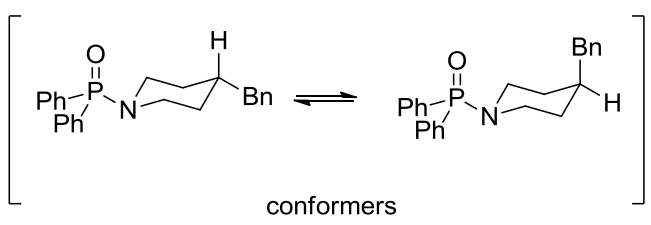

conformers

3B (41 mg, 55\%, reaction time: $7 \mathrm{~h})\left(\mathrm{R}_{\mathrm{f}} 0.2\right.$, EA) was prepared following the typical procedure in $0.20 \mathrm{mmol}$ scale as yellow oil. Conformers were observed by NMR analysis. ${ }^{1} \mathbf{H}$ NMR $\left(400 \mathrm{MHz}, \mathrm{CDCl}_{3}\right) \delta$ 8.05-7.83 (m, $\left.2 \mathrm{H}\right)$, 7.83-7.65 (m, $\left.2 \mathrm{H}\right)$, 7.59-7.45 (m, $\left.4 \mathrm{H}\right)$, 7.45-7.35 (m, $1 \mathrm{H})$, 7.33-7.00 (m, $6 \mathrm{H}), 3.78-3.70(\mathrm{~m}, 0.7 \mathrm{H}), 3.48-3.40(\mathrm{~m}, 0.3 \mathrm{H}), 3.16-3.04$ $(\mathrm{m}, 1 \mathrm{H}), 2.97-2.84(\mathrm{~m}, 1 \mathrm{H}), 2.76-2.59(\mathrm{~m}, 1 \mathrm{H}), 2.58-2.47(\mathrm{~m}, 1 \mathrm{H}), 2.44-2.26(\mathrm{~m}, 1 \mathrm{H})$, 1.87-1.74 (m, $2 \mathrm{H}), 1.74-1.61(\mathrm{~m}, 1 \mathrm{H}), 1.61-1.48(\mathrm{~m}, 1 \mathrm{H}), 1.44-1.25(\mathrm{~m}, 1 \mathrm{H}) ;{ }^{13} \mathbf{C}$ NMR $\left(100 \mathrm{MHz}, \mathrm{CDCl}_{3}\right) \delta 140.4,131.9(\mathrm{~d}, J=2.6 \mathrm{~Hz}), 131.7(\mathrm{~d}, J=2.9 \mathrm{~Hz}), 131.6(\mathrm{~d}, J=8.5 \mathrm{~Hz})$, $131.2(\mathrm{~d}, J=8.7 \mathrm{~Hz}), 129.0128 .59(\mathrm{dd}, J=12.3,11.4 \mathrm{~Hz}), 128.58(\mathrm{~d}, J=89.2 \mathrm{~Hz}), 128.2$, 51.9, 51.1, $42.2(\mathrm{~d}, J=9.4 \mathrm{~Hz}), 39.2,33.2(\mathrm{~d}, J=8.1 \mathrm{~Hz}), 30.4,29.3 ;{ }^{31} \mathbf{P}$ NMR $(162 \mathrm{MHz}$, $\left.\mathrm{CDCl}_{3}\right) \delta 32.88,31.36$; HRMS (ESI) calcd. for $\mathrm{C}_{24} \mathrm{H}_{27} \mathrm{NOP}[\mathrm{M}+\mathrm{H}]^{+}: 376.1830$, found 376.1832 . 
${ }^{1} \mathrm{H}$ NMR of $\mathbf{3 a}$
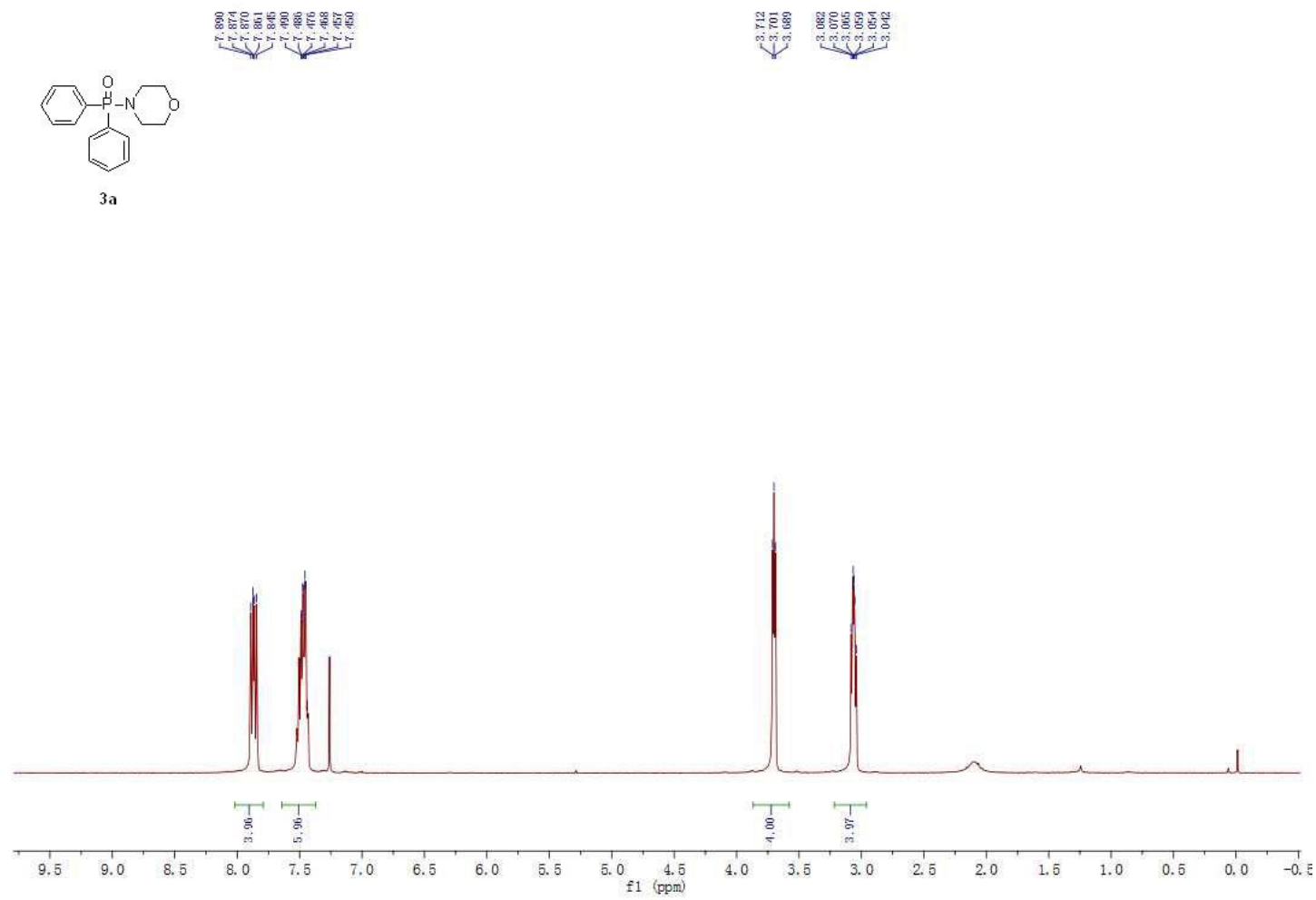

${ }^{13} \mathrm{C}$ NMR of $\mathbf{3 a}$
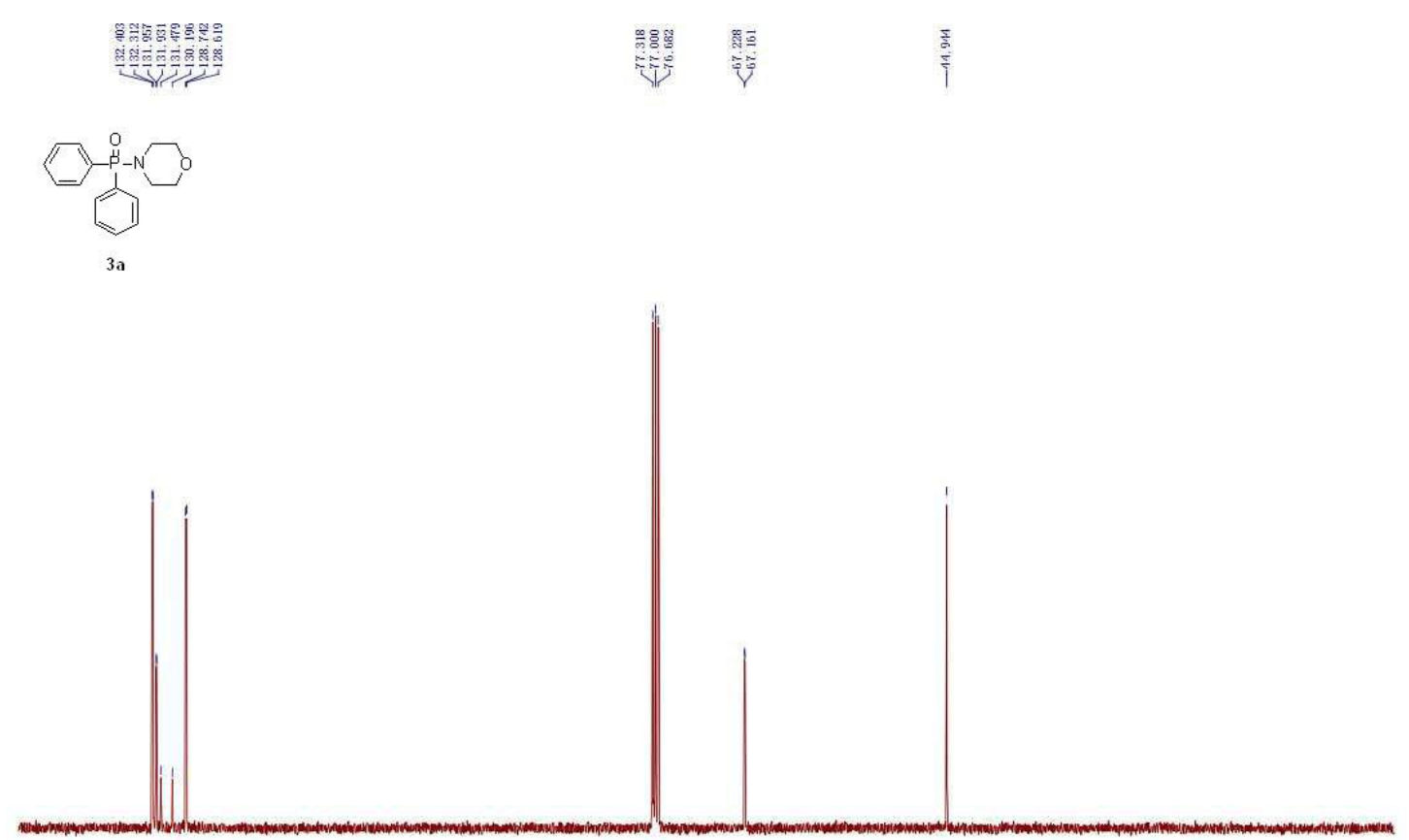

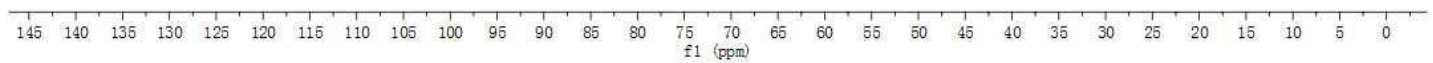


${ }^{31}$ P NMR of 3a

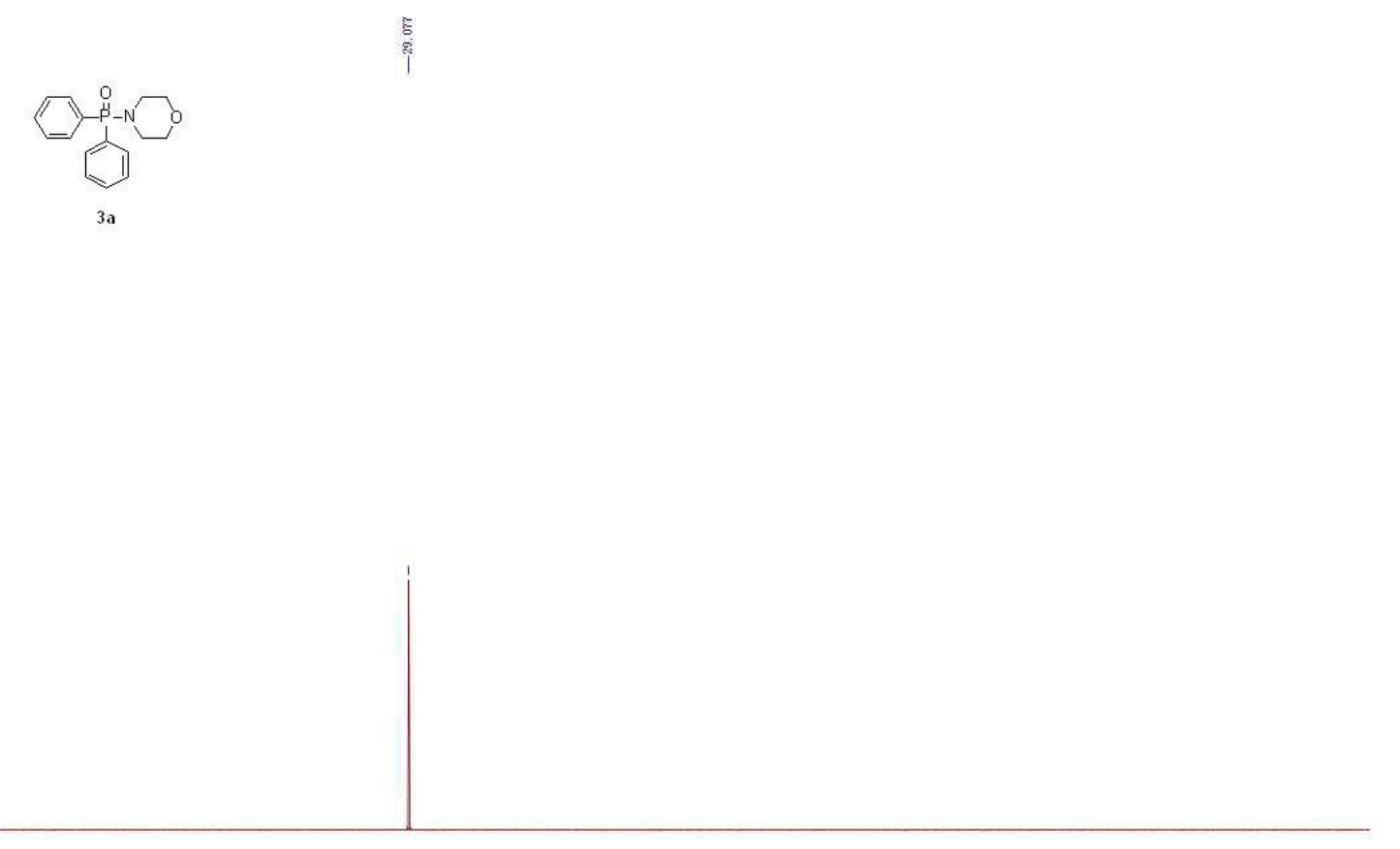

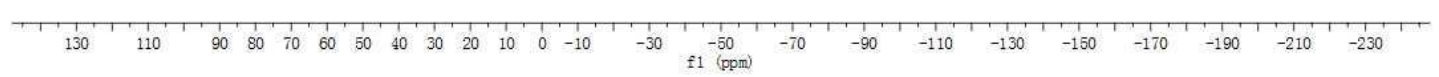

${ }^{1} \mathrm{H}$ NMR of $\mathbf{3 b}$
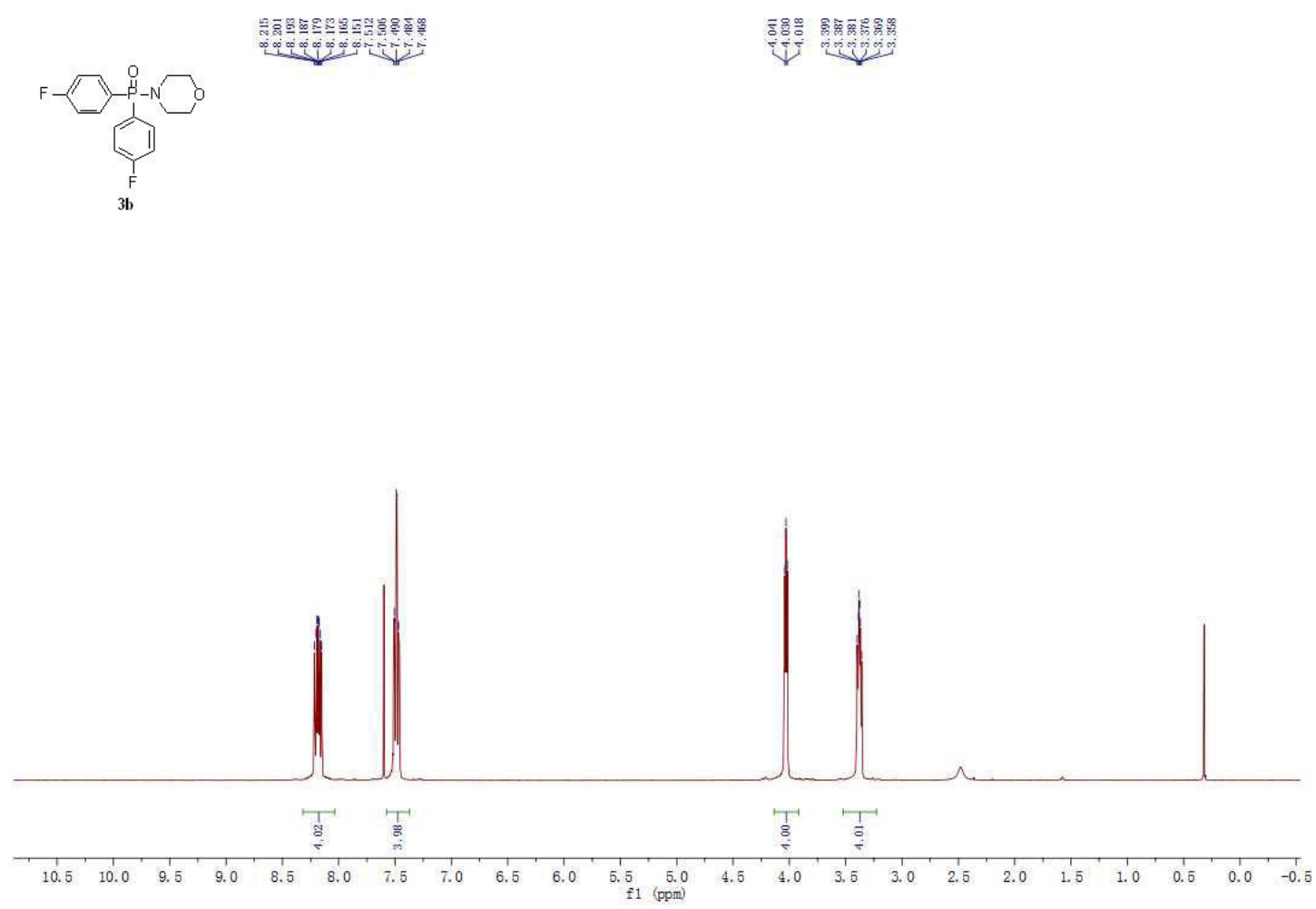
${ }^{13} \mathrm{C}$ NMR of $\mathbf{3 b}$
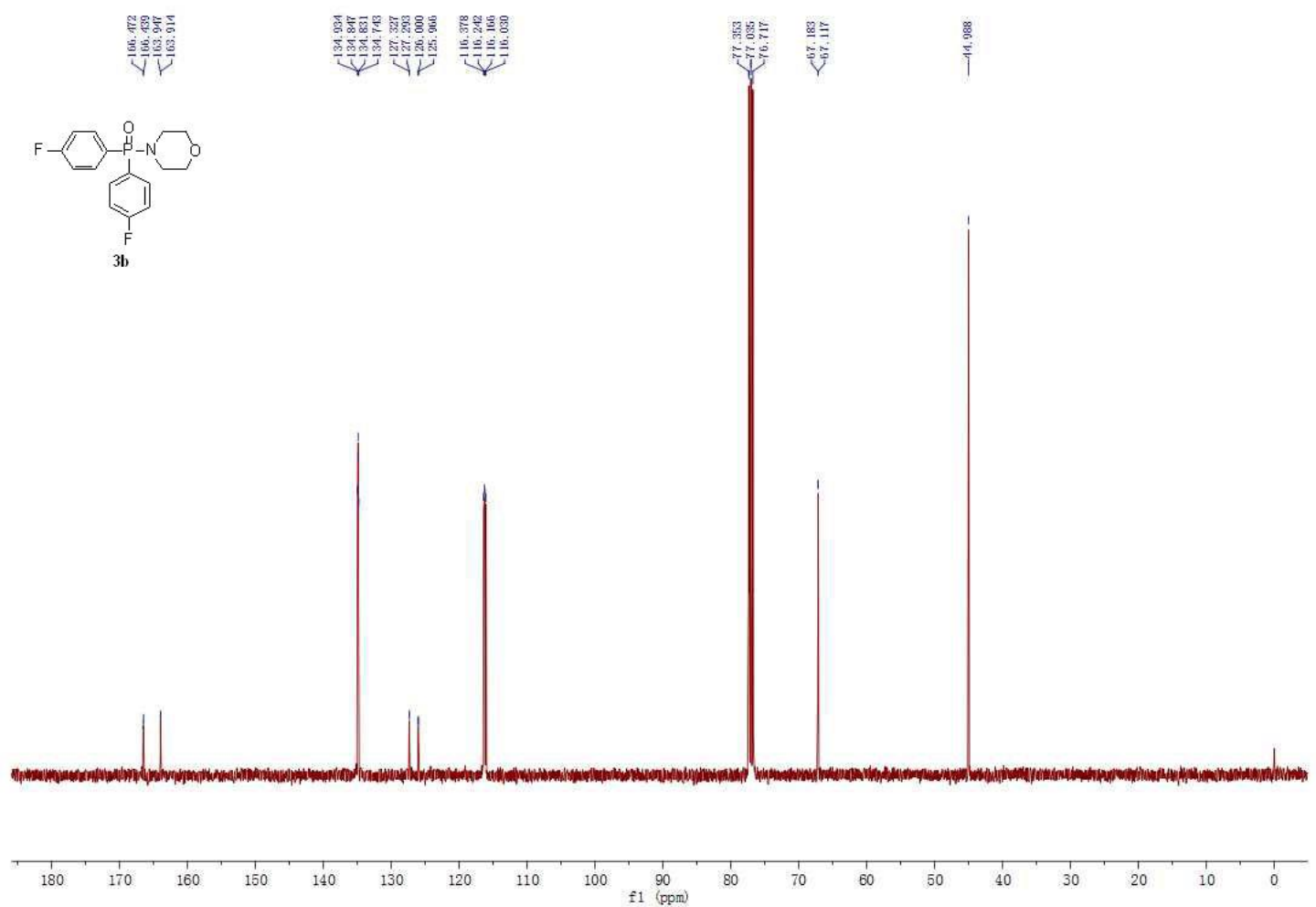

${ }^{19} \mathrm{~F}$ NMR of $\mathbf{3 b}$

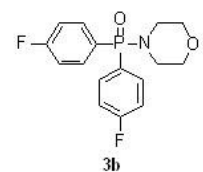

1

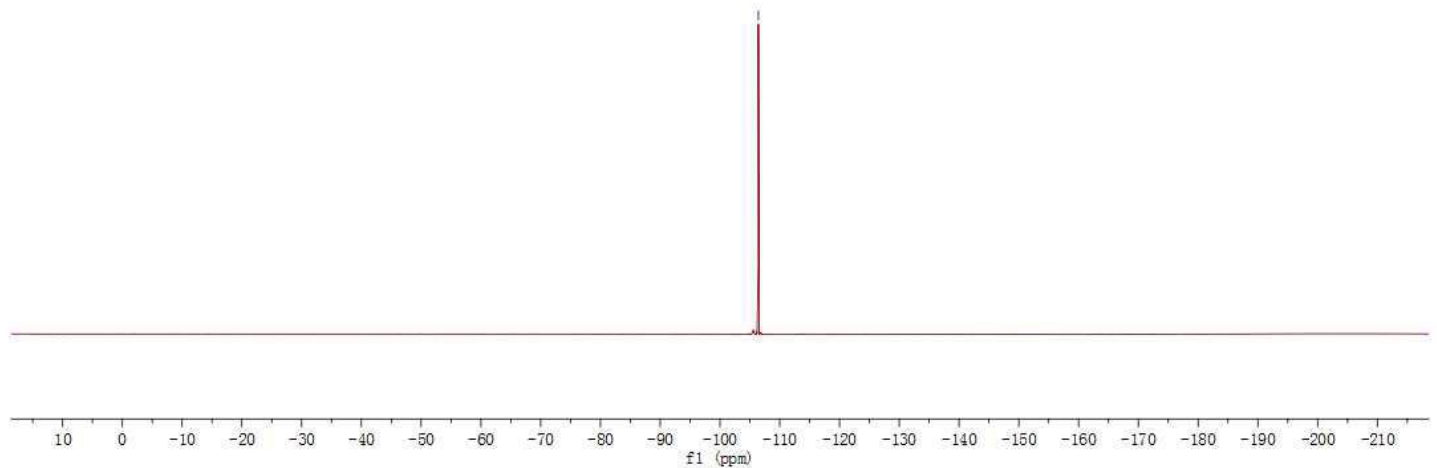


${ }^{31}$ P NMR of $\mathbf{3 b}$
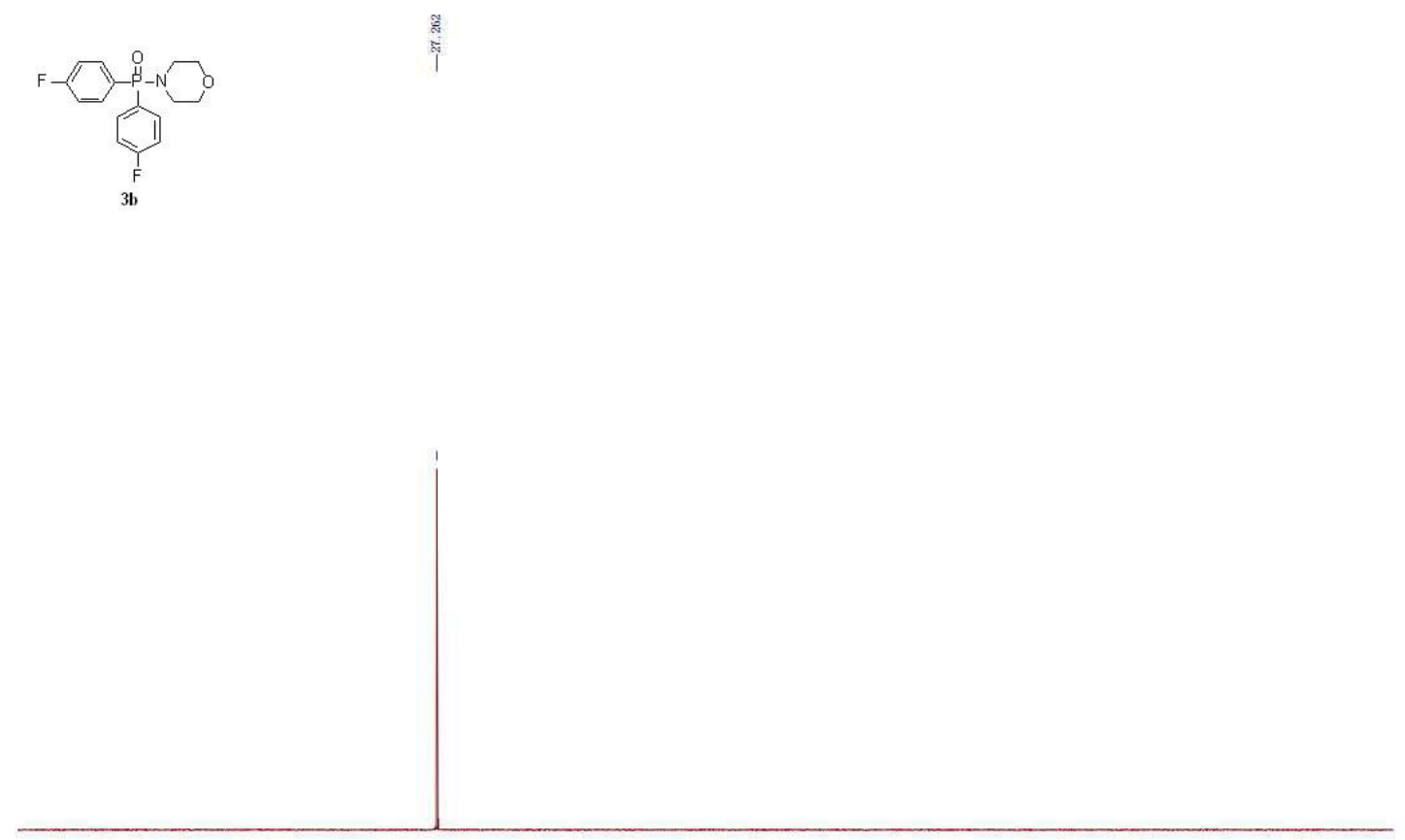

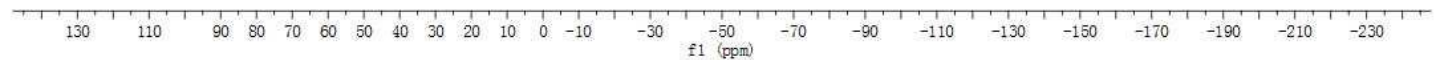
${ }^{1} \mathrm{H}$ NMR of $\mathbf{3 c}$

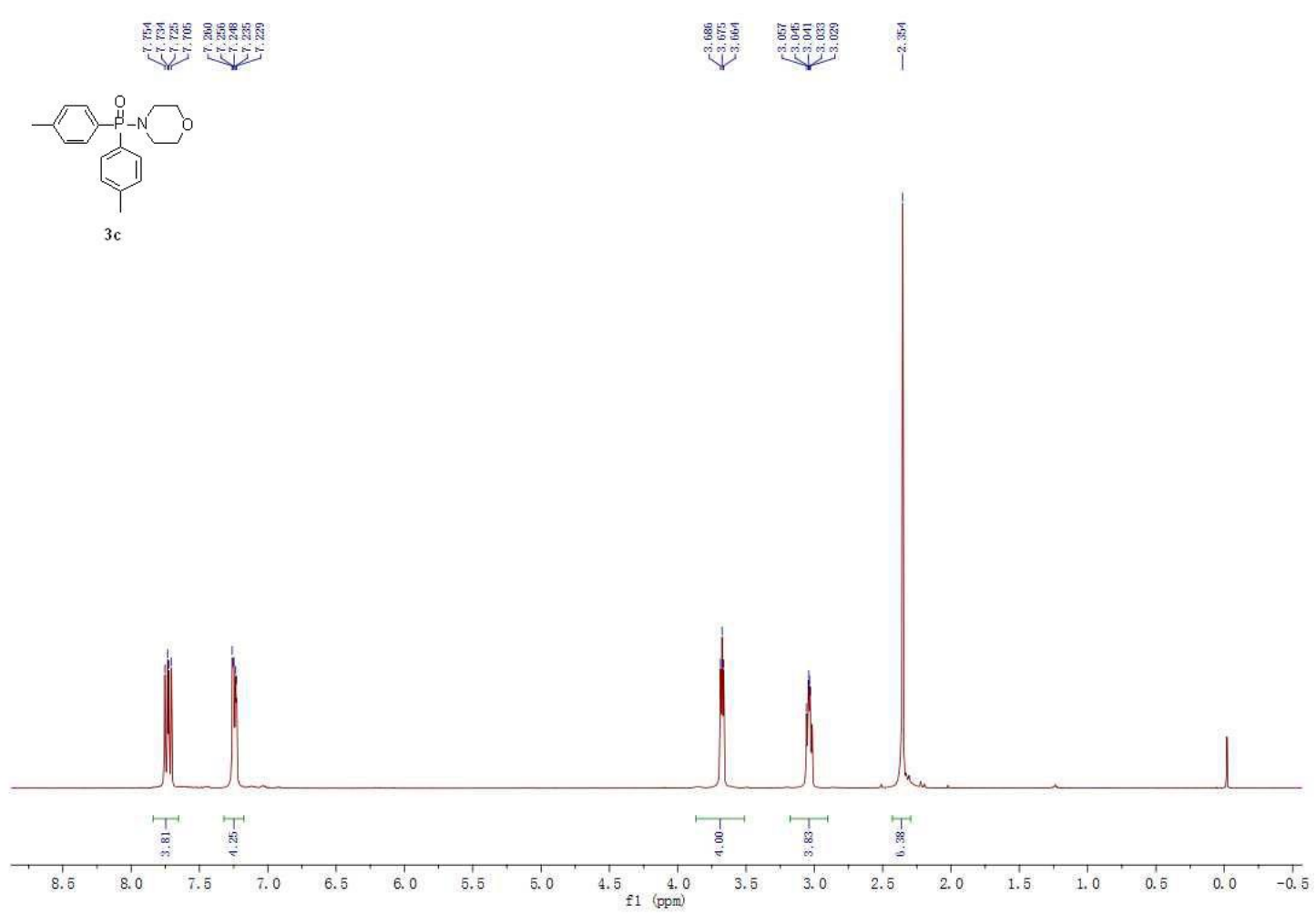


${ }^{13} \mathrm{C}$ NMR of $\mathbf{3 c}$

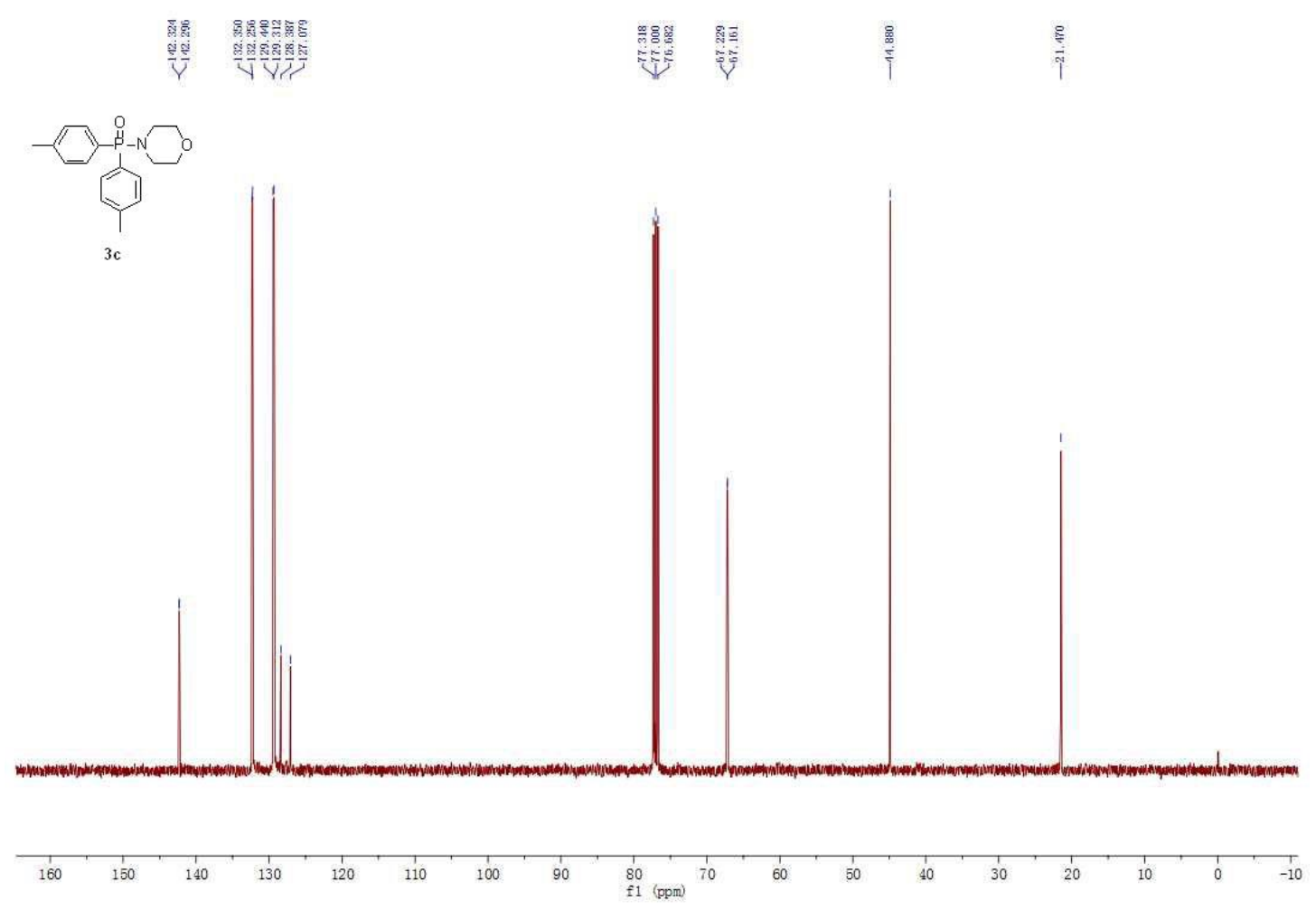

${ }^{31}$ P NMR of $\mathbf{3 c}$

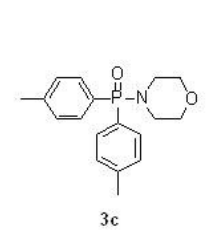

i

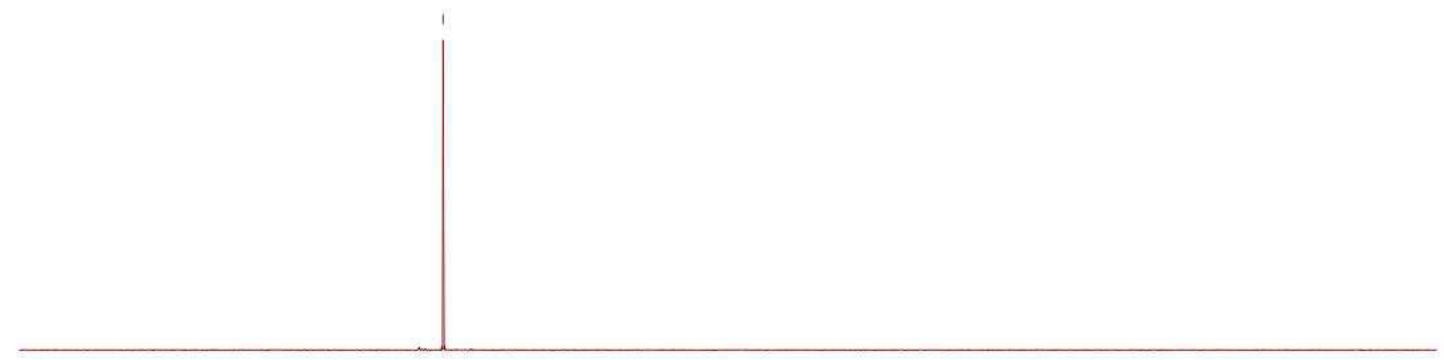

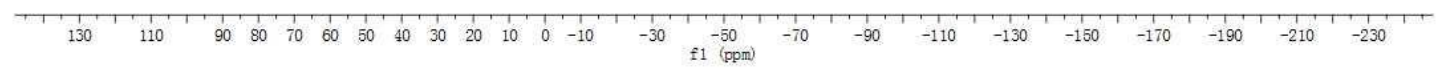




\section{${ }^{1} \mathrm{H}$ NMR of $\mathbf{3 d}$}

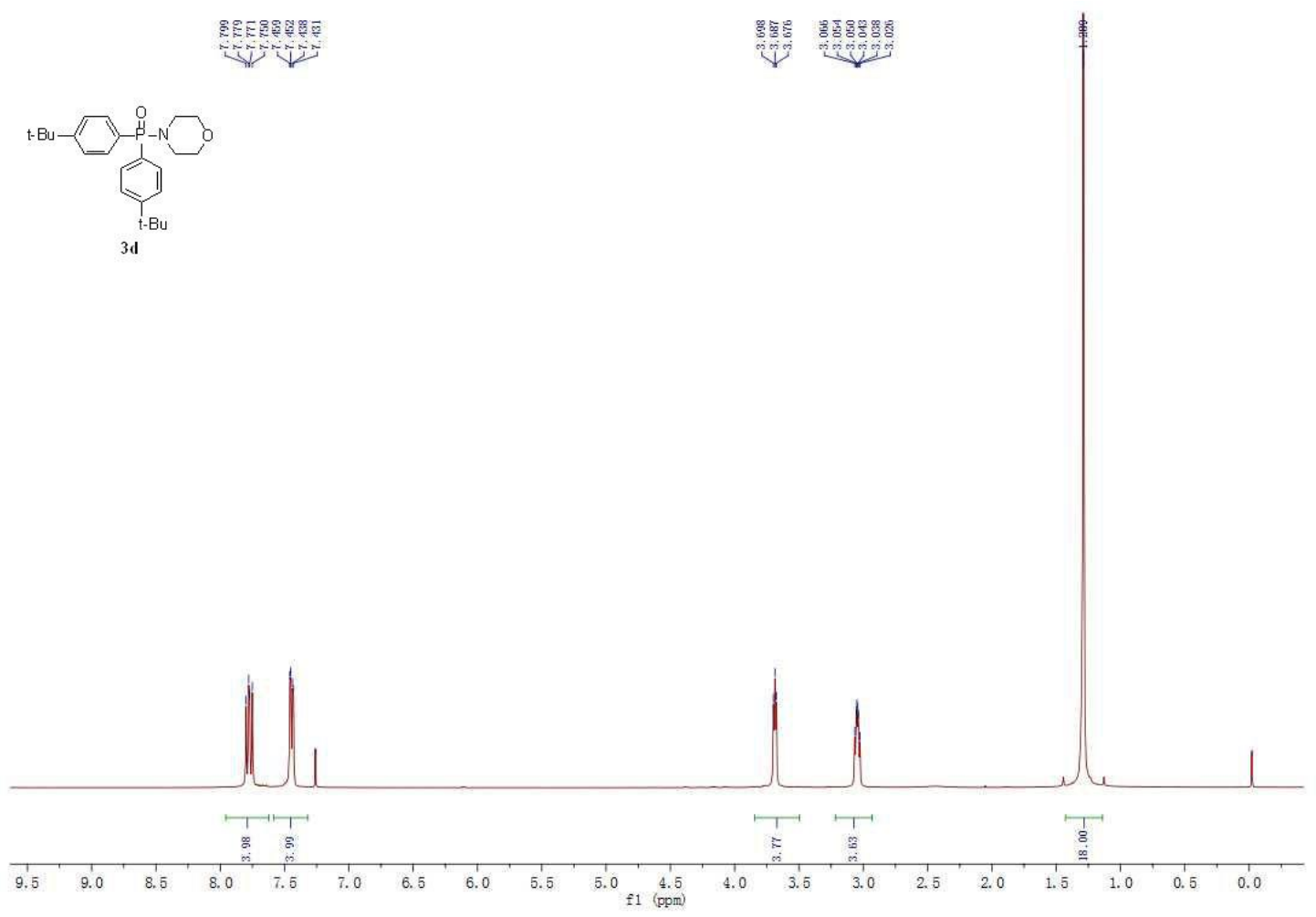

${ }^{13} \mathrm{C}$ NMR of $\mathbf{3 d}$

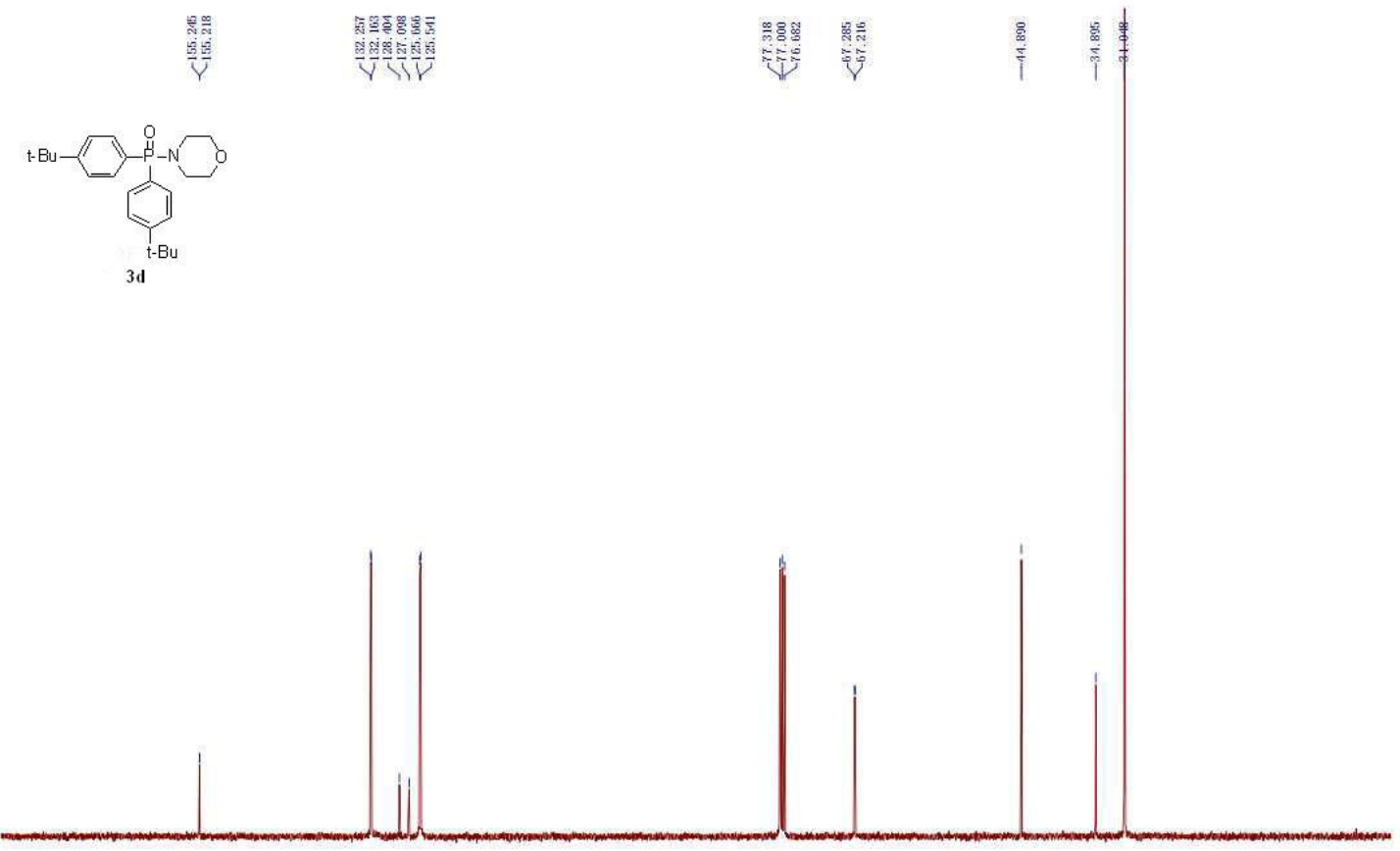

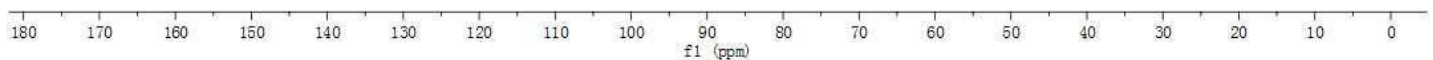


${ }^{31}$ P NMR of 3d

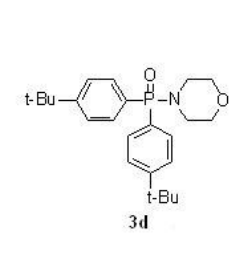

đad

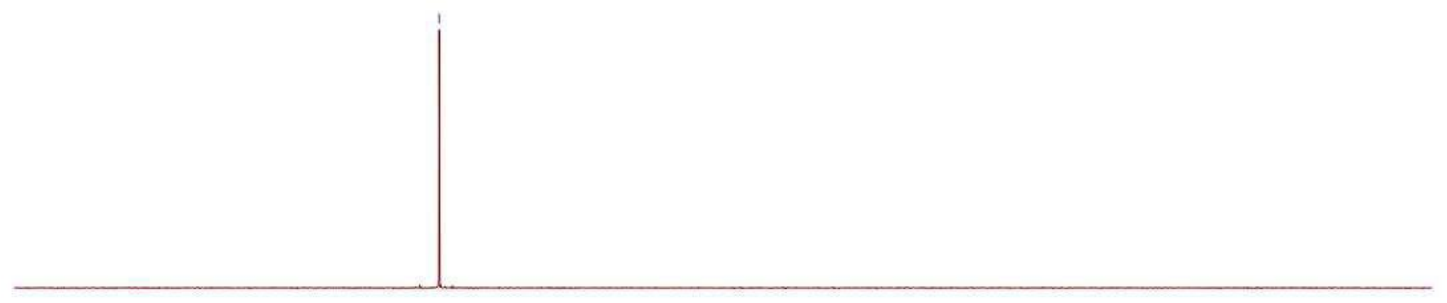

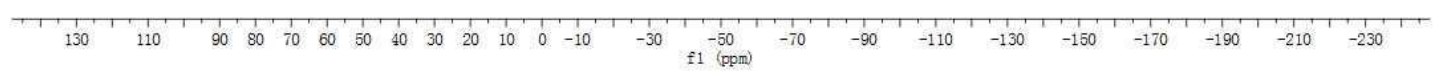

${ }^{1} \mathrm{H}$ NMR of $\mathbf{3 e}$

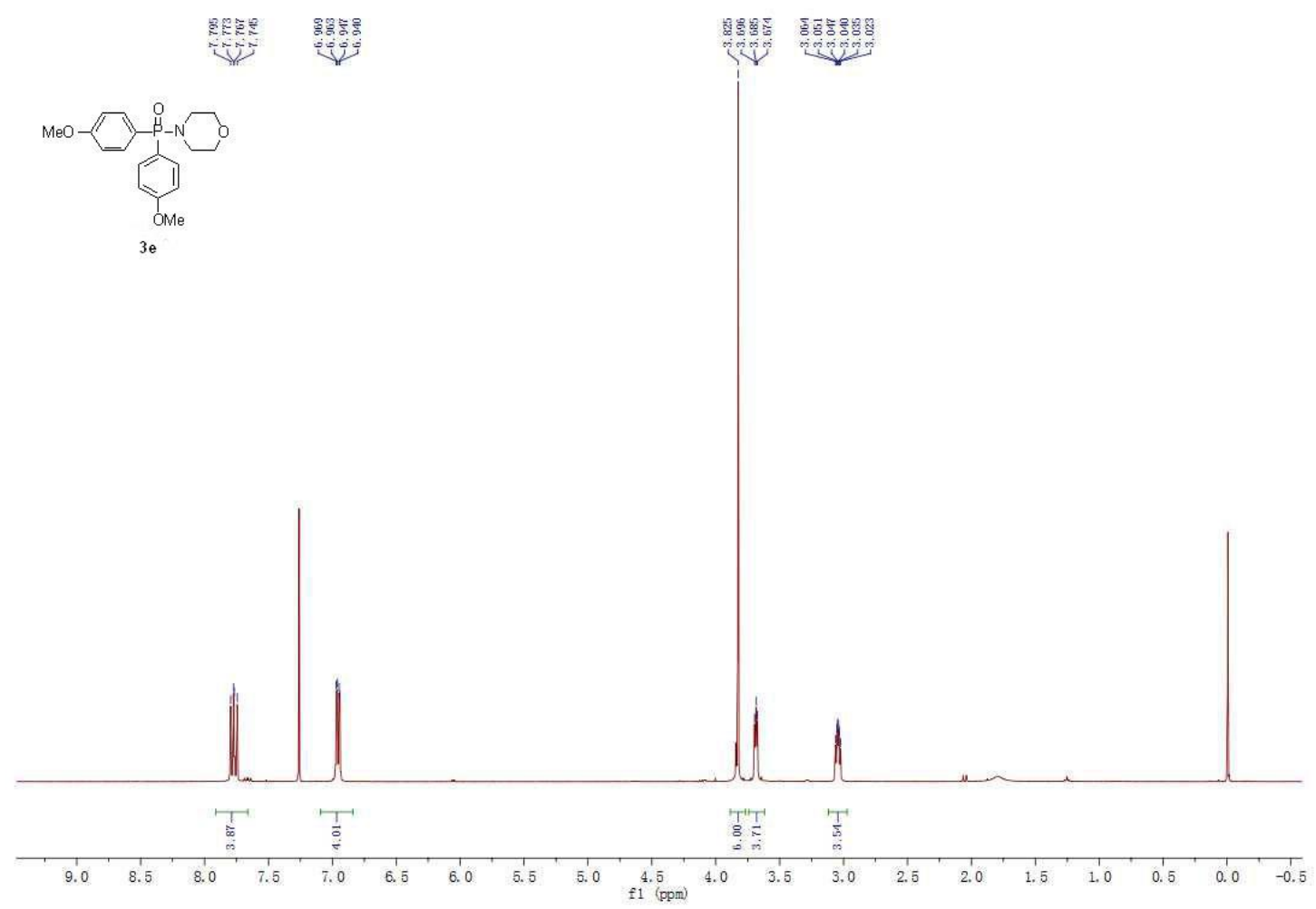


${ }^{13} \mathrm{C}$ NMR of $\mathbf{3 e}$

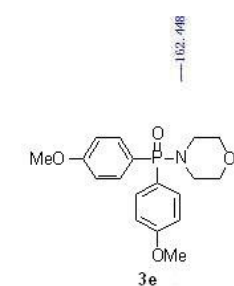

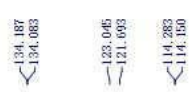

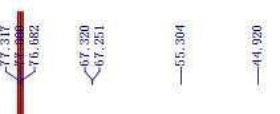

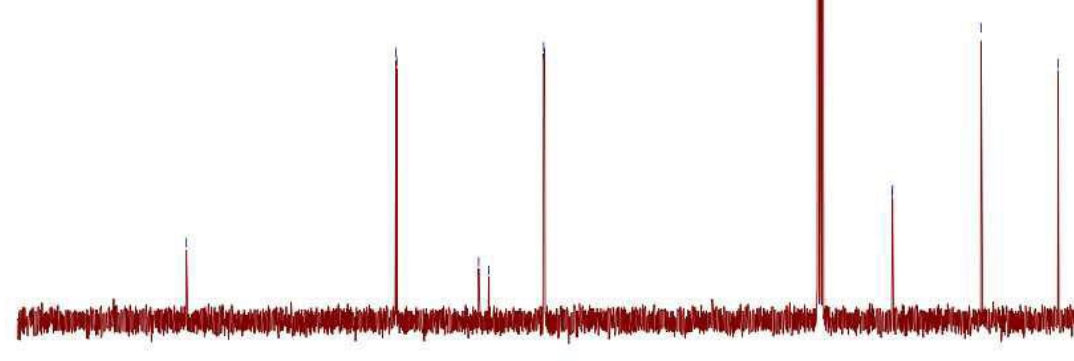

$$
180
$$

${ }^{31} \mathrm{P}$ NMR of $\mathbf{3 e}$
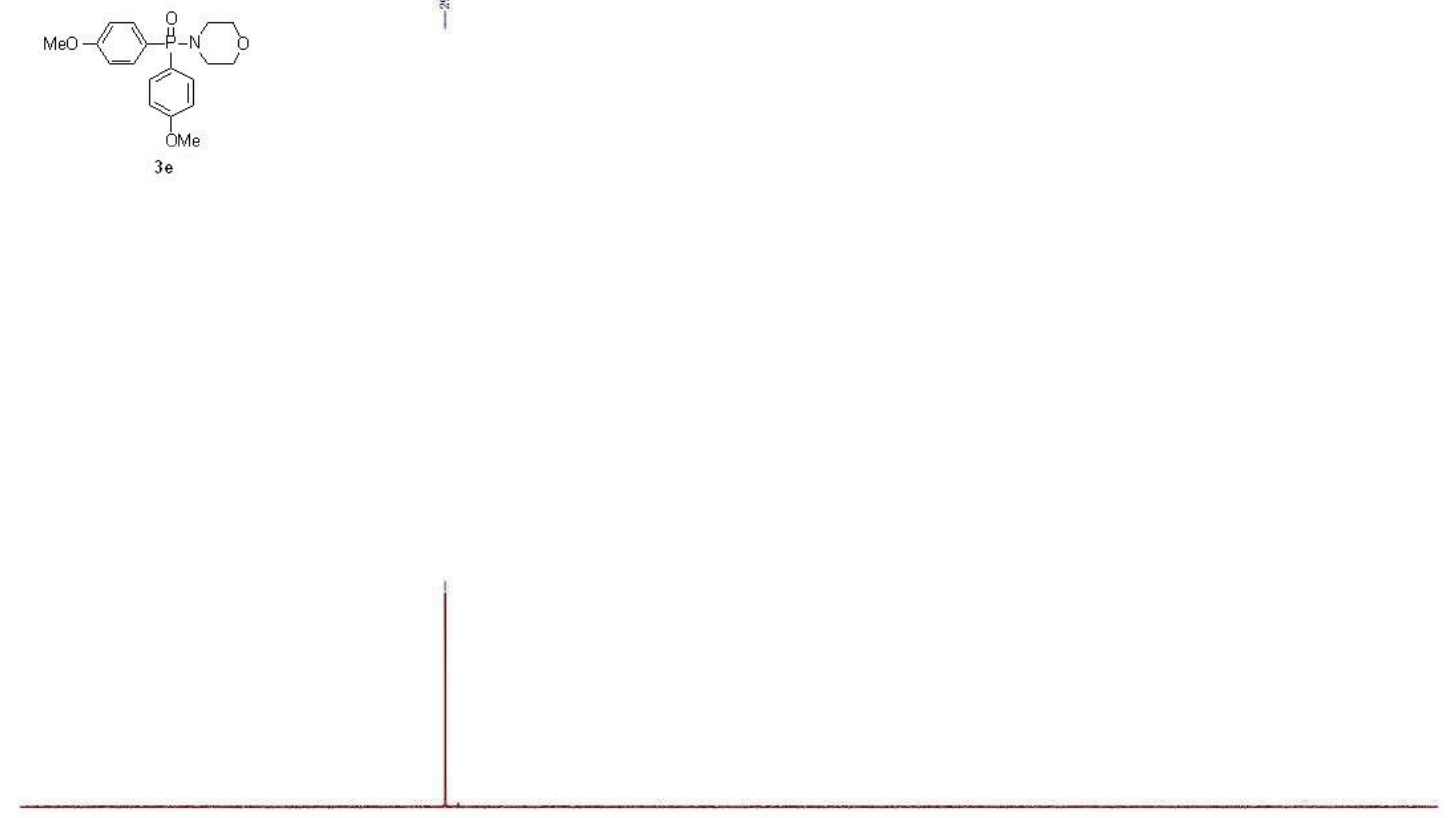

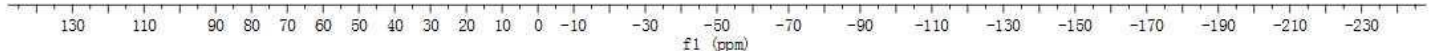




\section{${ }^{1} \mathrm{H}$ NMR of $\mathbf{3 f}$}

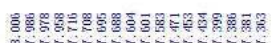

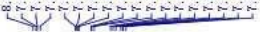

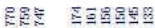

11
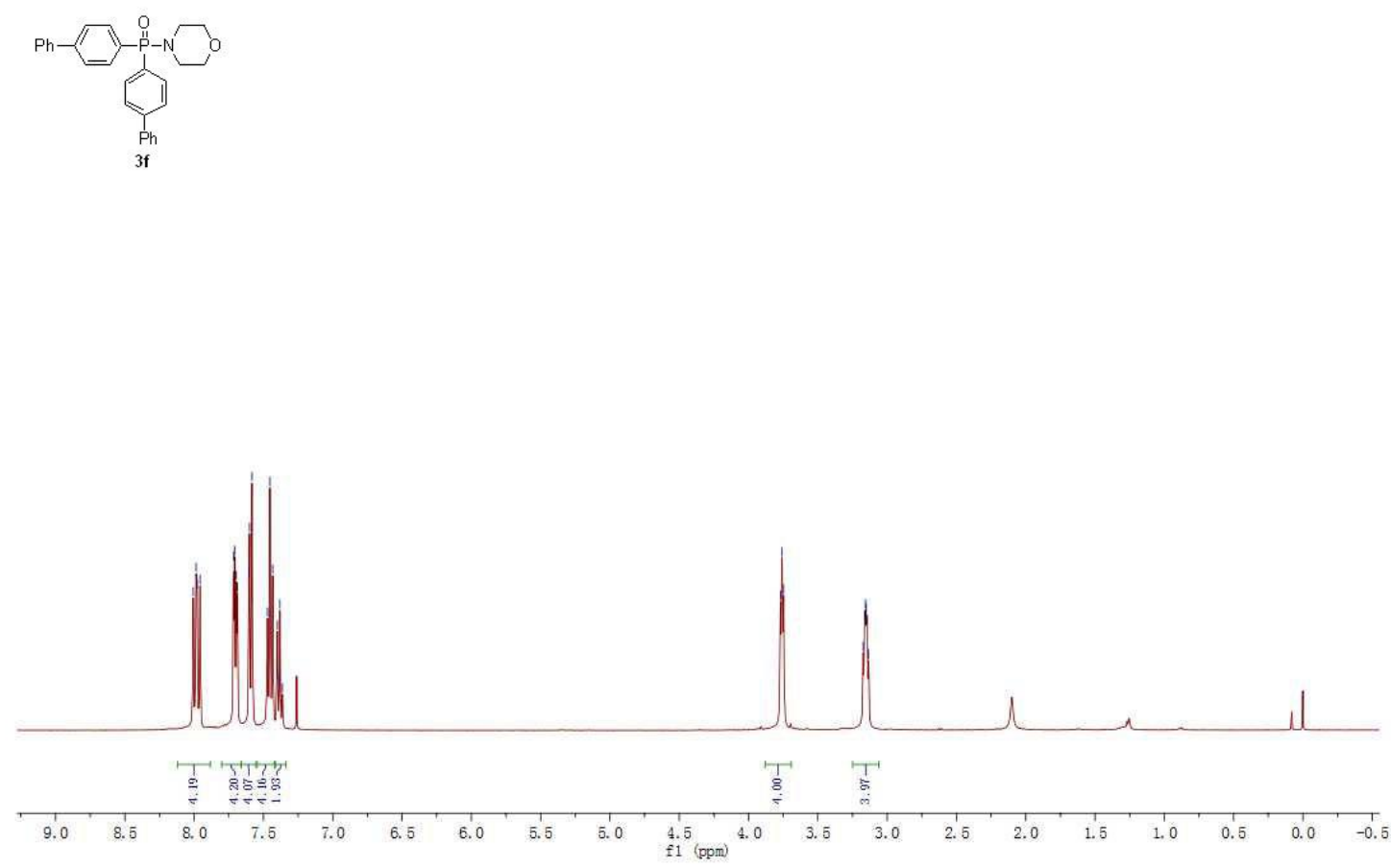

${ }^{13} \mathrm{C}$ NMR of $\mathbf{3 f}$

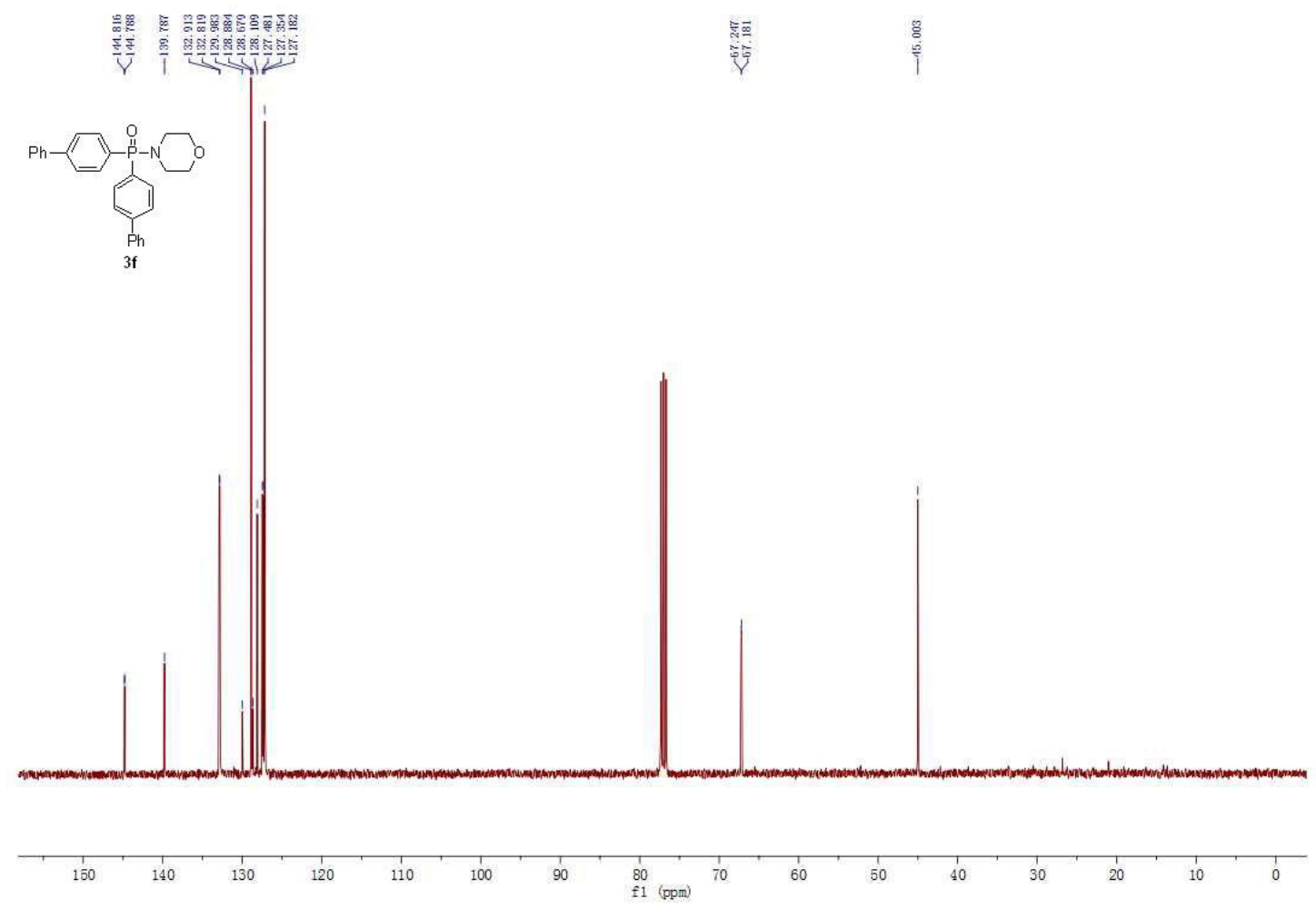


${ }^{31} \mathrm{P}$ NMR of $\mathbf{3 f}$

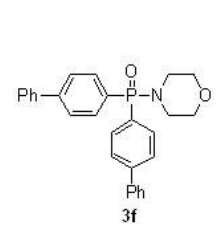

$\stackrel{\bar{N}}{i}$

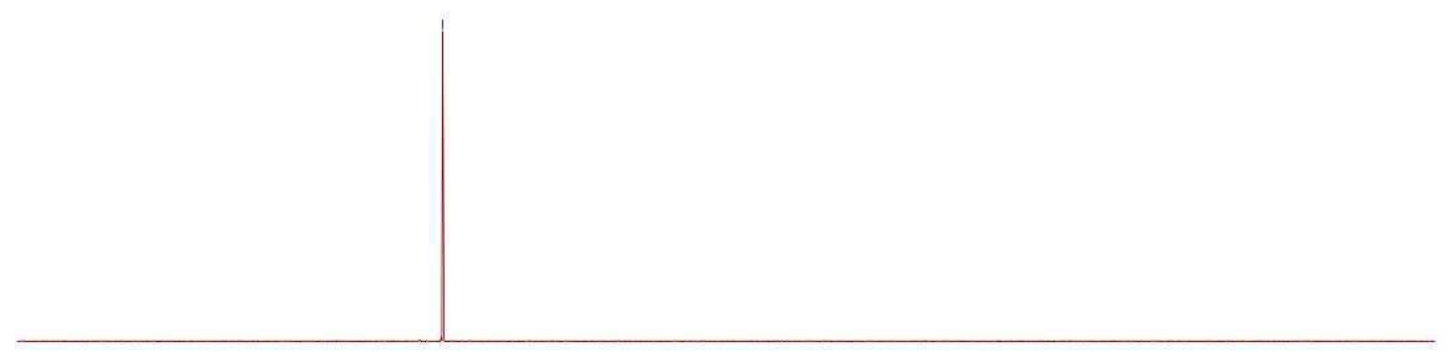

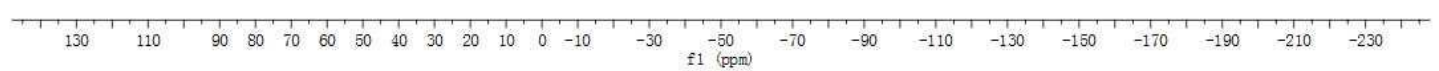

${ }^{1} \mathrm{H}$ NMR of $\mathbf{3 g}$
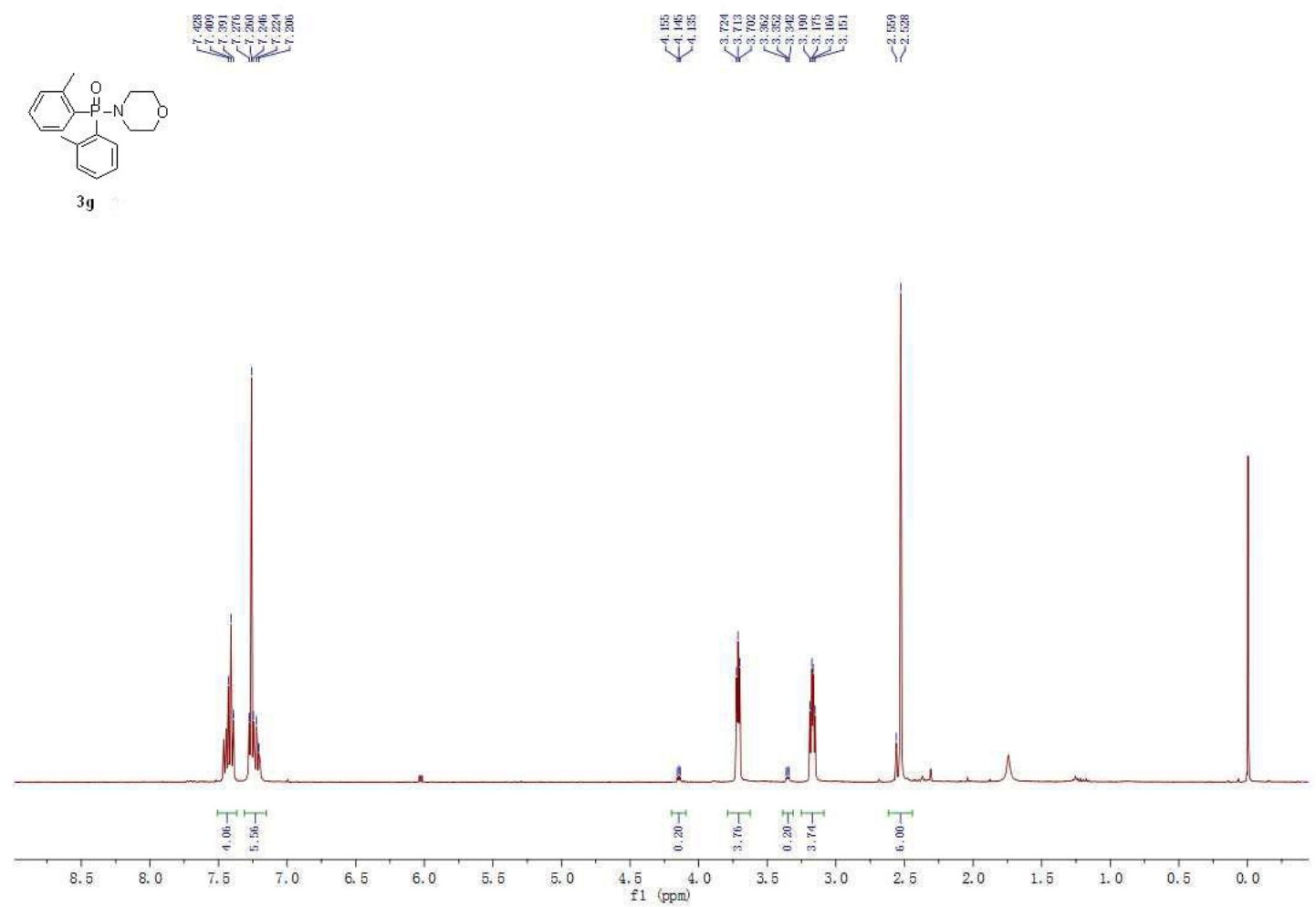
${ }^{13} \mathrm{C}$ NMR of $\mathbf{3 g}$
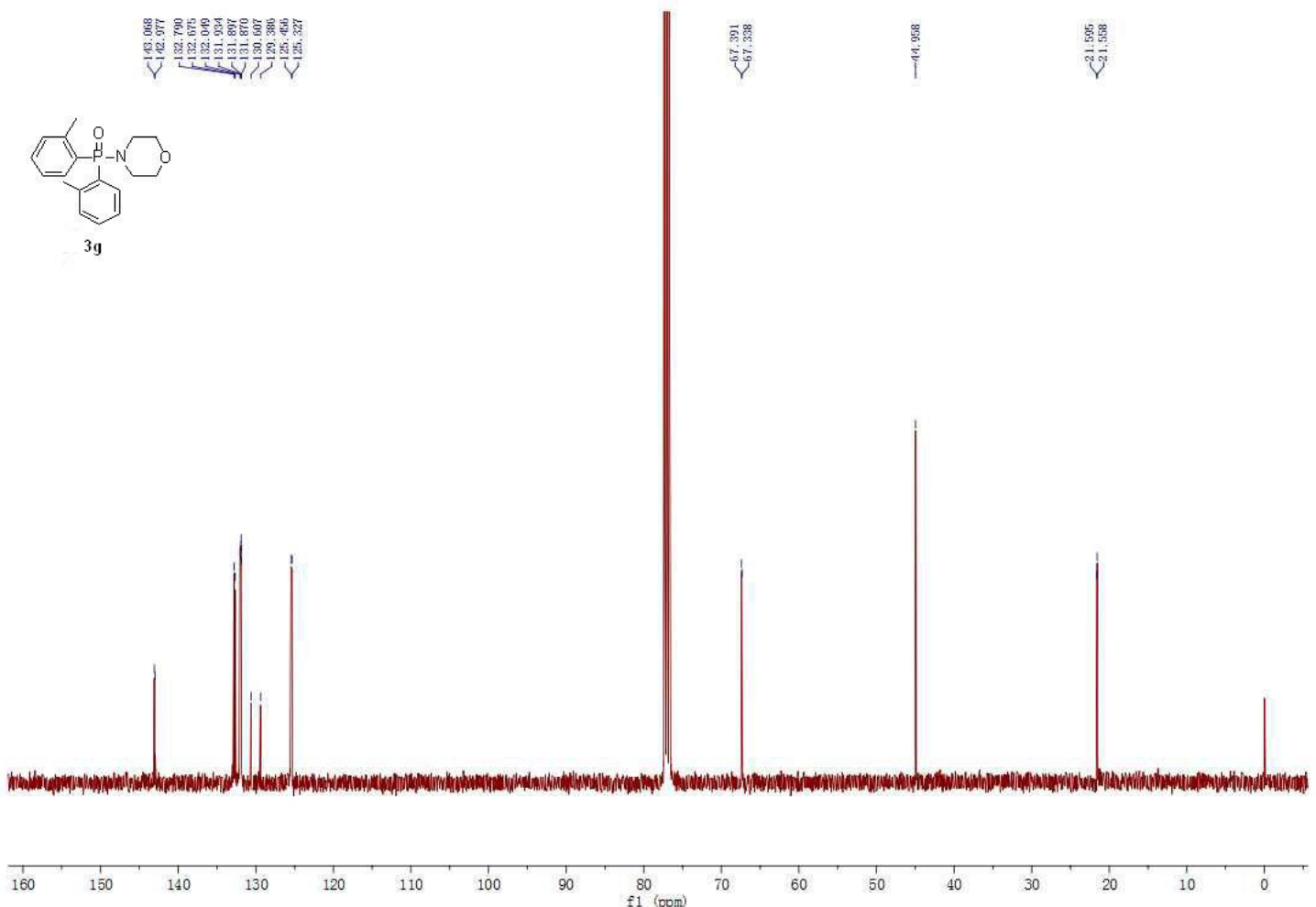

${ }^{31} \mathrm{P}$ NMR of $\mathbf{3 g}$

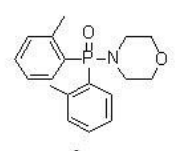

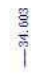

3g

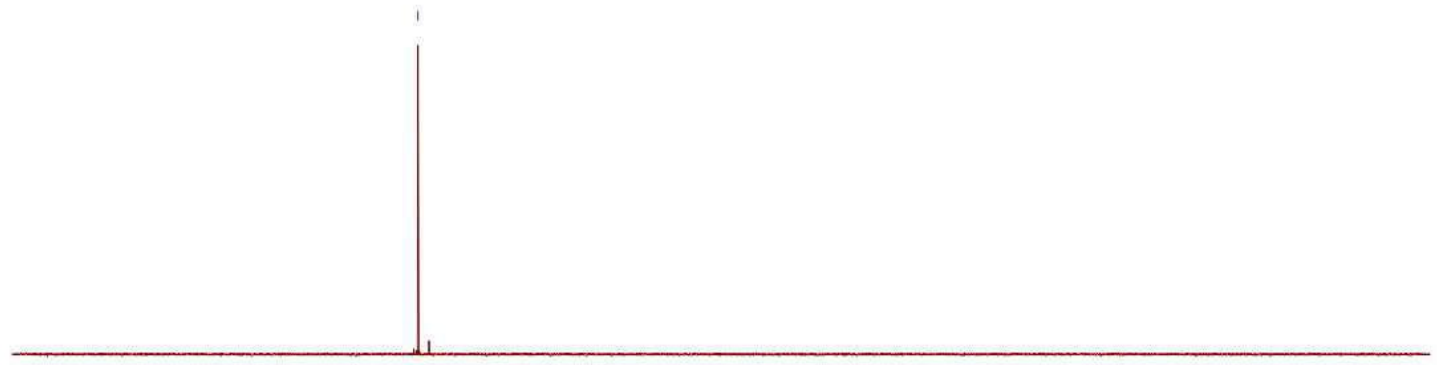

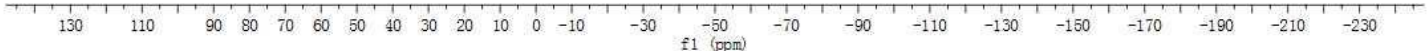


${ }^{1} \mathrm{H}$ NMR of $\mathbf{3 h}$

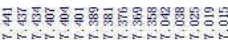

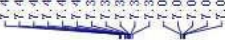


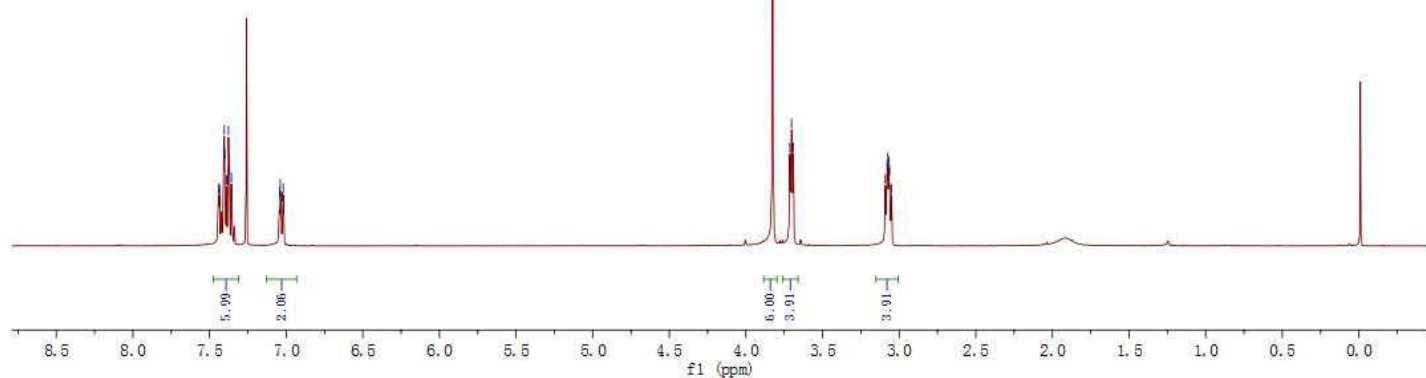

${ }^{13} \mathrm{C}$ NMR of $\mathbf{3 h}$
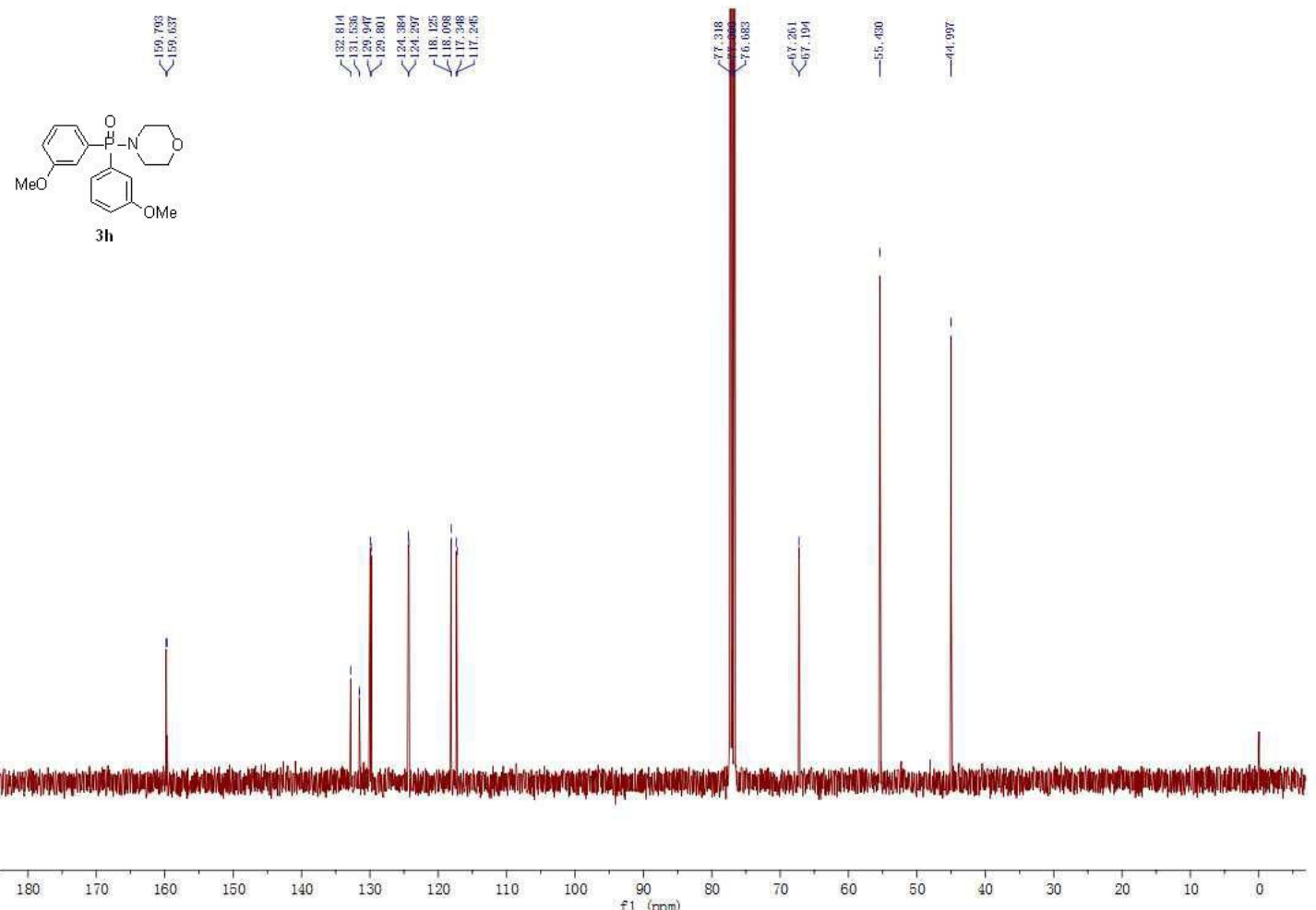
${ }^{31}$ P NMR of $\mathbf{3 h}$

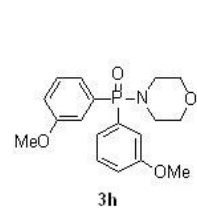

$3 \mathrm{~h}$

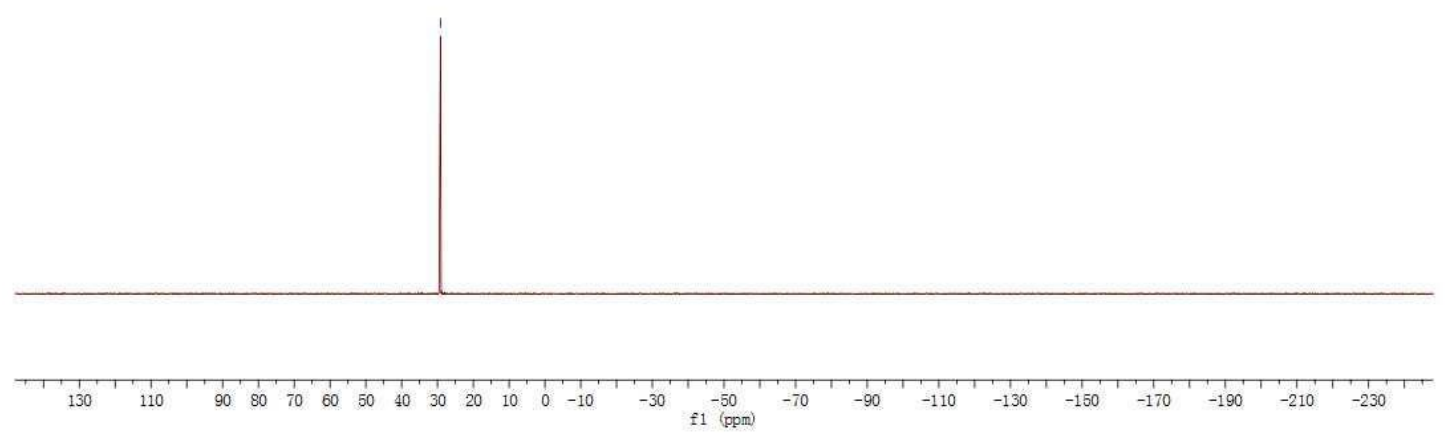

${ }^{1} \mathrm{H}$ NMR of $\mathbf{3 i}$

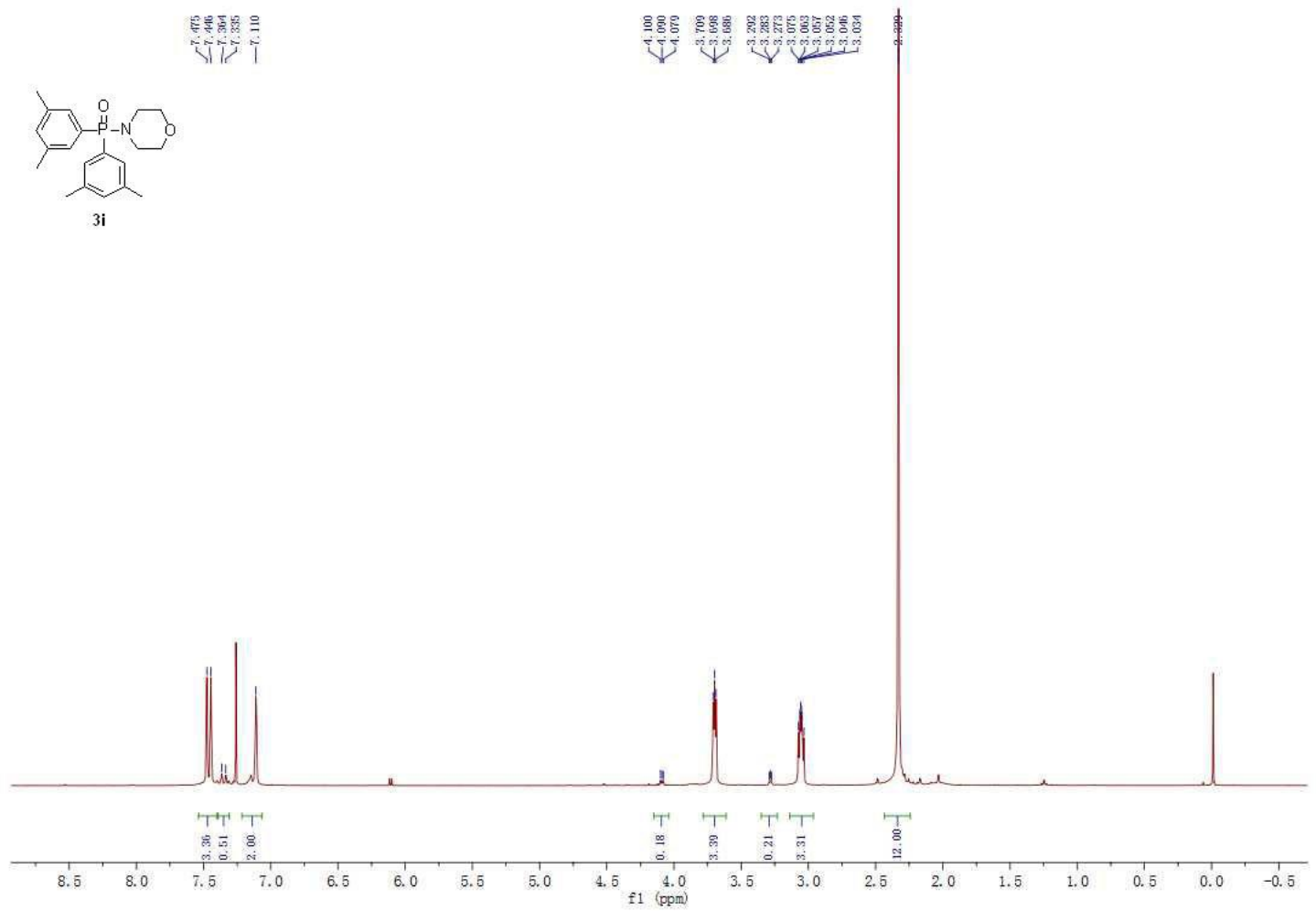


${ }^{13} \mathrm{C}$ NMR of $3 \mathbf{i}$
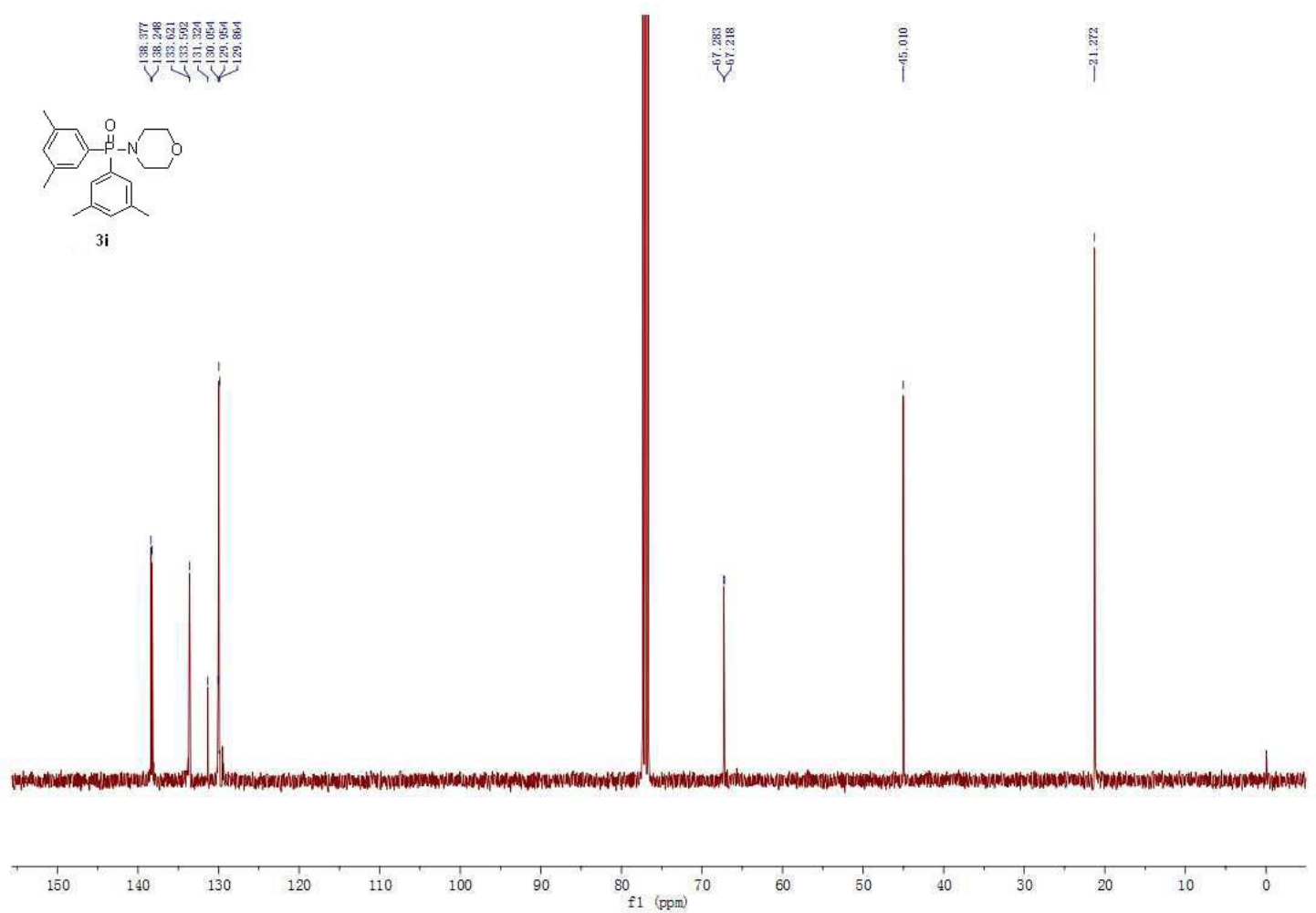

${ }^{31}$ P NMR of $\mathbf{3 i}$

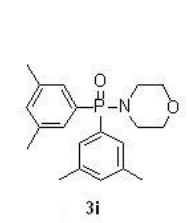

$\stackrel{2}{i}$

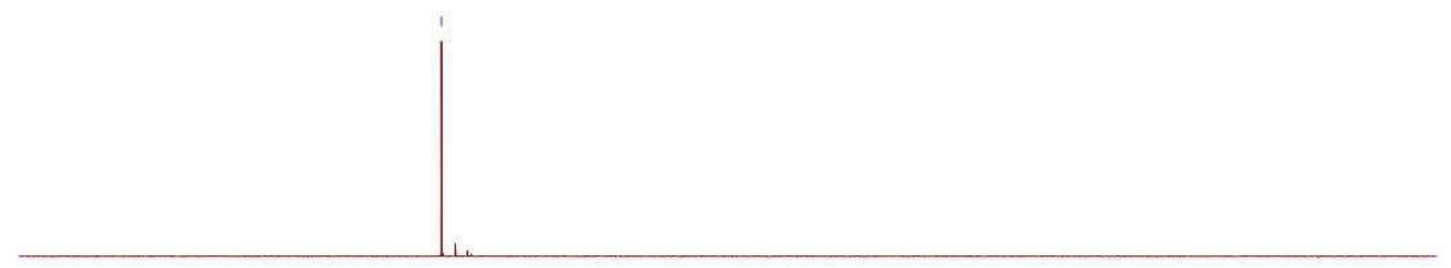

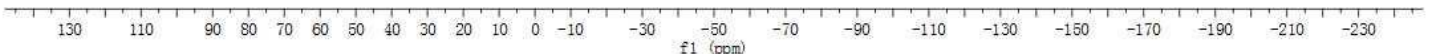


${ }^{1} \mathrm{H}$ NMR of $\mathbf{3 j}$

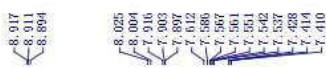
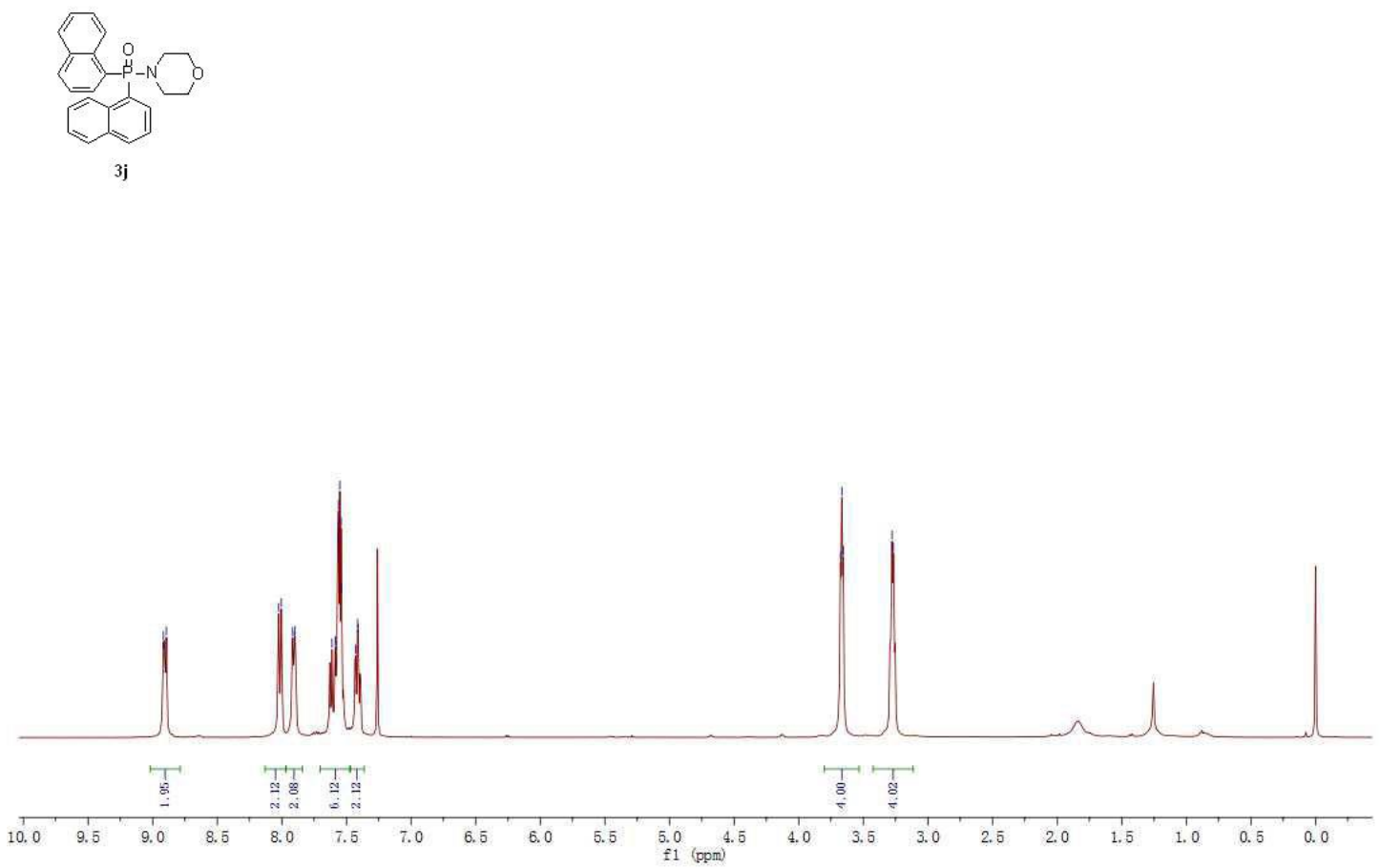

${ }^{13} \mathrm{C}$ NMR of $\mathbf{3 j}$

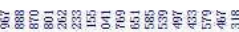

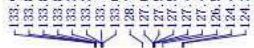

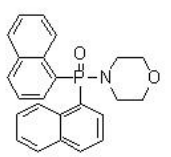

$3 \mathbf{j}$
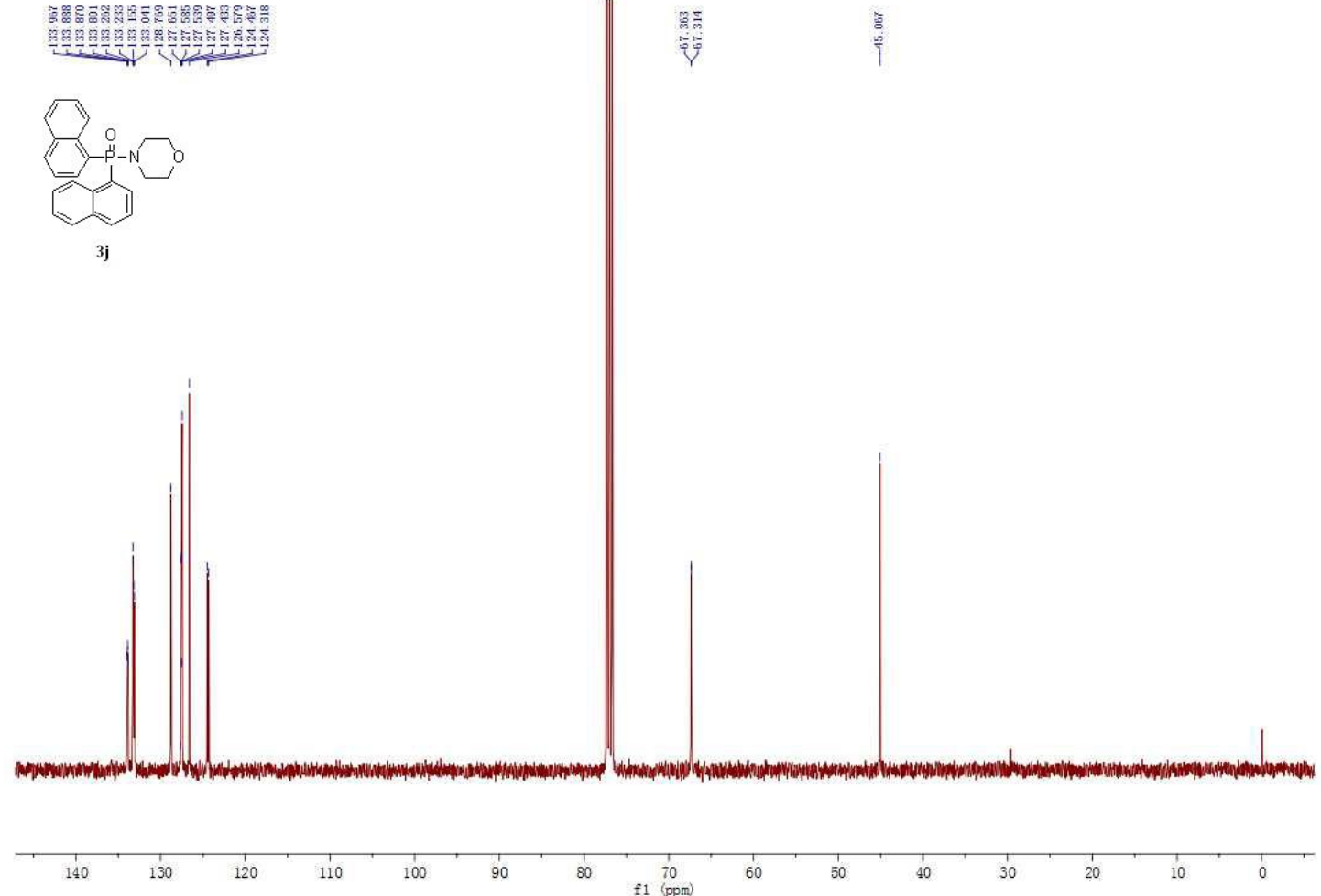
${ }^{31}$ P NMR of $\mathbf{3 j}$
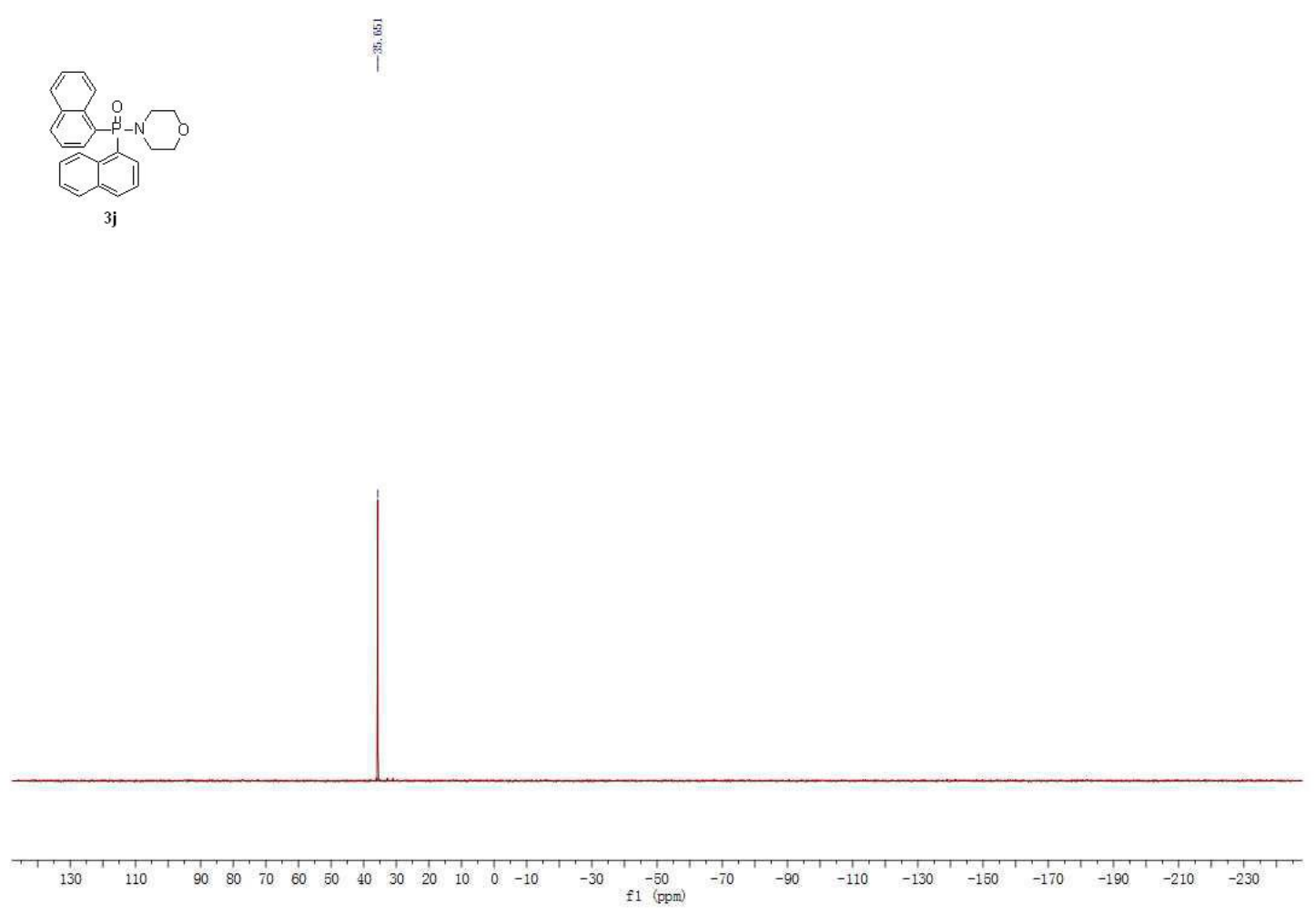

${ }^{1} \mathrm{H}$ NMR of $\mathbf{3 k}$

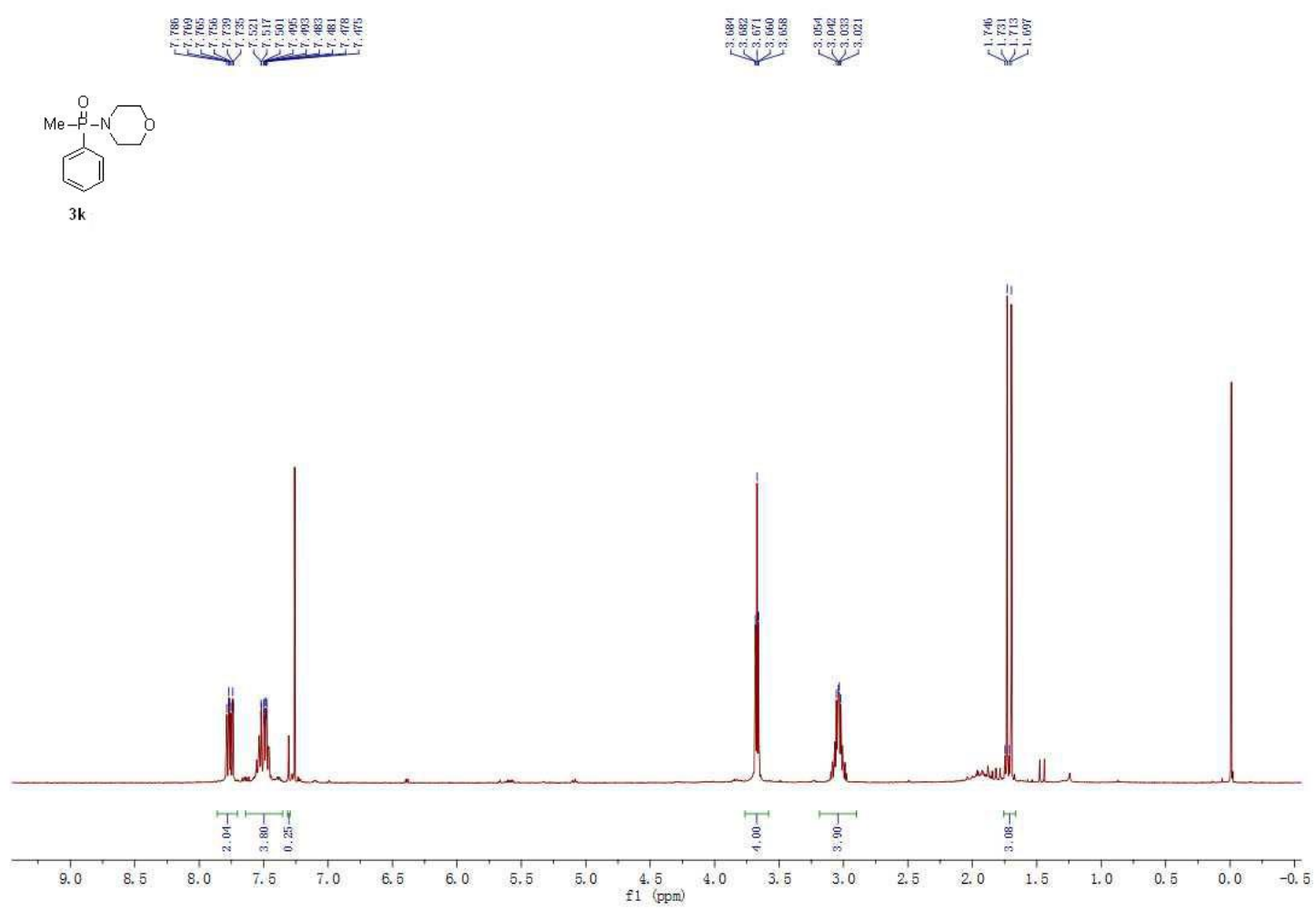


${ }^{13} \mathrm{C}$ NMR of 3k
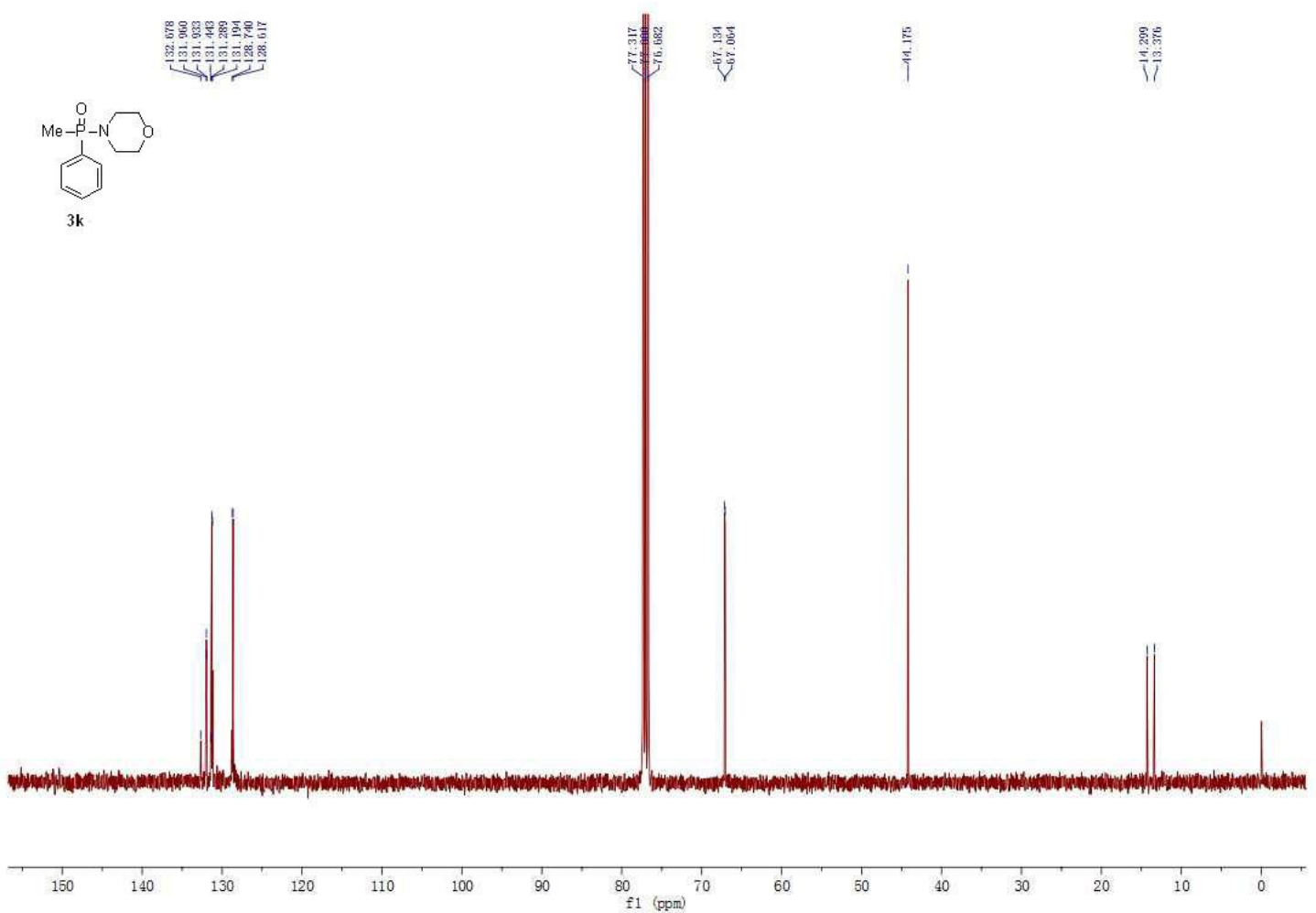

${ }^{31} \mathrm{P}$ NMR of $\mathbf{3 k}$
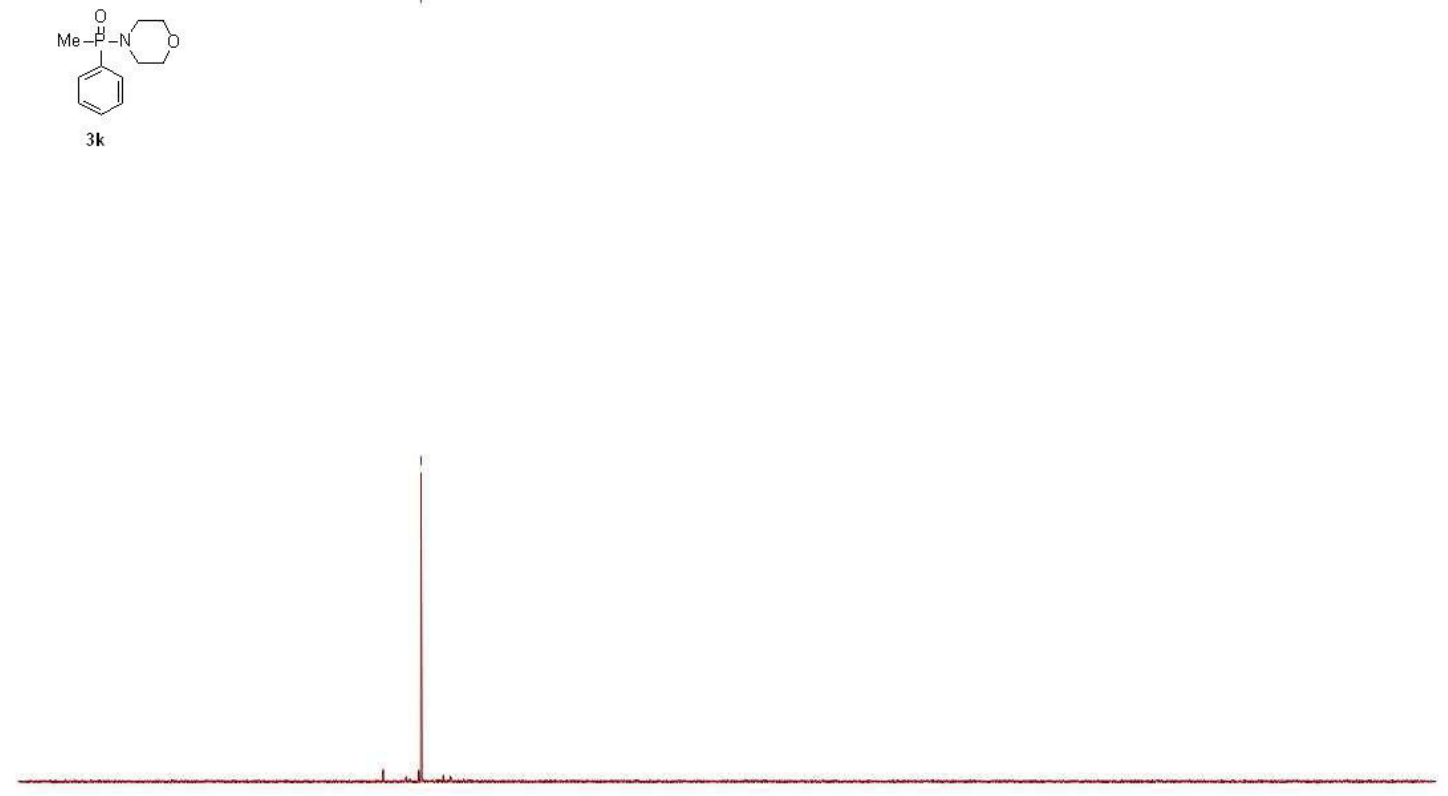

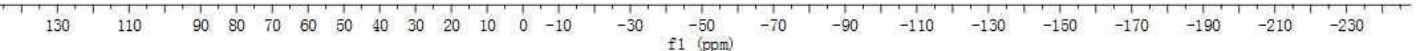


${ }^{1} \mathrm{H}$ NMR of 3 I

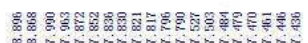

oi

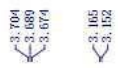

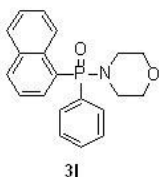

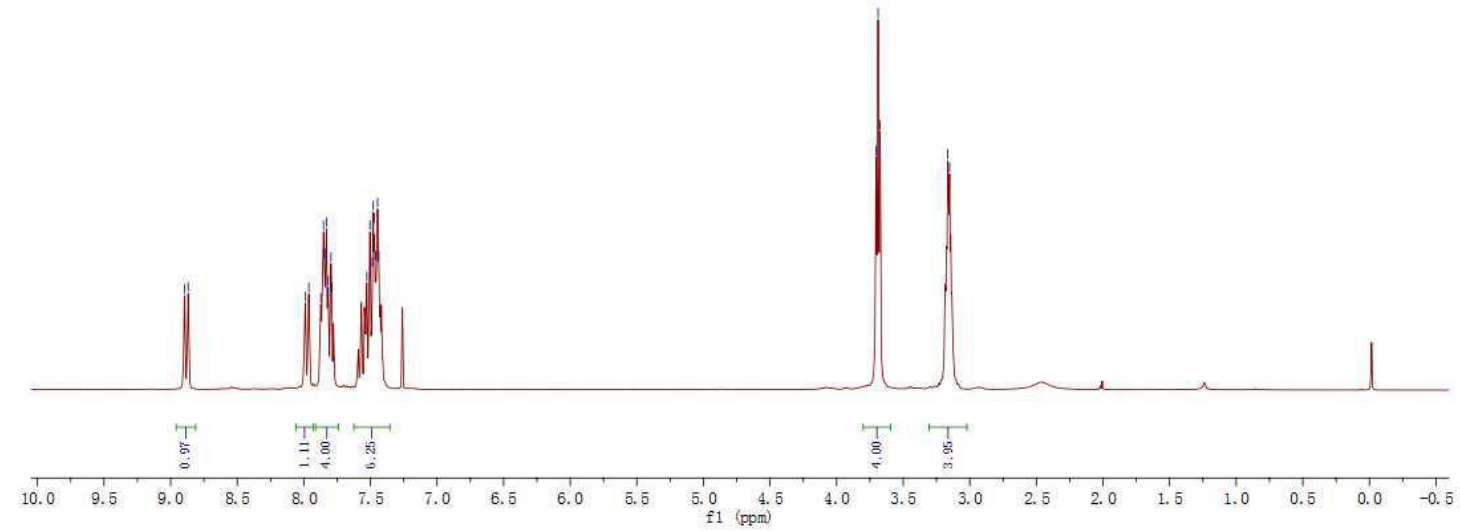

${ }^{13} \mathrm{C}$ NMR of 31

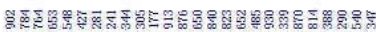

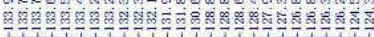

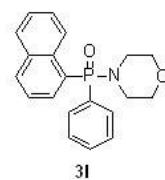

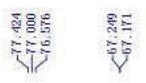

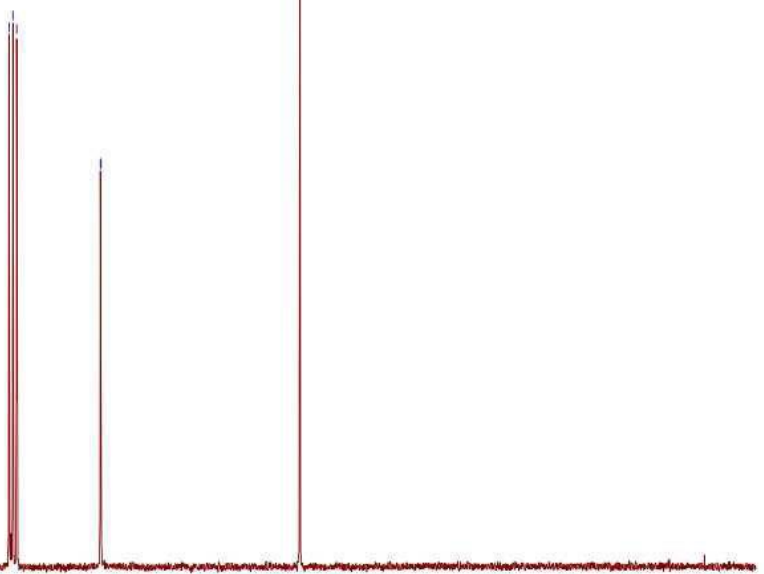

150

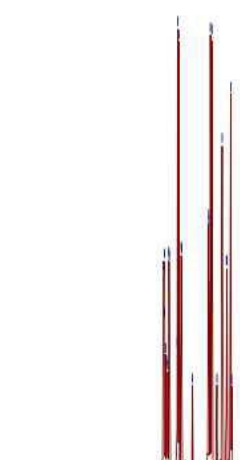

\&1 70

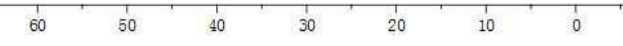


${ }^{31} \mathrm{P}$ NMR of $\mathbf{3 l}$
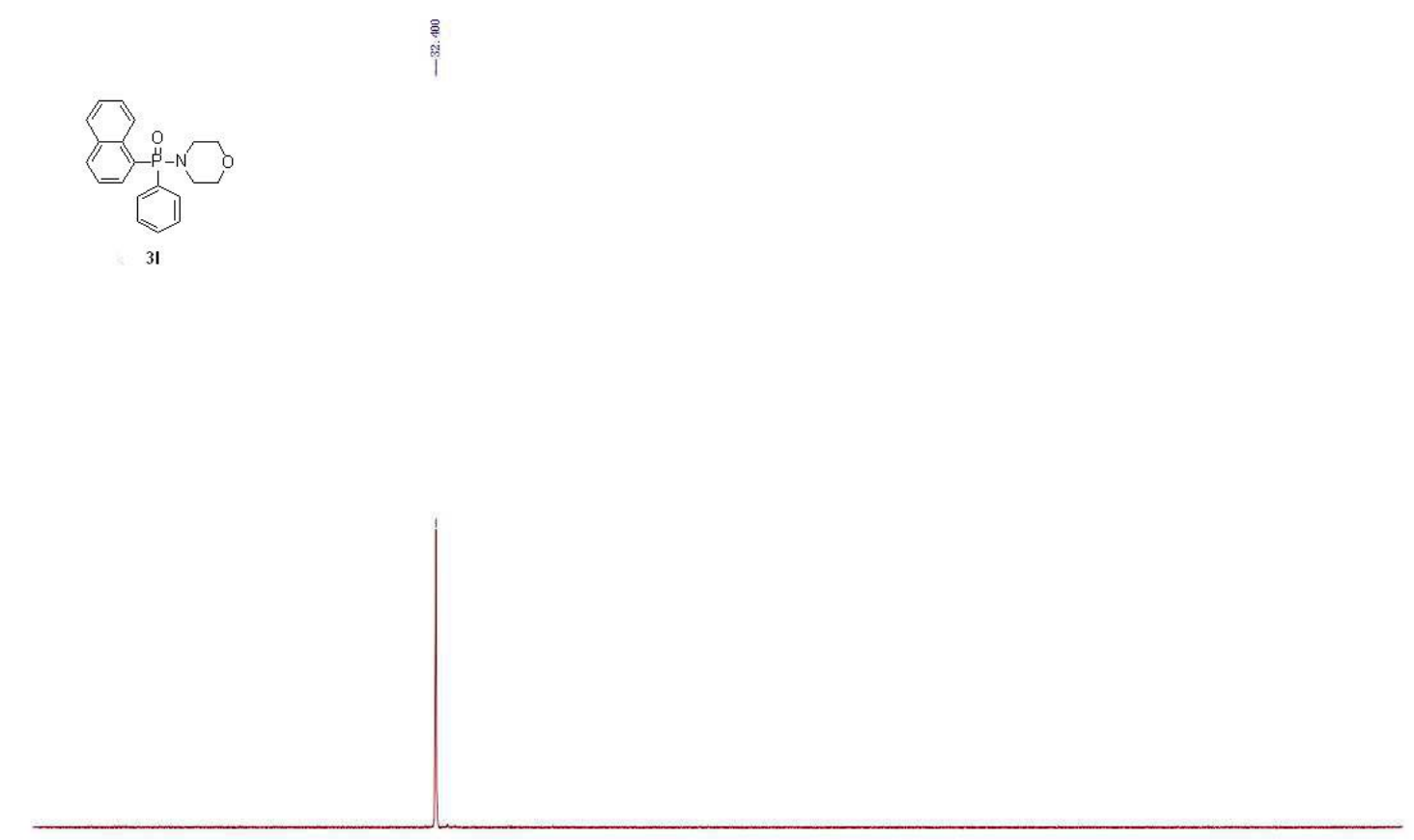

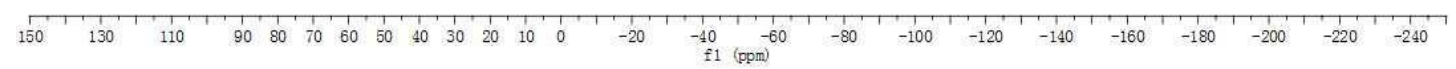

\section{${ }^{1} \mathrm{H}$ NMR of $\mathbf{3 m}$}

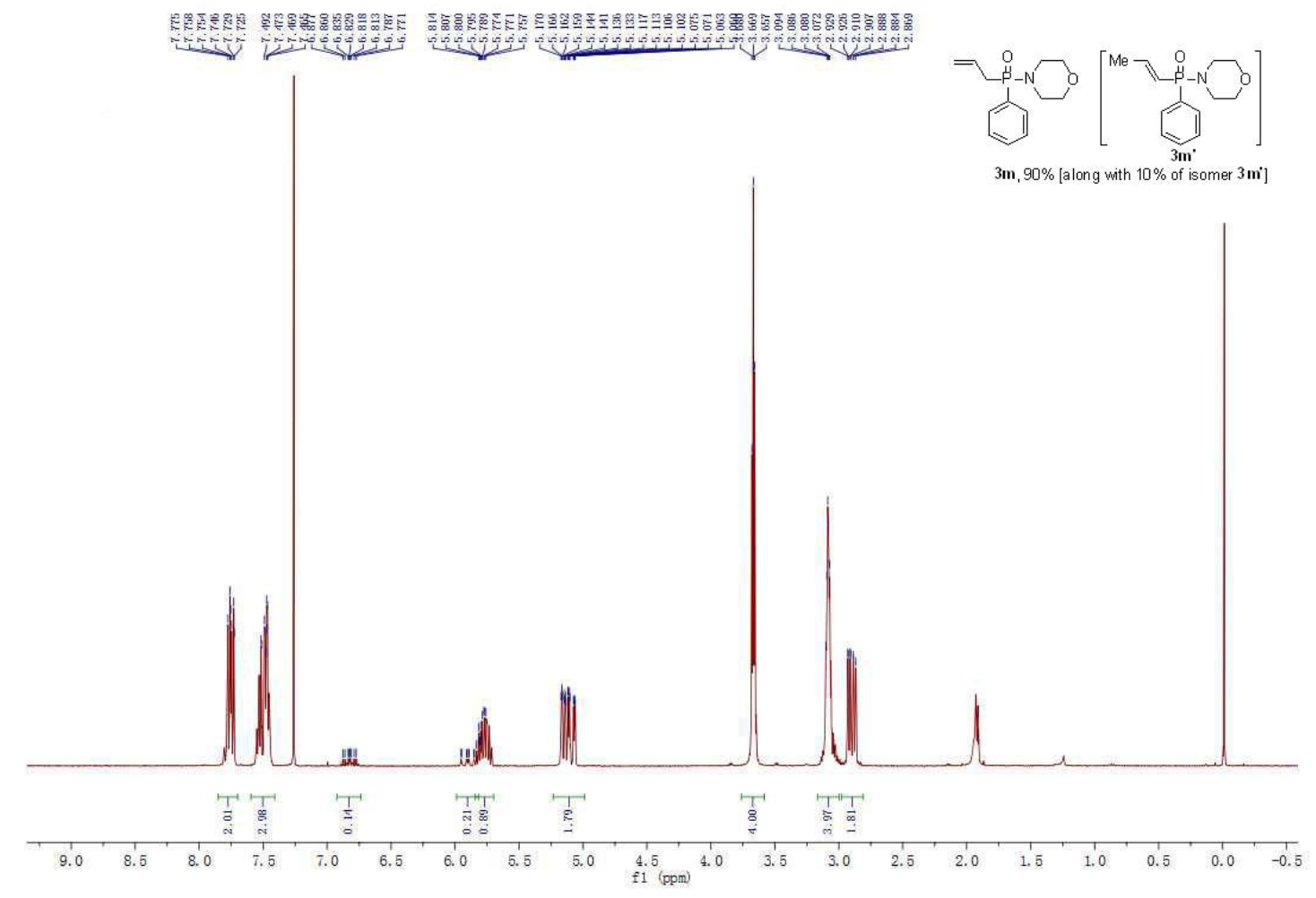


${ }^{13} \mathrm{C}$ NMR of $3 \mathbf{m}$
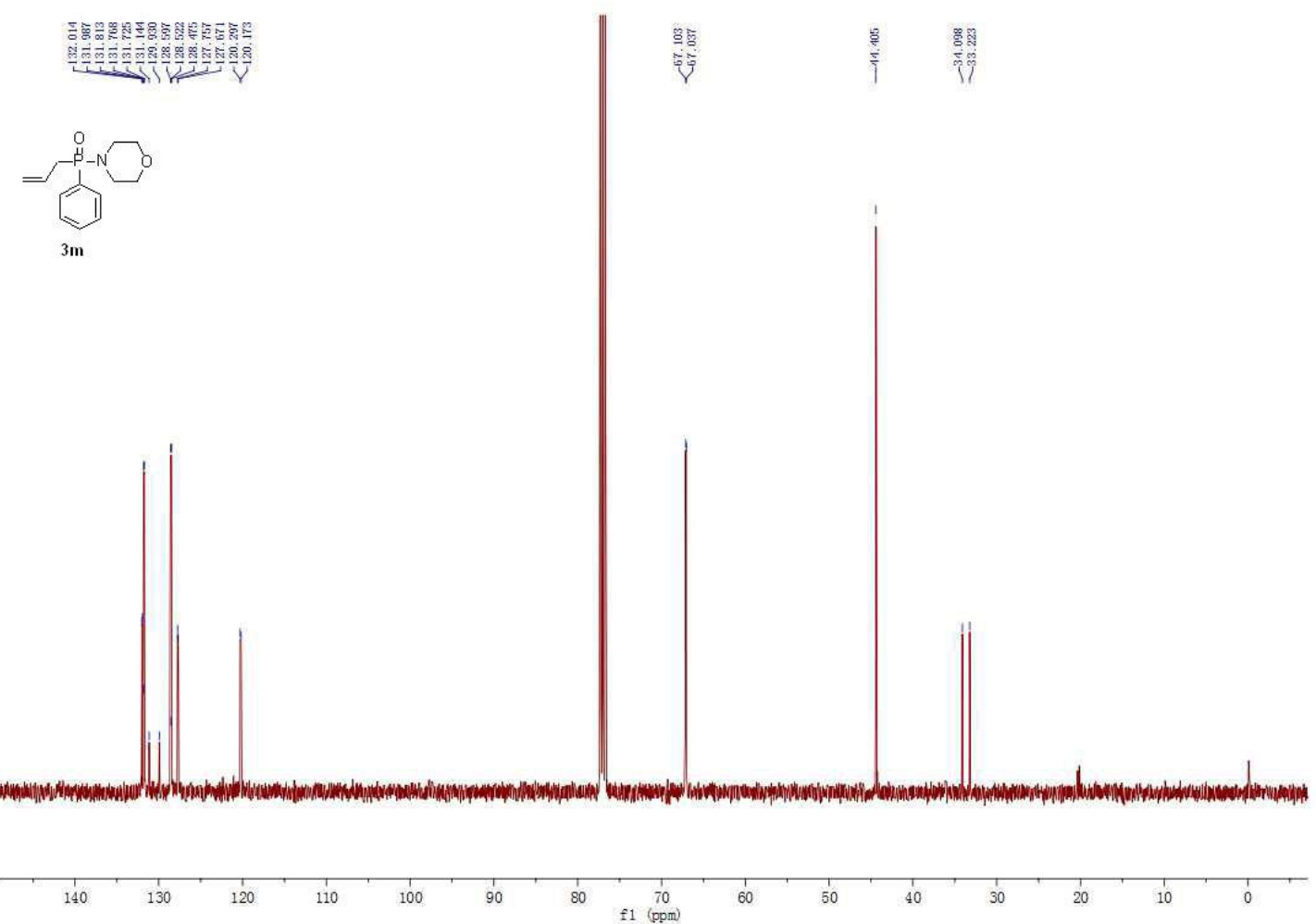

${ }^{31} \mathrm{P}$ NMR of 3m

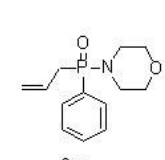

$3 \mathrm{~m}$

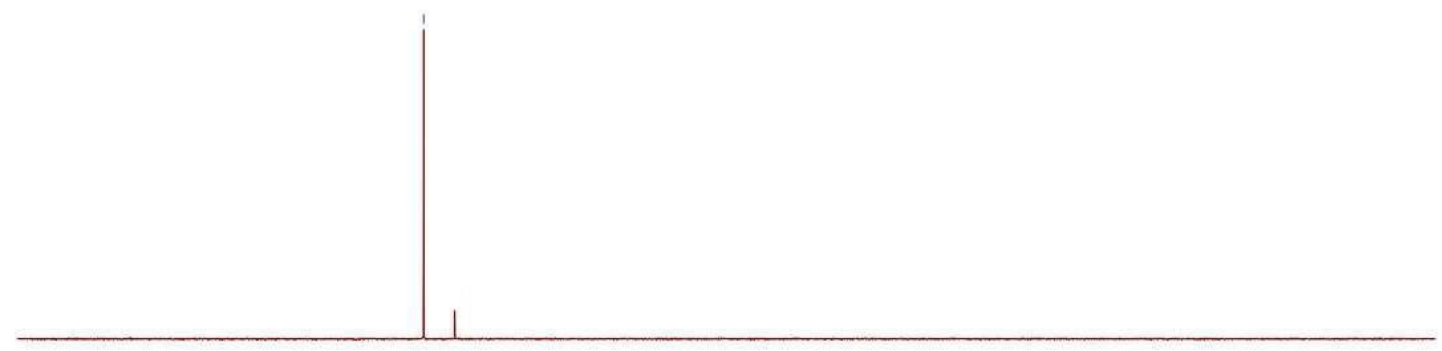

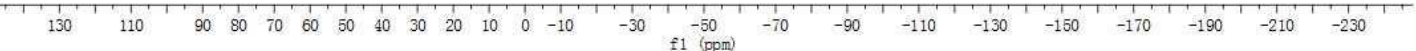




\section{${ }^{1} \mathrm{H}$ NMR of $\mathbf{3 n}$}

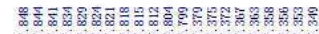

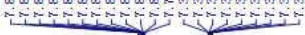

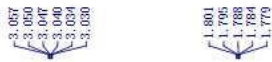

(1)

3n

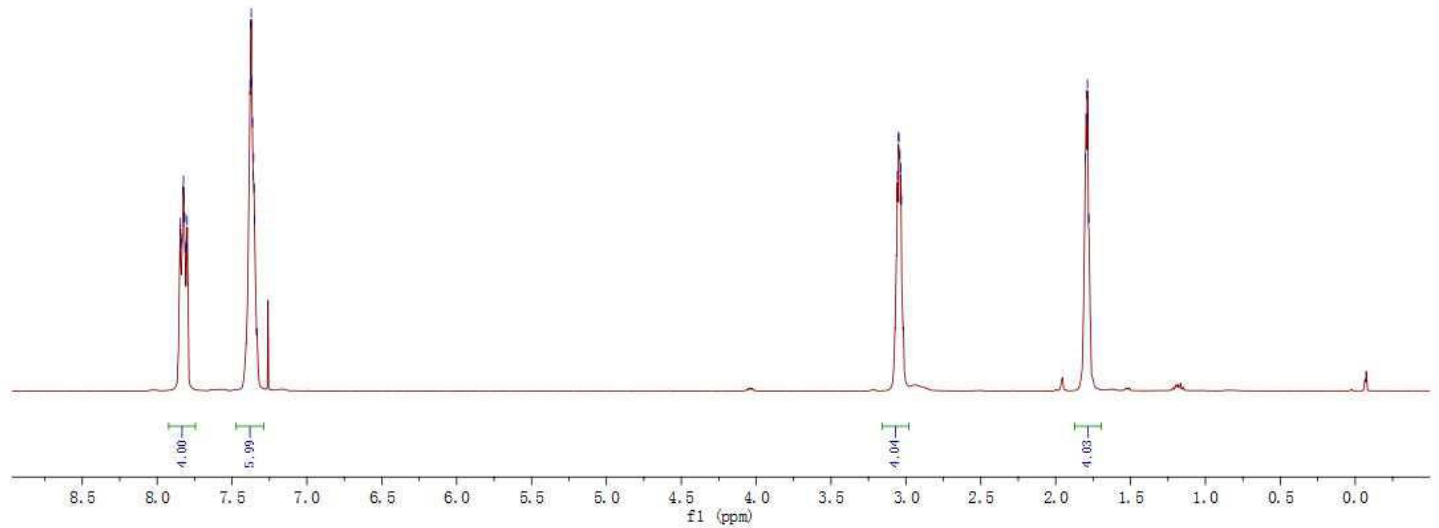

${ }^{13} \mathrm{C}$ NMR of $\mathbf{3 n}$

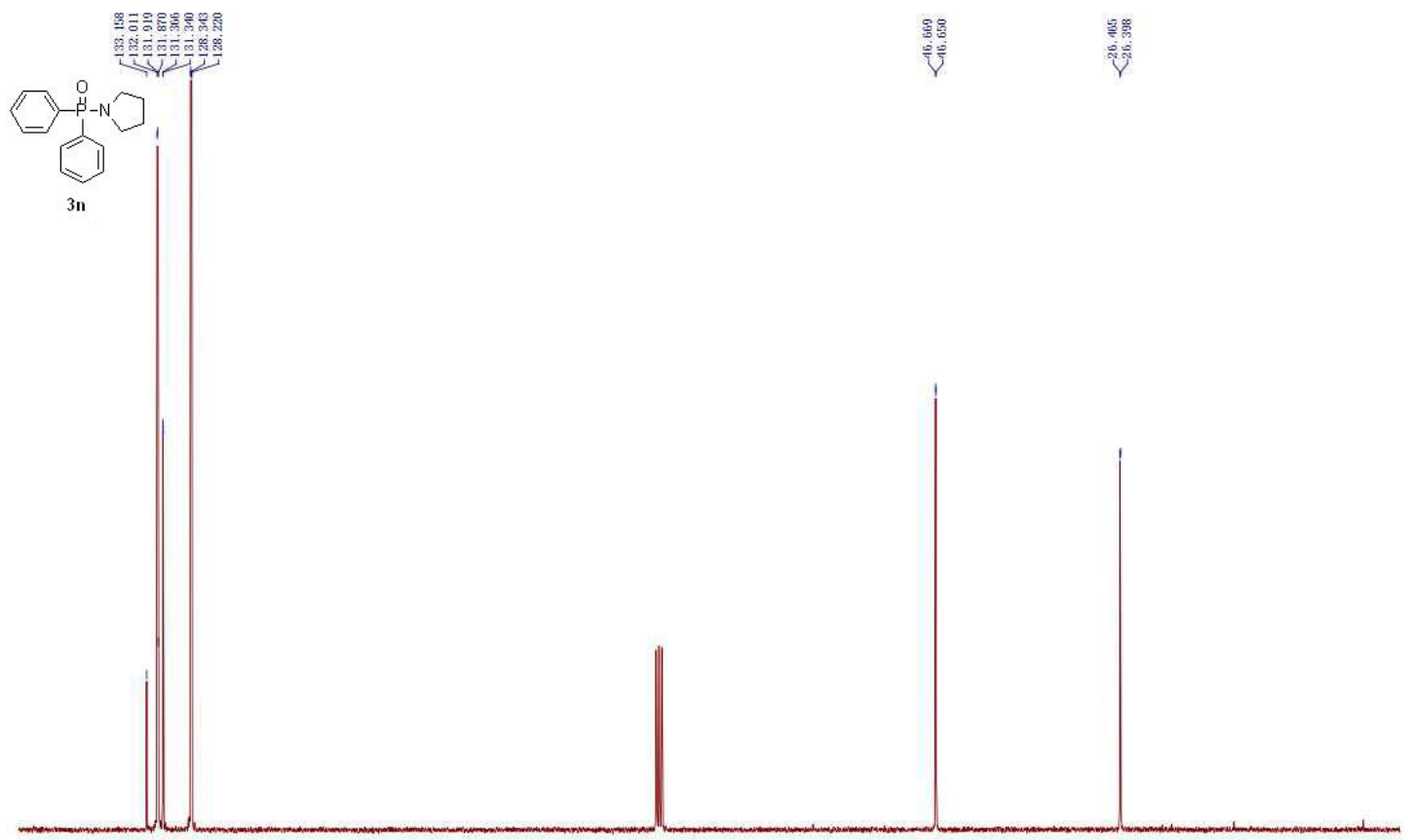

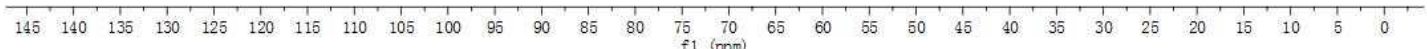


${ }^{31}$ P NMR of 3n

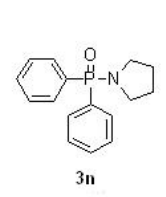

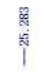

$3 n$

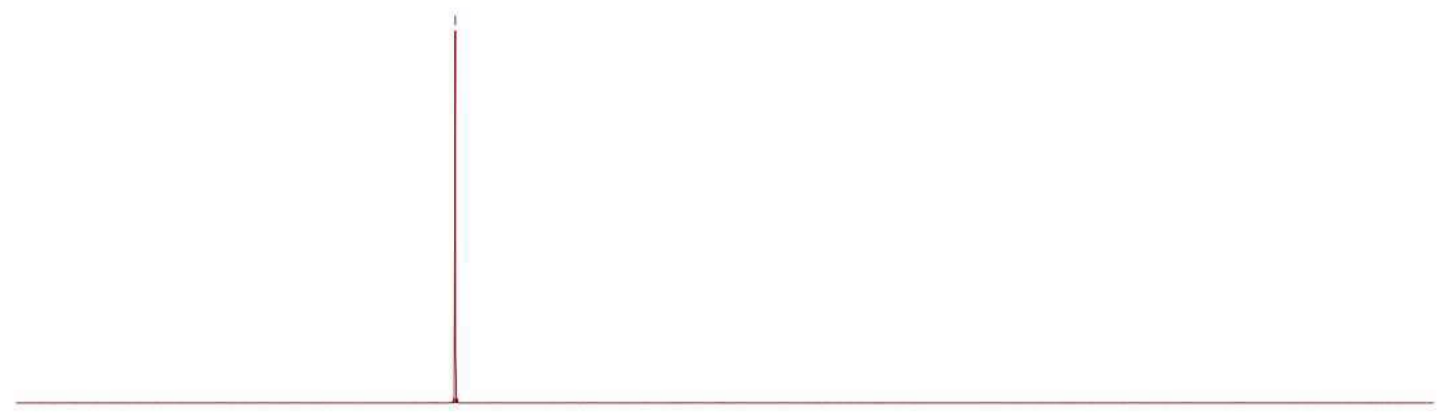

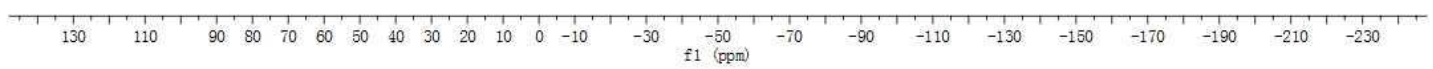

${ }^{1} \mathrm{H}$ NMR of $\mathbf{3 o}$
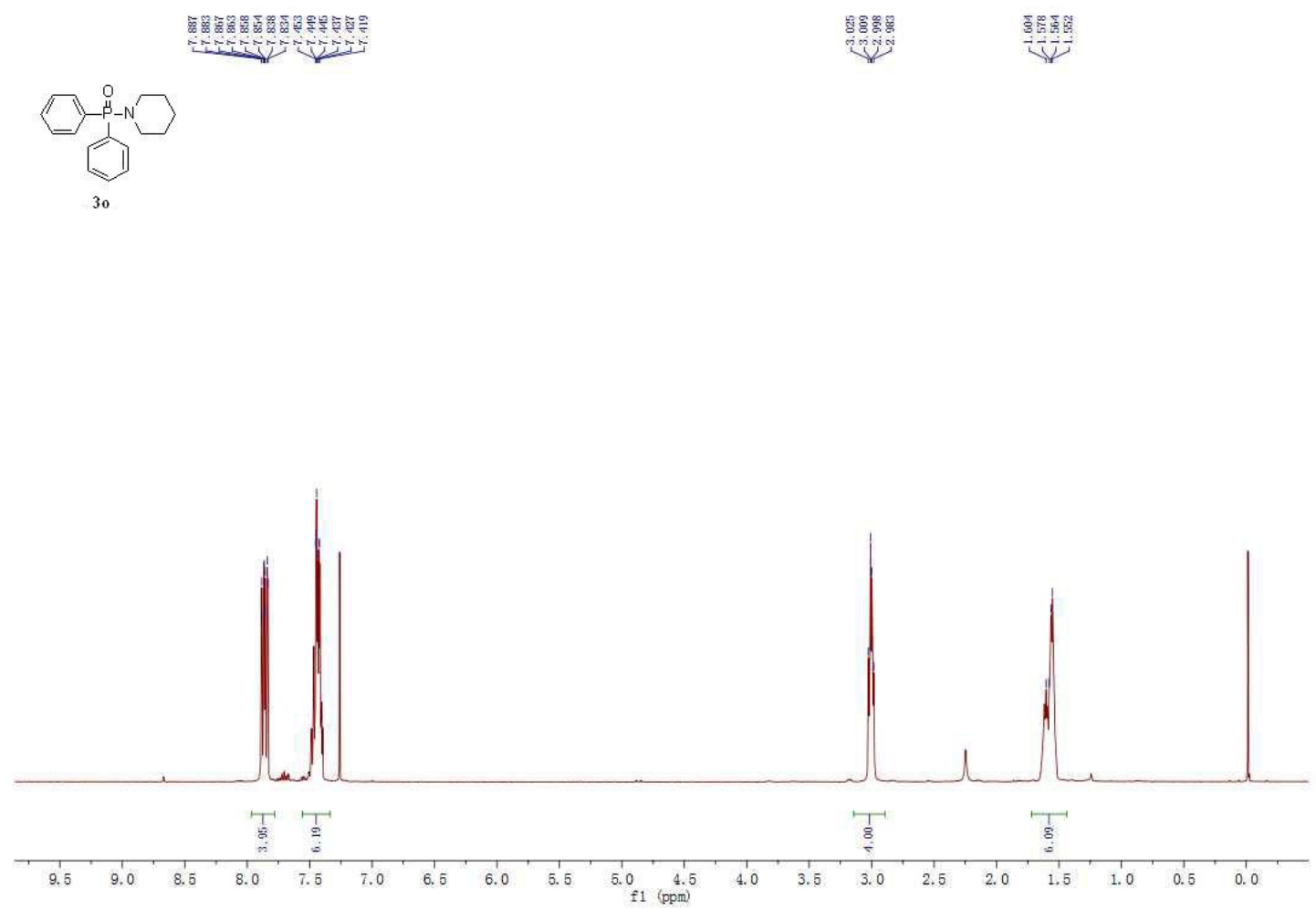
${ }^{13} \mathrm{C}$ NMR of 30

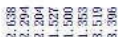

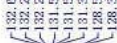

픈

$[1$

30
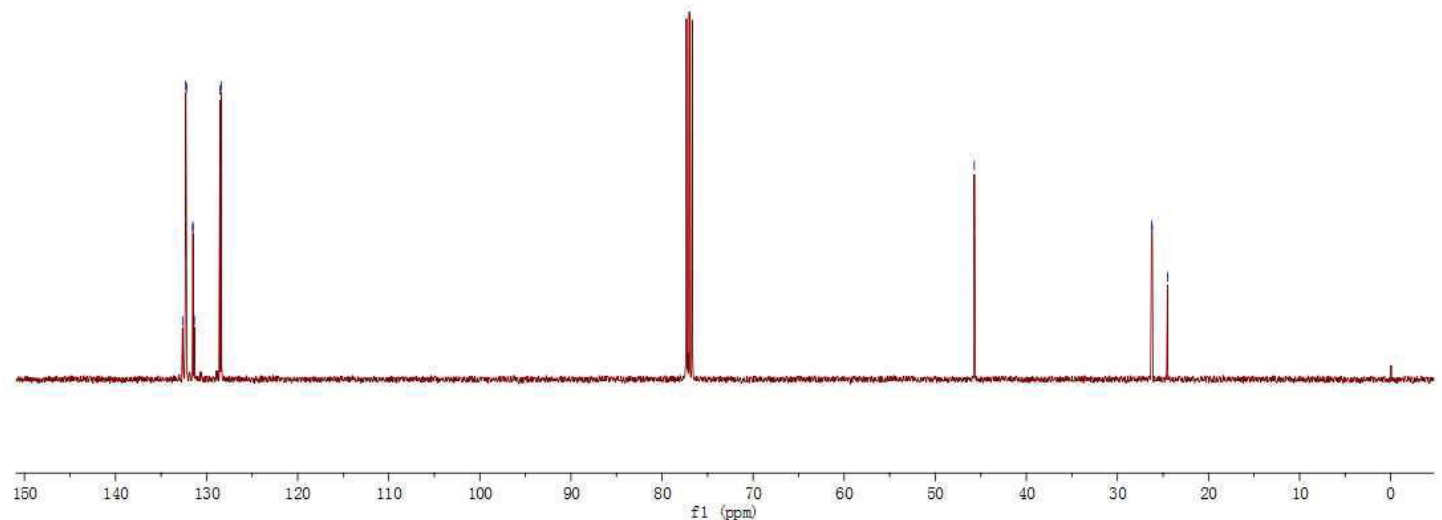

${ }^{31} \mathrm{P}$ NMR of $\mathbf{3 o}$
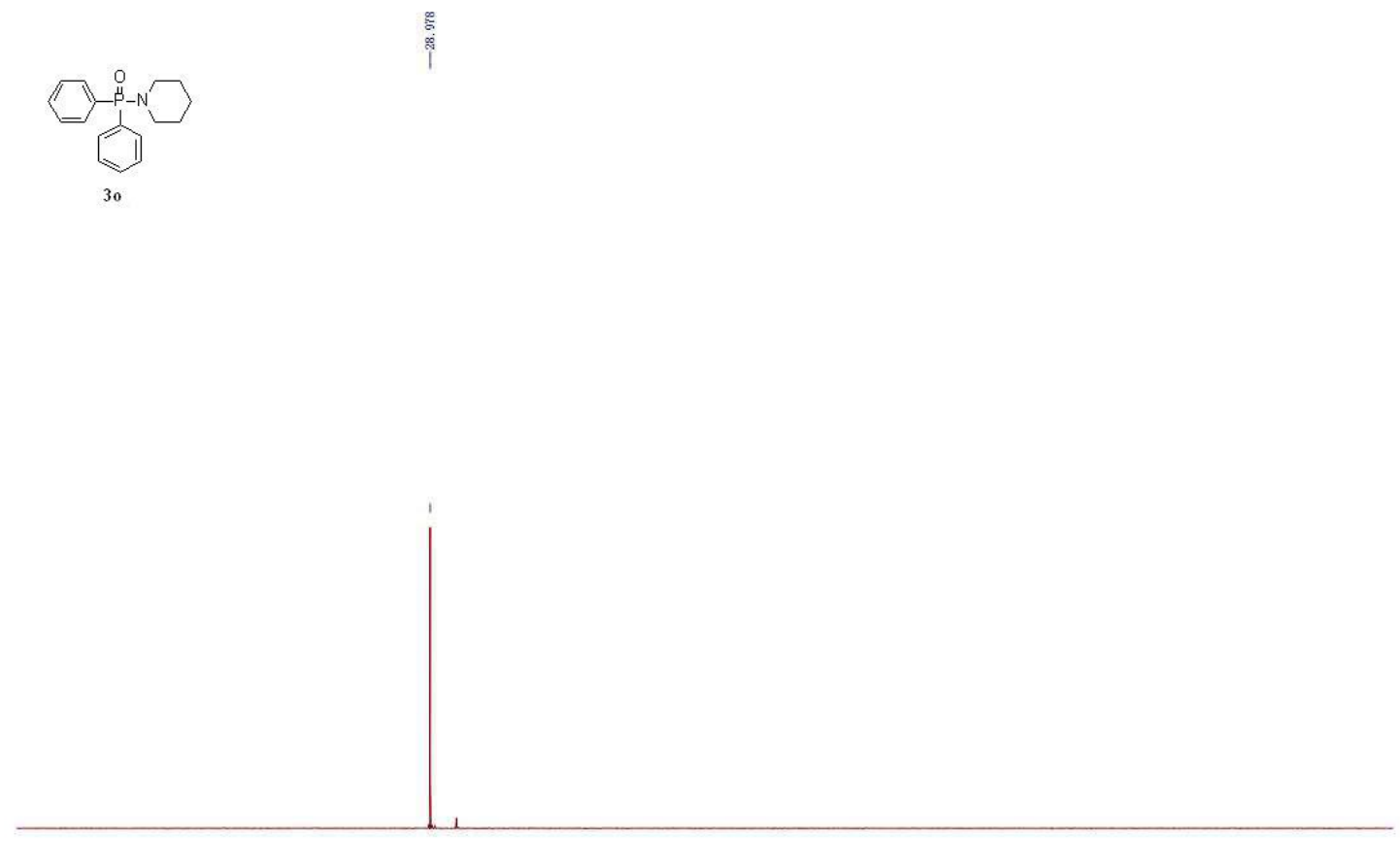

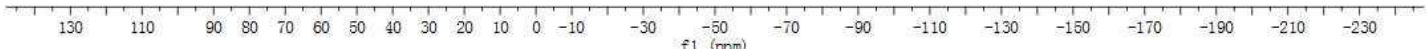


${ }^{1} \mathrm{H}$ NMR of $\mathbf{3 p}$

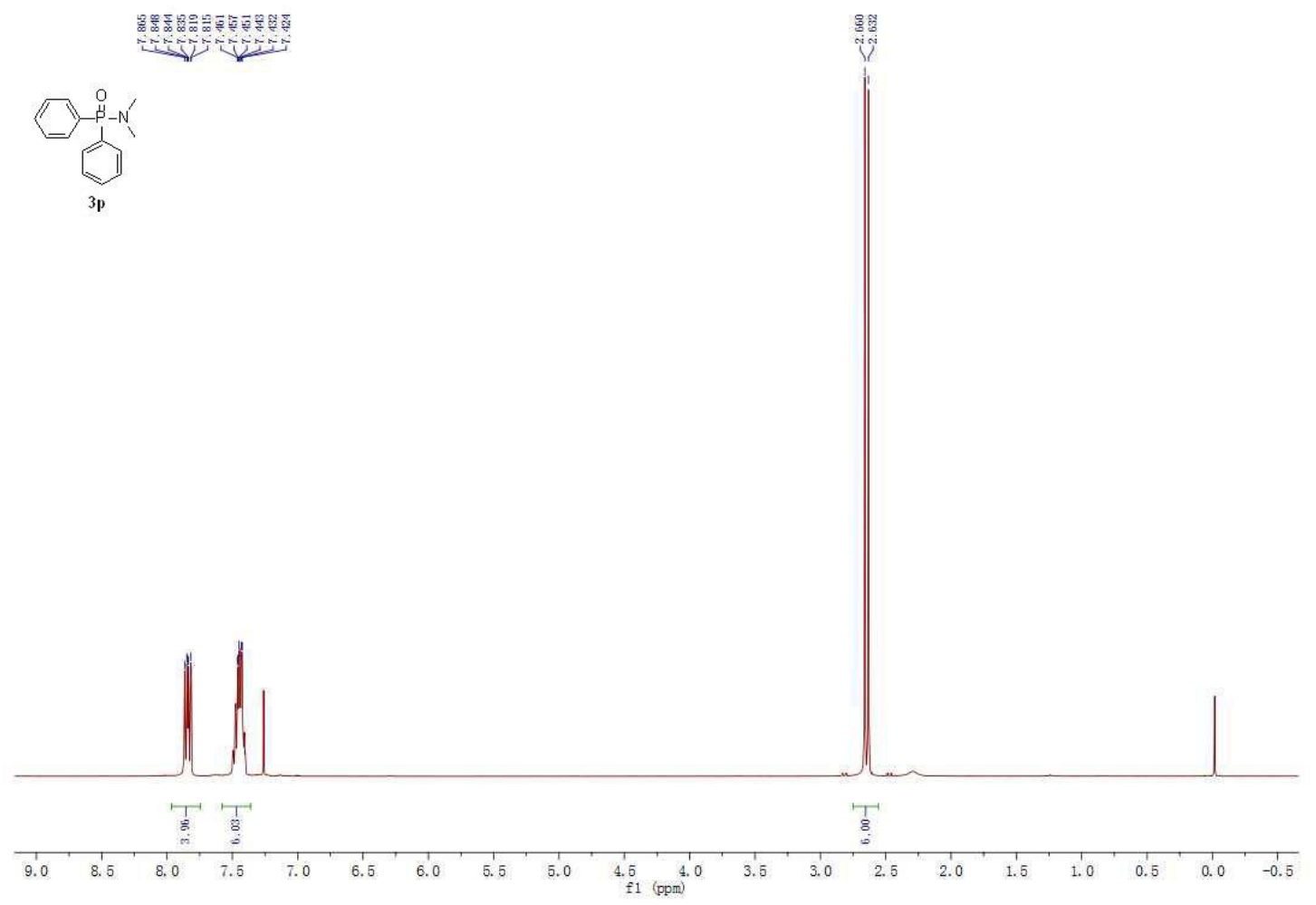

${ }^{13} \mathrm{C}$ NMR of $\mathbf{3 p}$
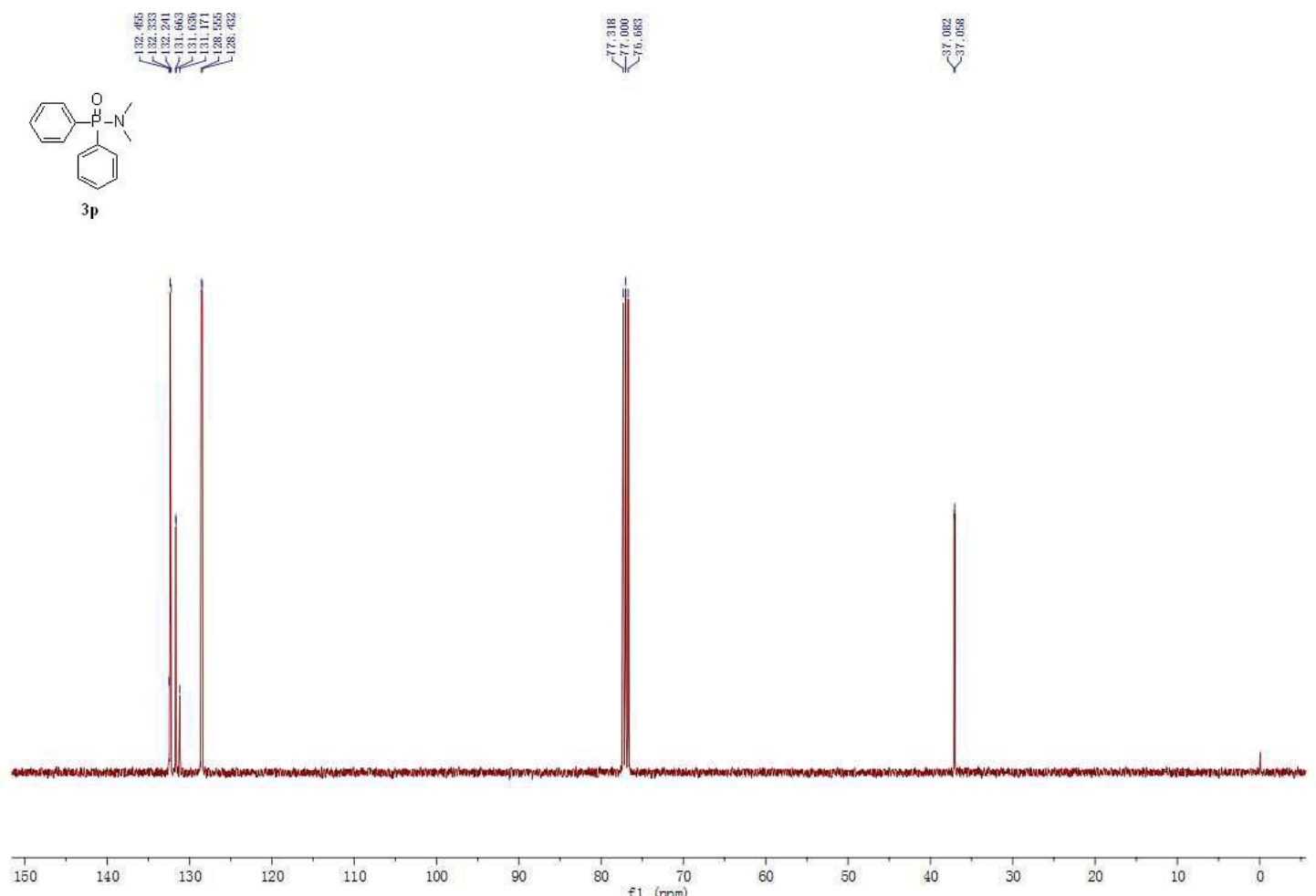
${ }^{31} \mathrm{P}$ NMR of $\mathbf{3 p}$<smiles>CN(C)[PH](=O)(c1ccccc1)c1ccccc1</smiles>

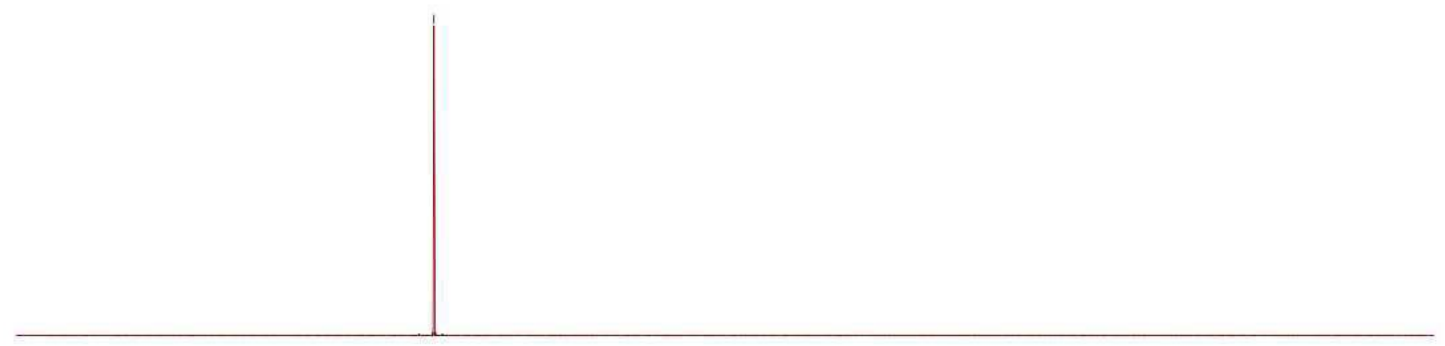

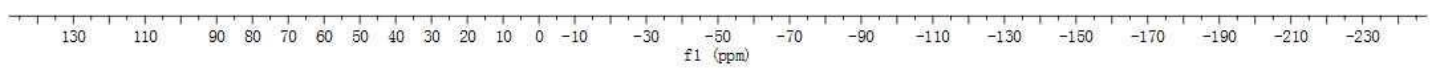

${ }^{1} \mathrm{H}$ NMR of $\mathbf{3 q}$

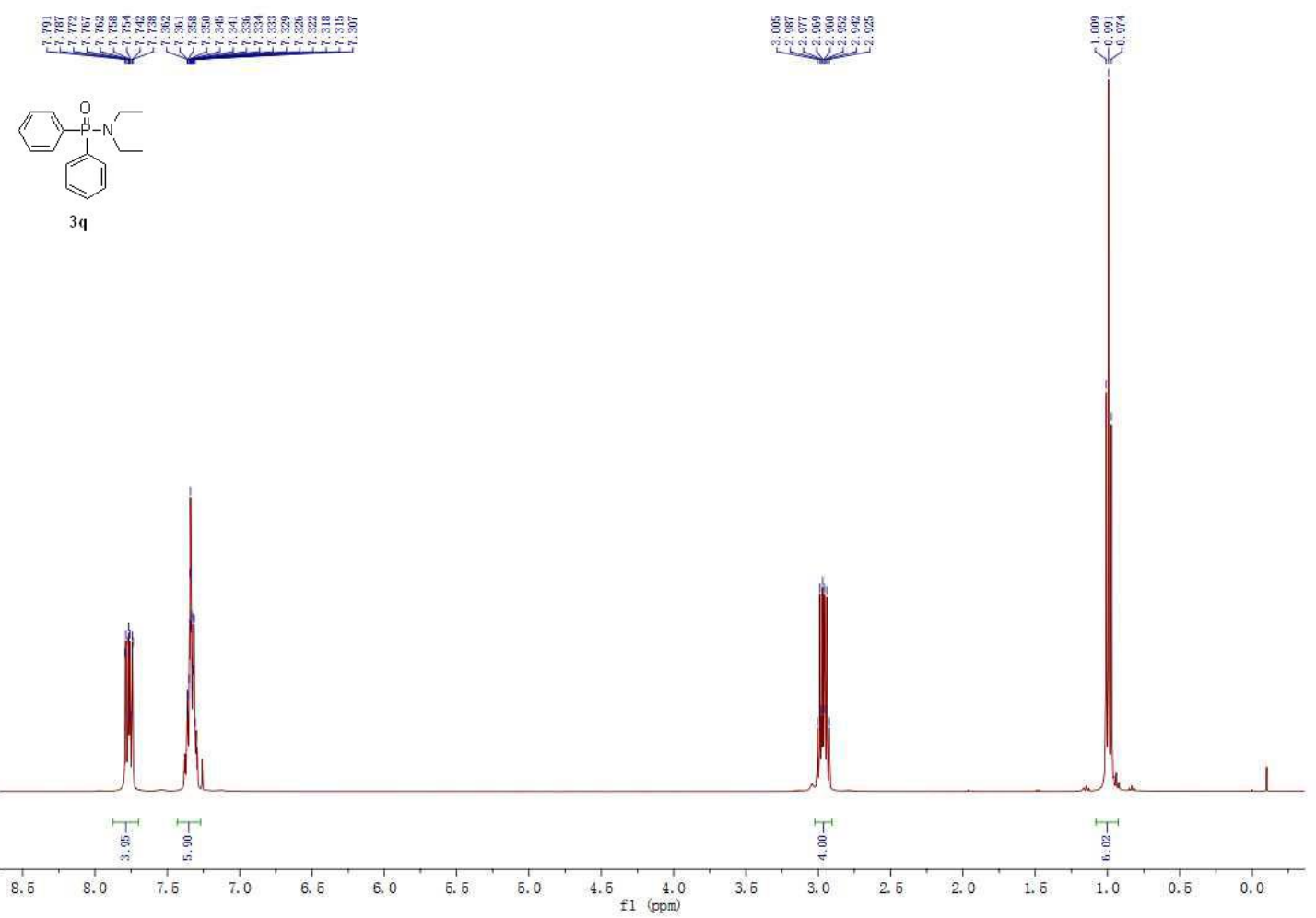


${ }^{13} \mathrm{C}$ NMR of $\mathbf{3 q}$

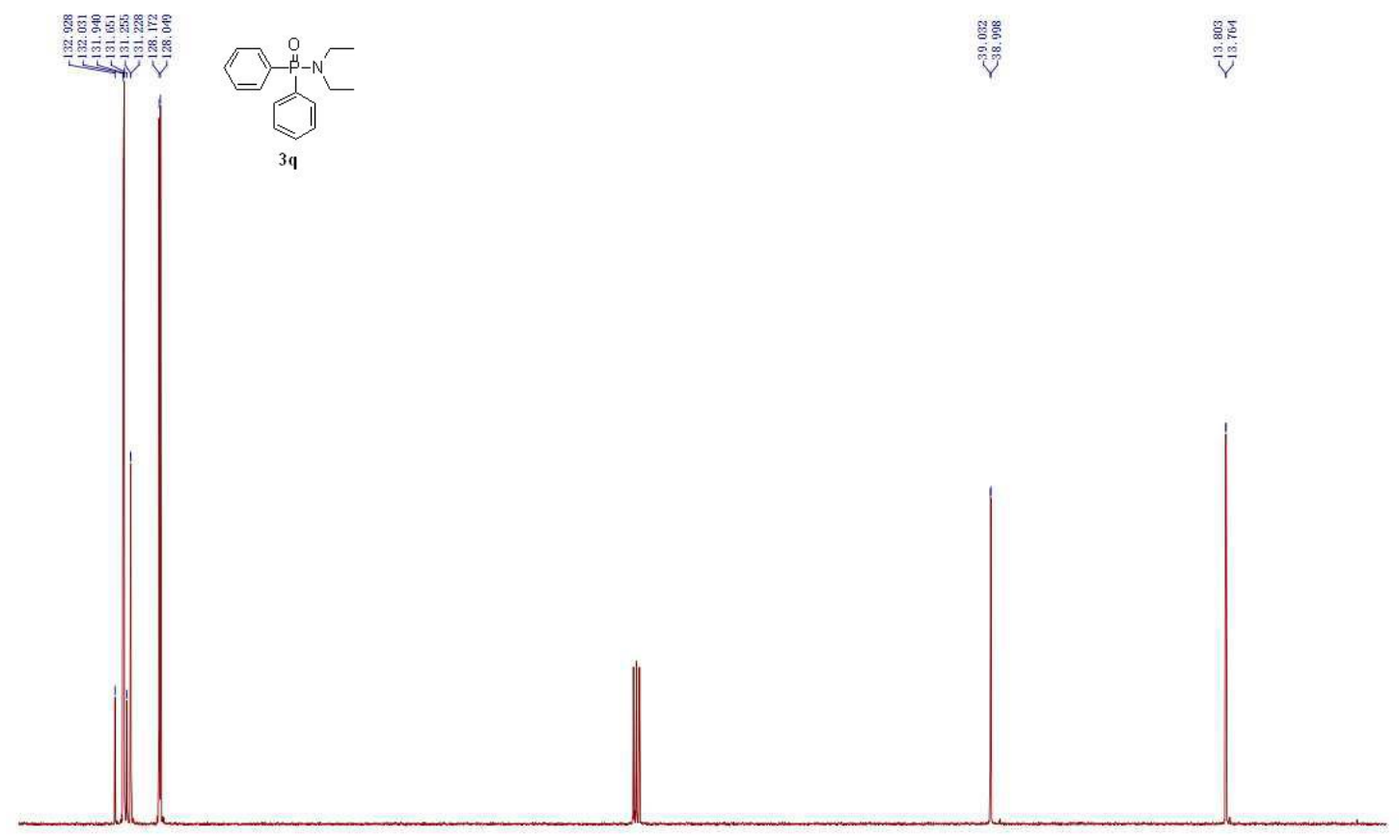

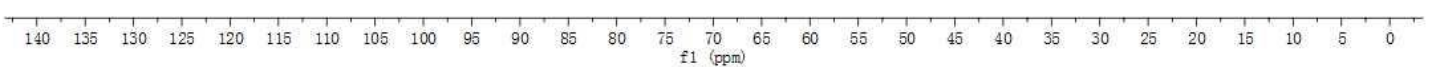

${ }^{31}$ P NMR of $\mathbf{3 q}$
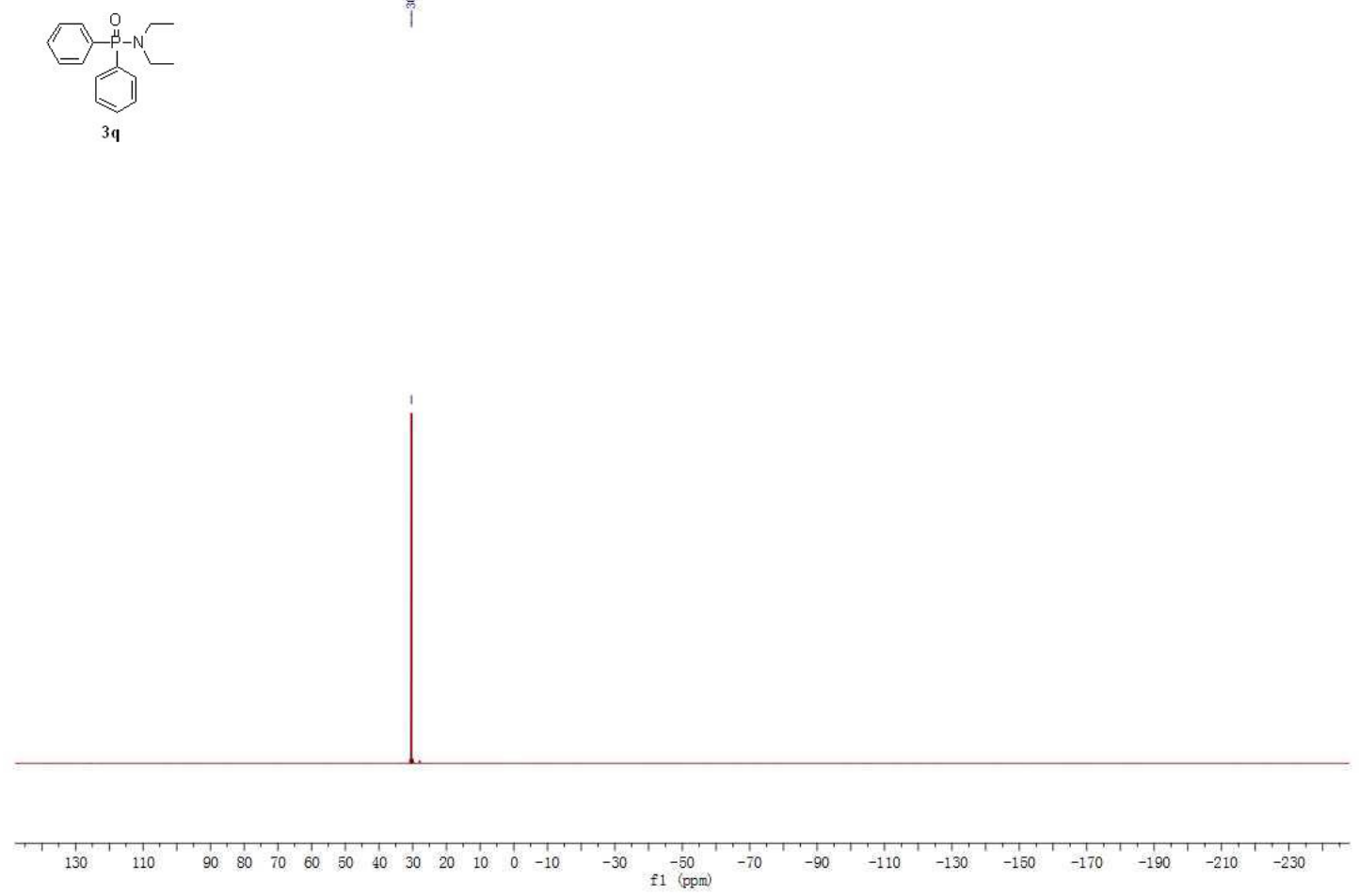


\section{${ }^{1} \mathrm{H}$ NMR of $3 \mathbf{s}$}

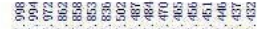

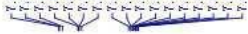

(1)

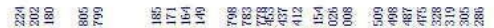

iए

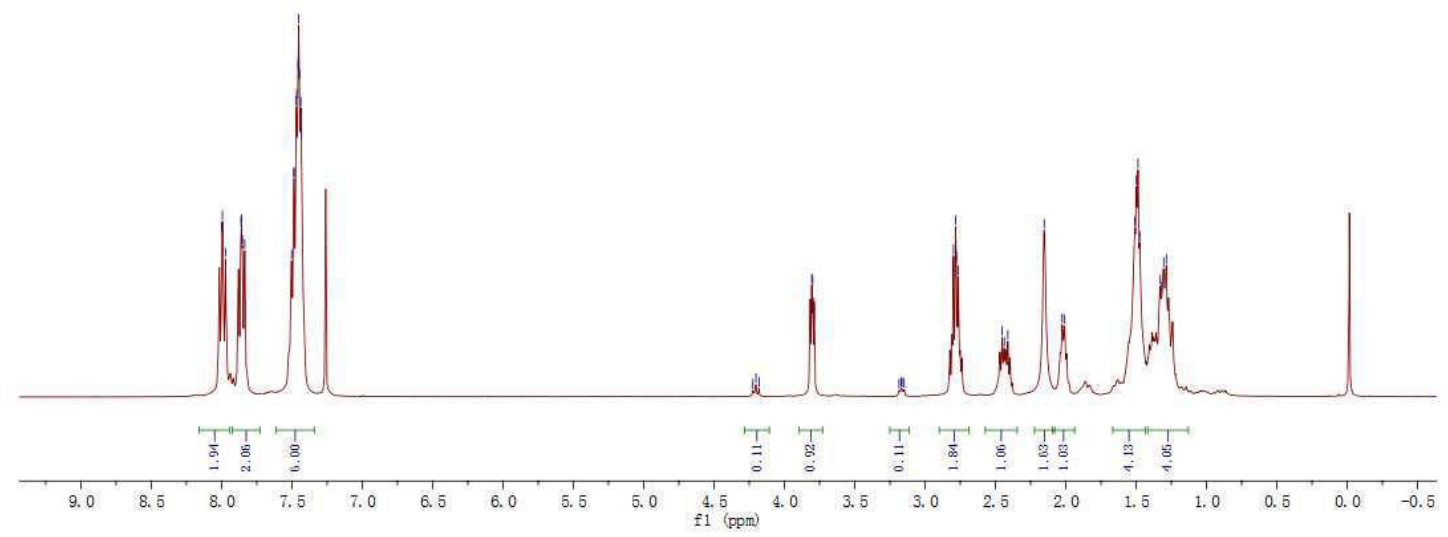

${ }^{13} \mathrm{C}$ NMR of $3 \mathrm{~s}$

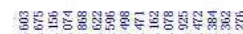

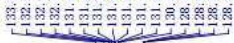<smiles>O=P(c1ccccc1)(c1ccccc1)N1CC2CCCCC2C1</smiles>

$3 \mathrm{~s}$

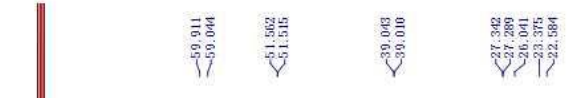

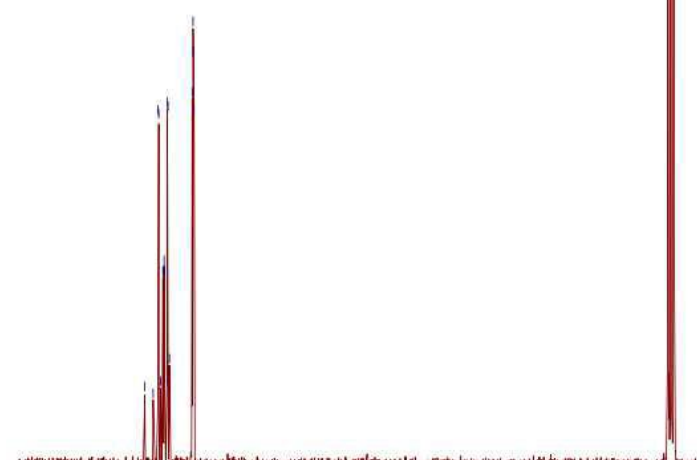

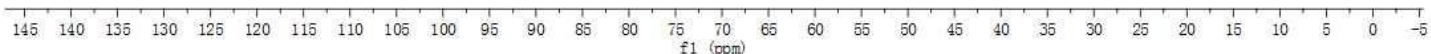


${ }^{31}$ P NMR of $\mathbf{3 s}$
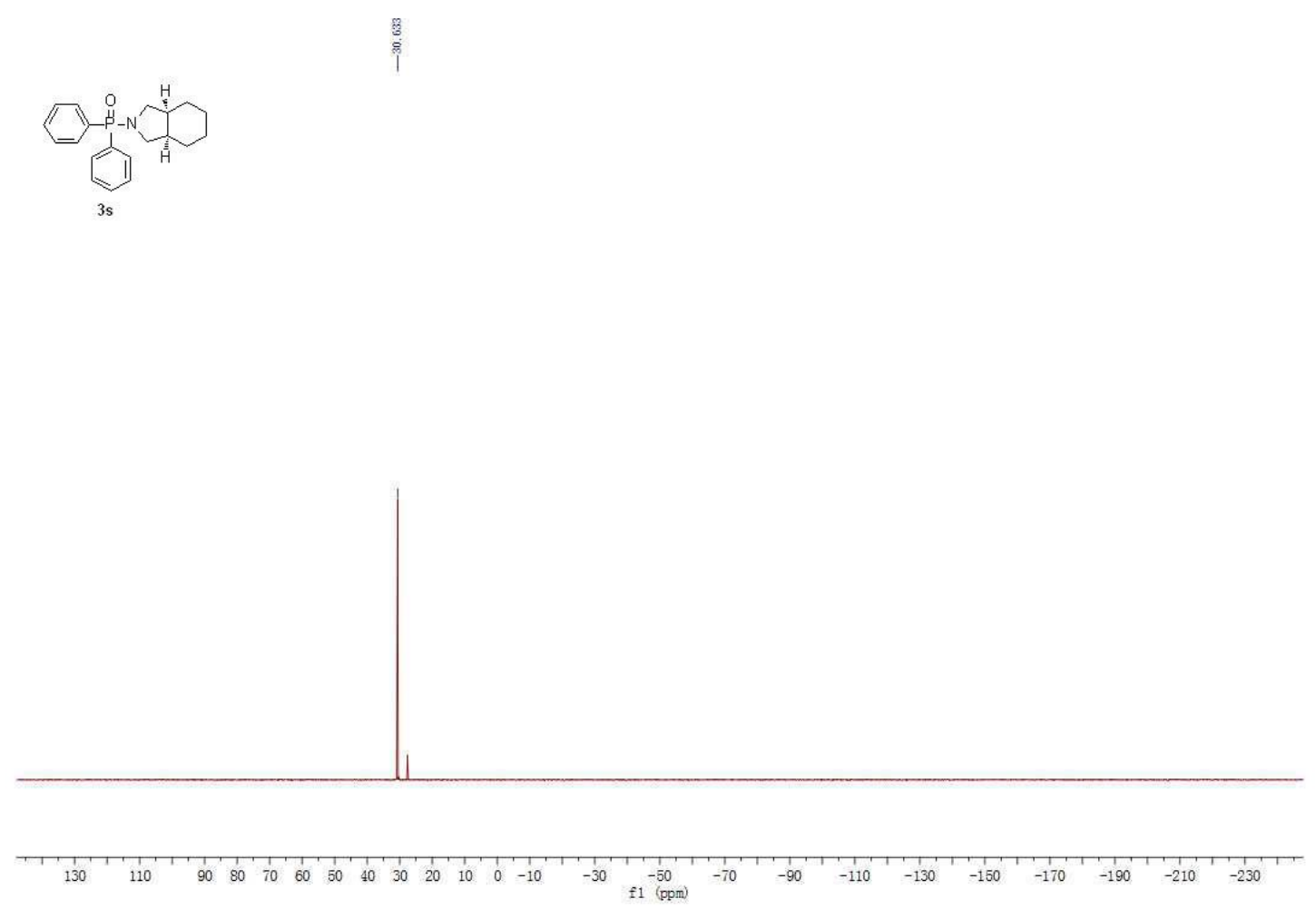

${ }^{1} \mathrm{H}$ NMR of $\mathbf{3 t}$

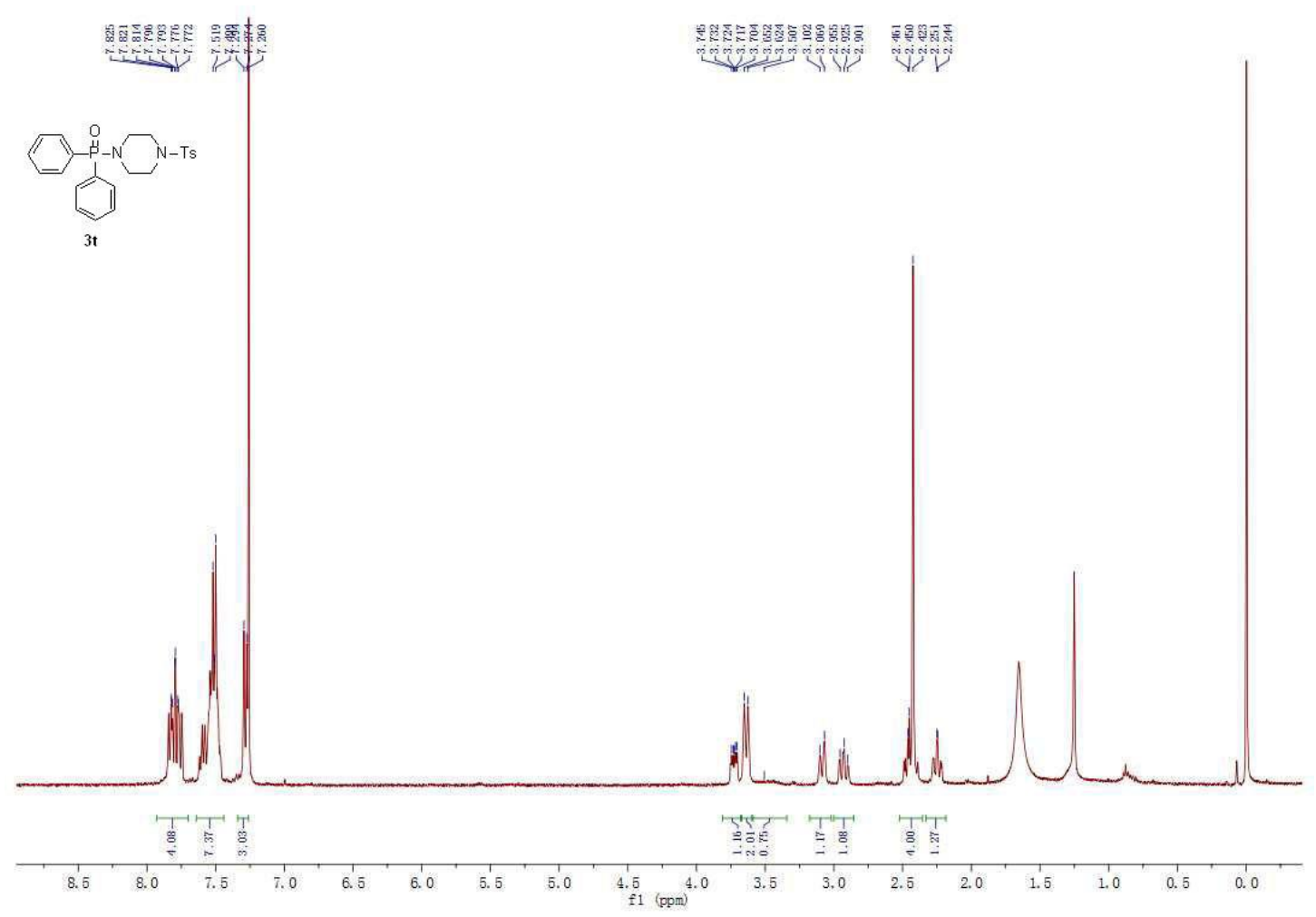


${ }^{13} \mathrm{C}$ NMR of $\mathbf{3 t}$
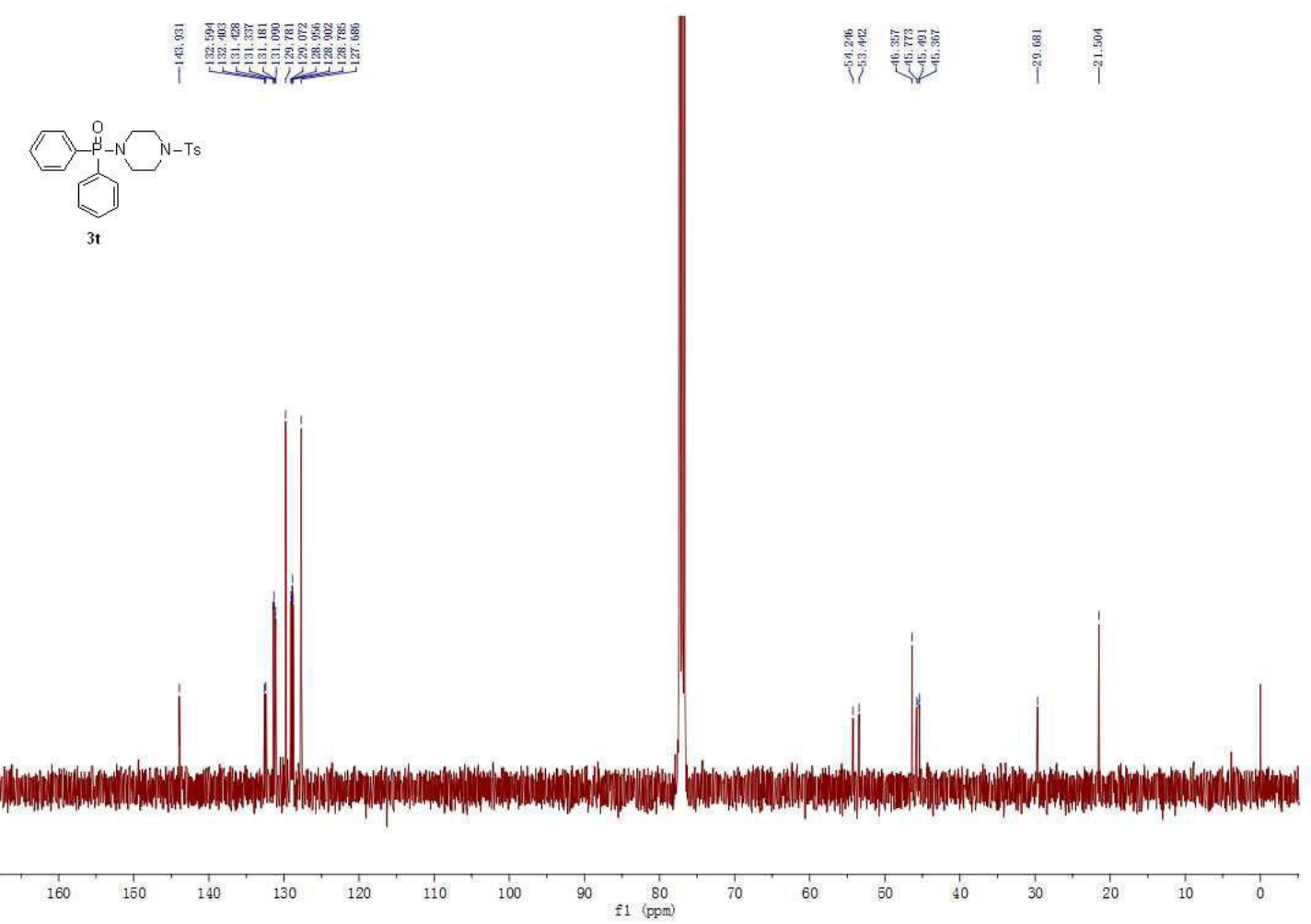

${ }^{31} \mathrm{P}$ NMR of $\mathbf{3 t}$
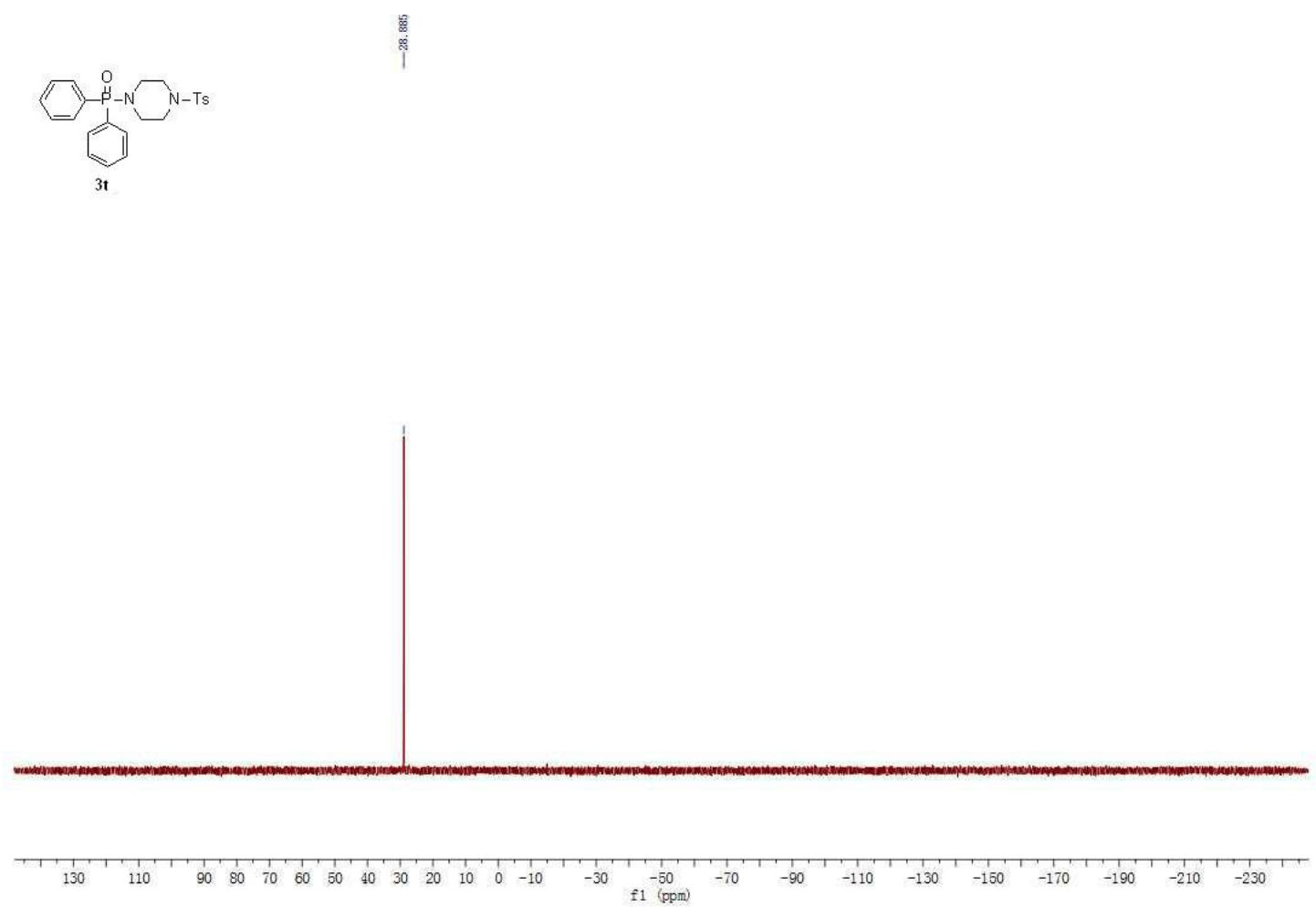


\section{${ }^{1} \mathrm{H}$ NMR of $\mathbf{3 u}$}

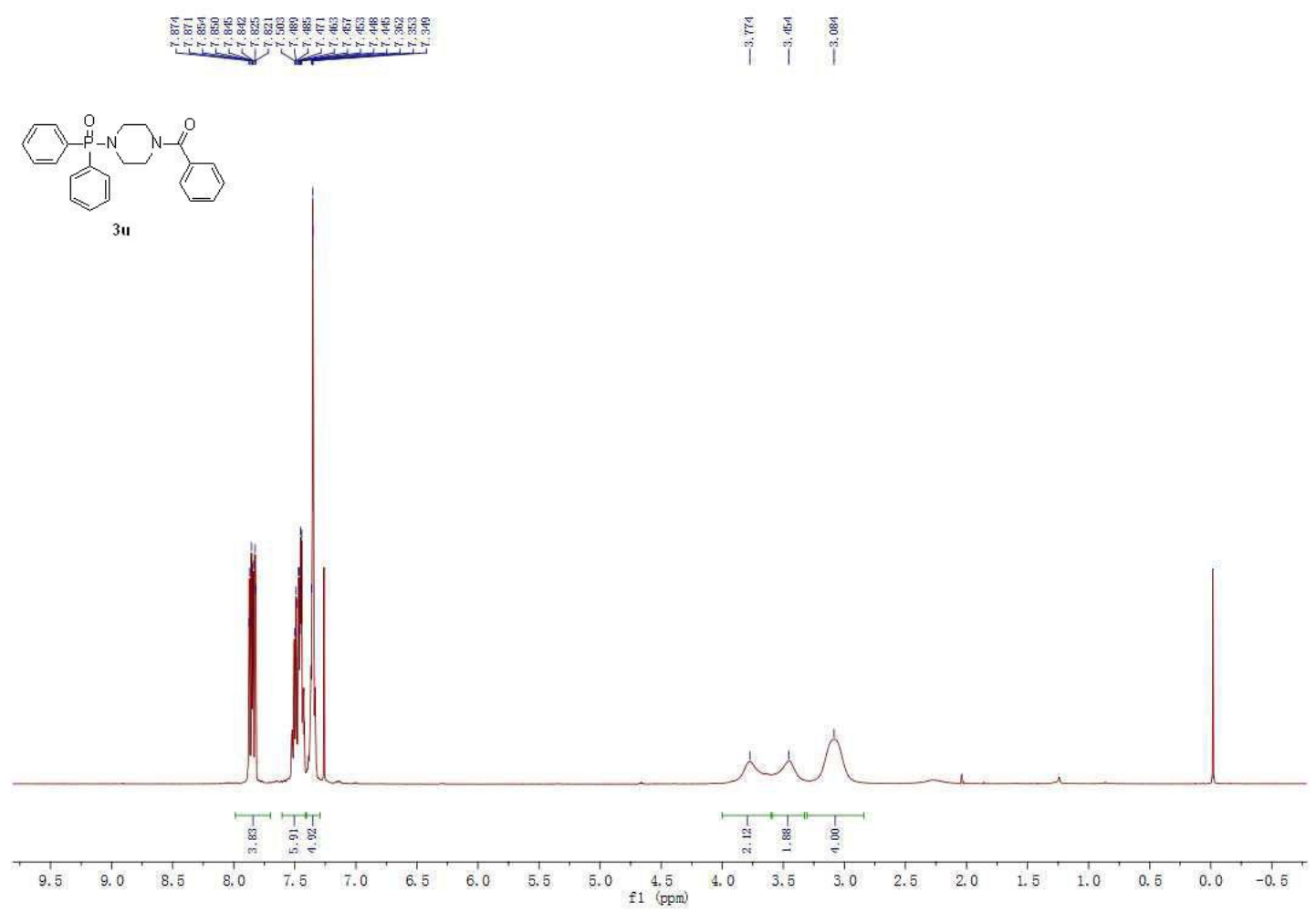

${ }^{13} \mathrm{C}$ NMR of $\mathbf{3 u}$

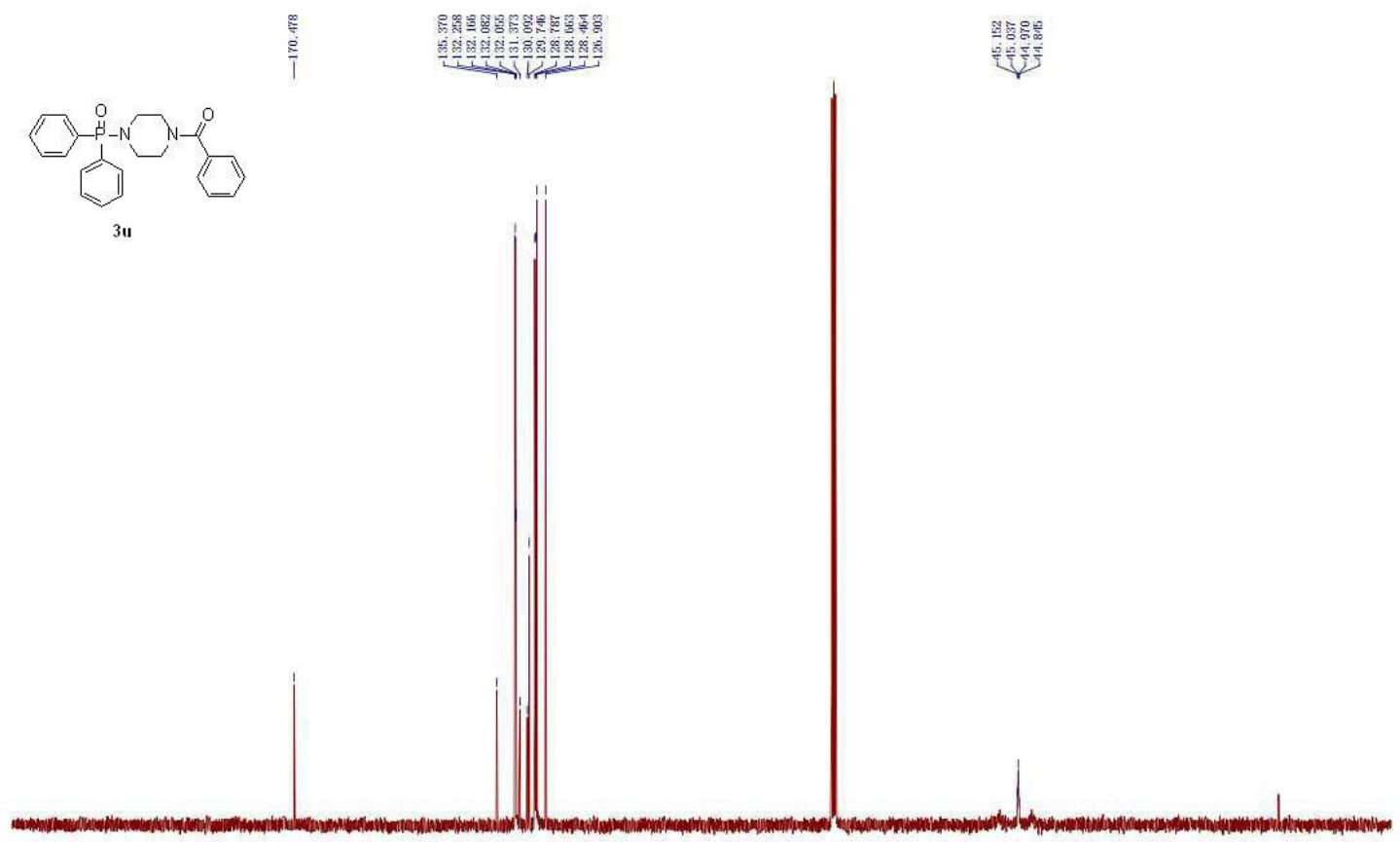

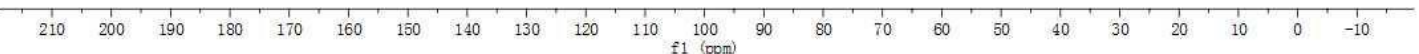


${ }^{31}$ P NMR of 3u

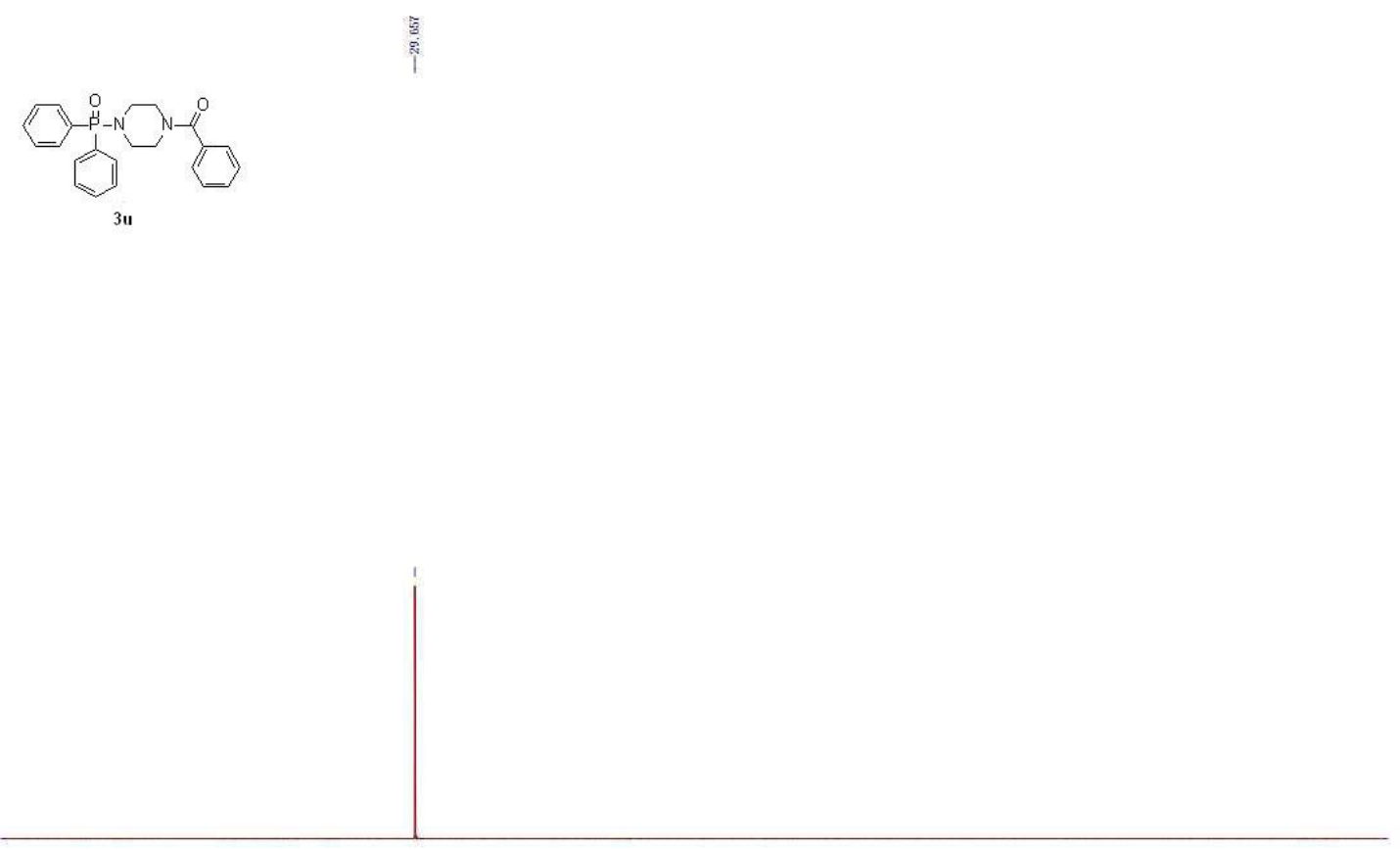

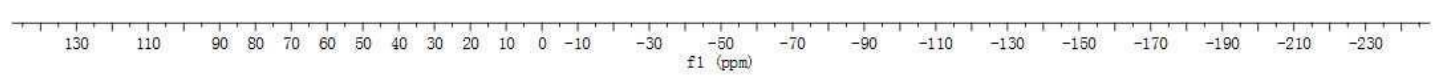

${ }^{1} \mathrm{H}$ NMR of $\mathbf{3 v}$

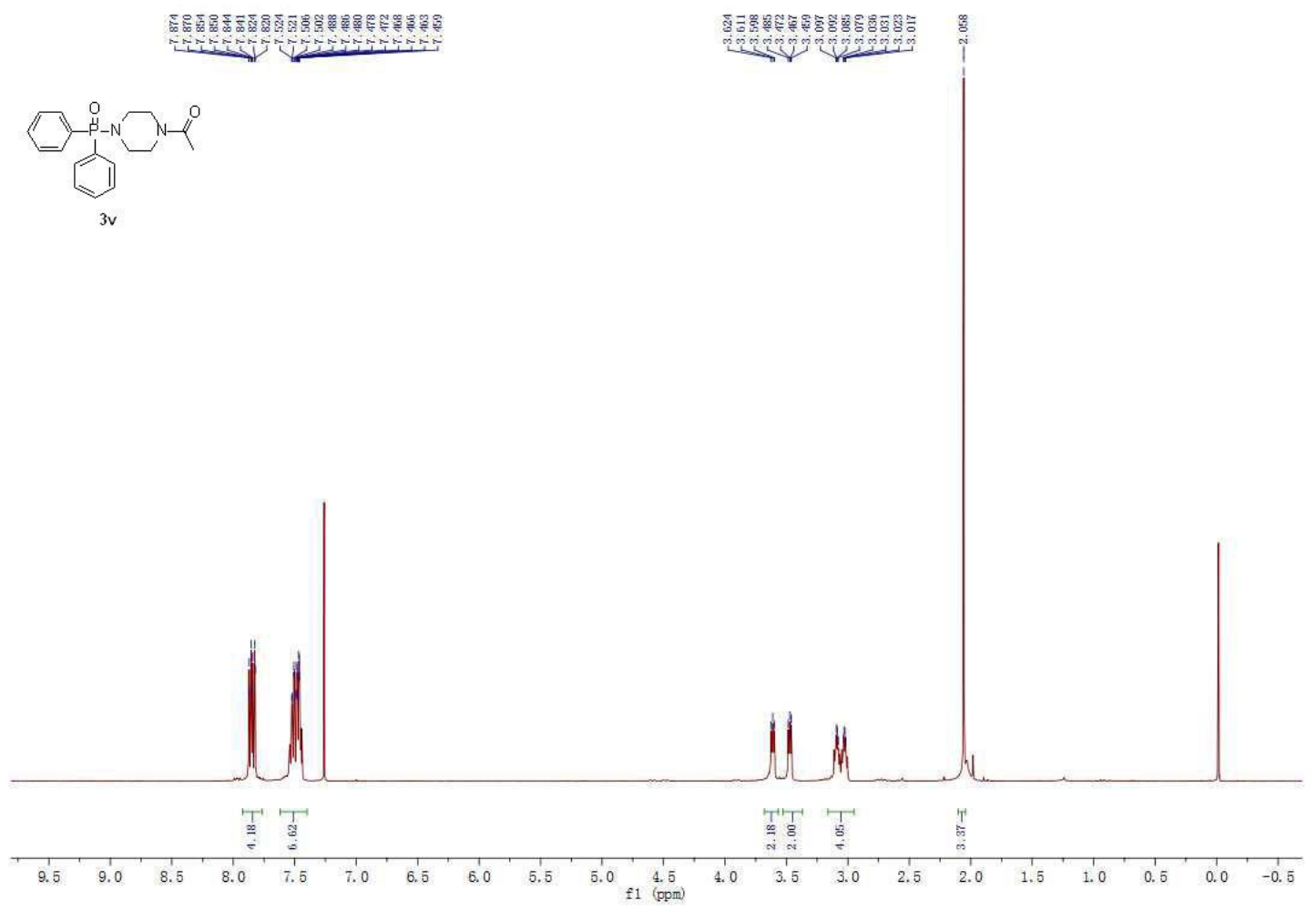


${ }^{13} \mathrm{C}$ NMR of 3v
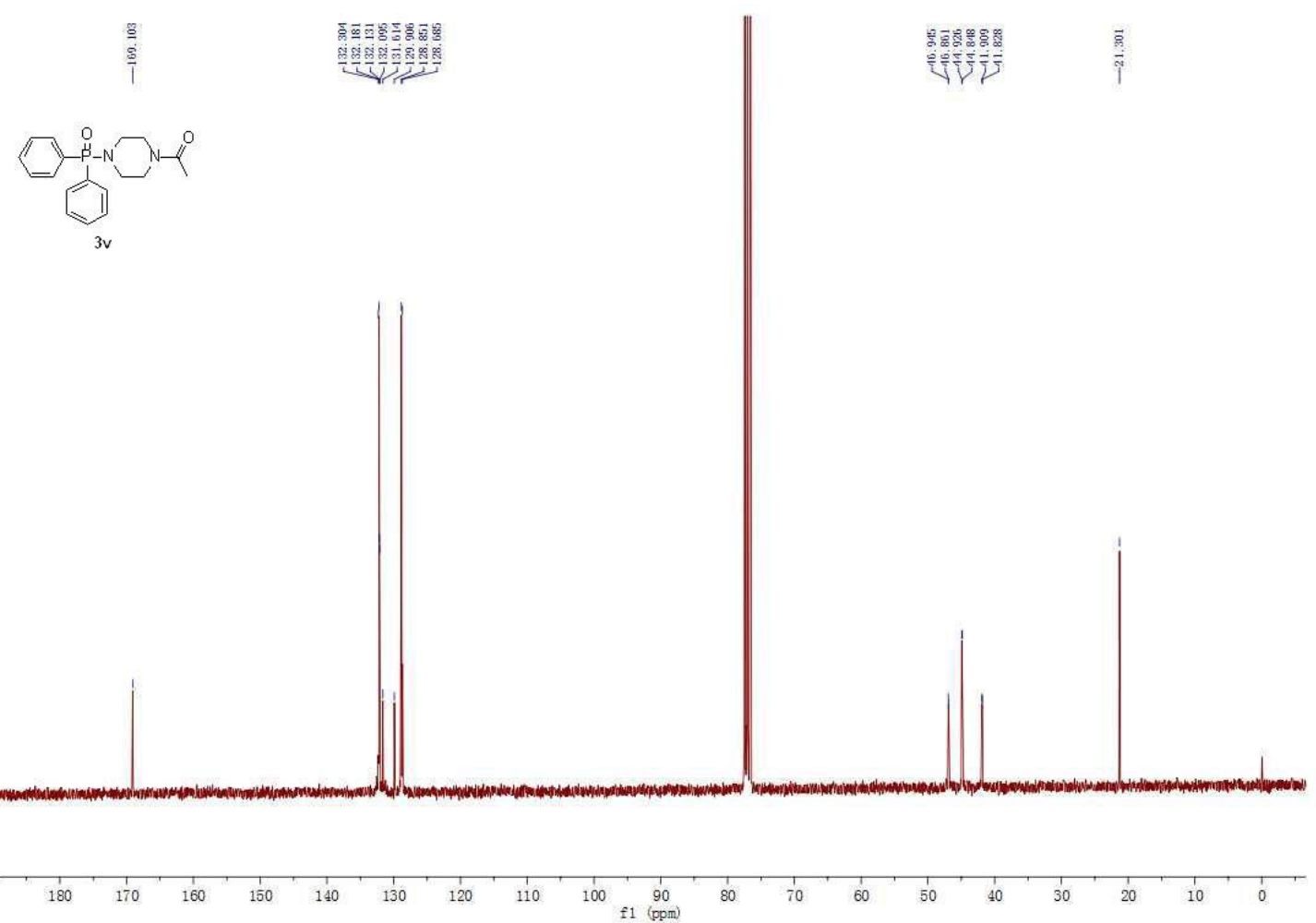

${ }^{31} \mathrm{P}$ NMR of $\mathbf{3 v}$
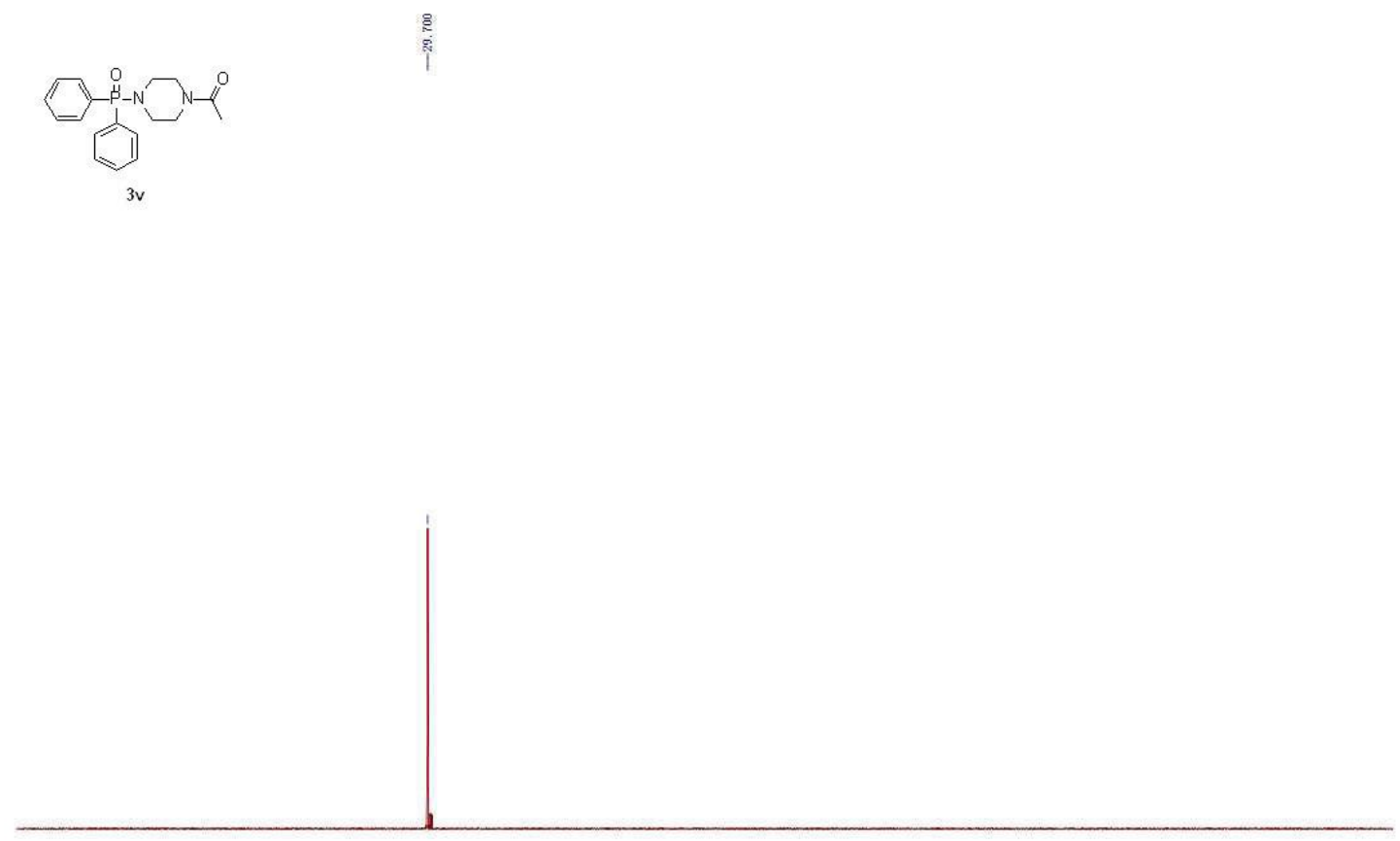

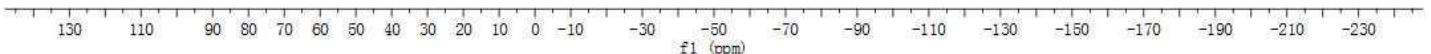


${ }^{1} \mathrm{H}$ NMR of $\mathbf{3 w}$
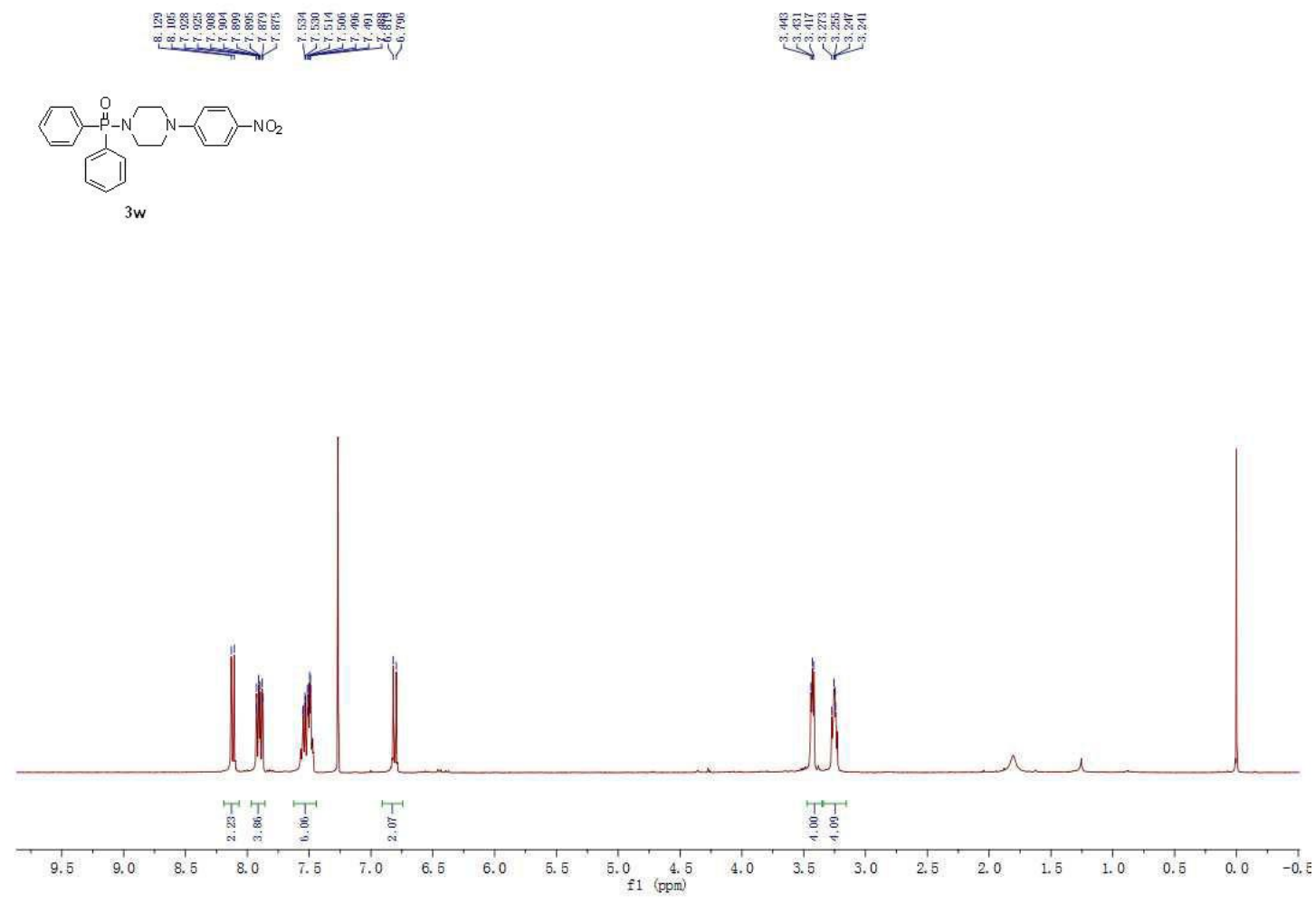

${ }^{13} \mathrm{C}$ NMR of $\mathbf{3 w}$

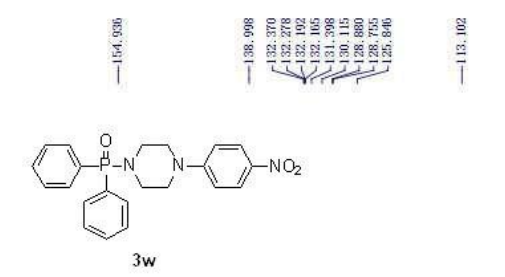

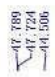
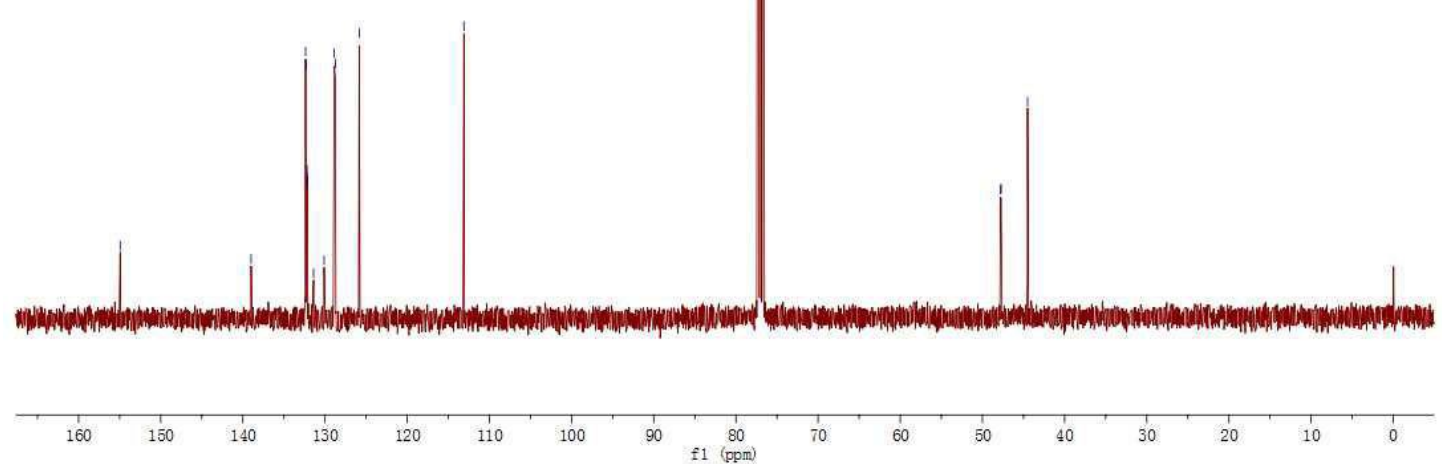
${ }^{31} \mathrm{P}$ NMR of $\mathbf{3 w}$

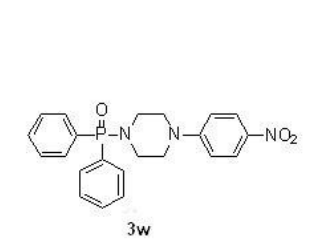

$\stackrel{8}{8}$

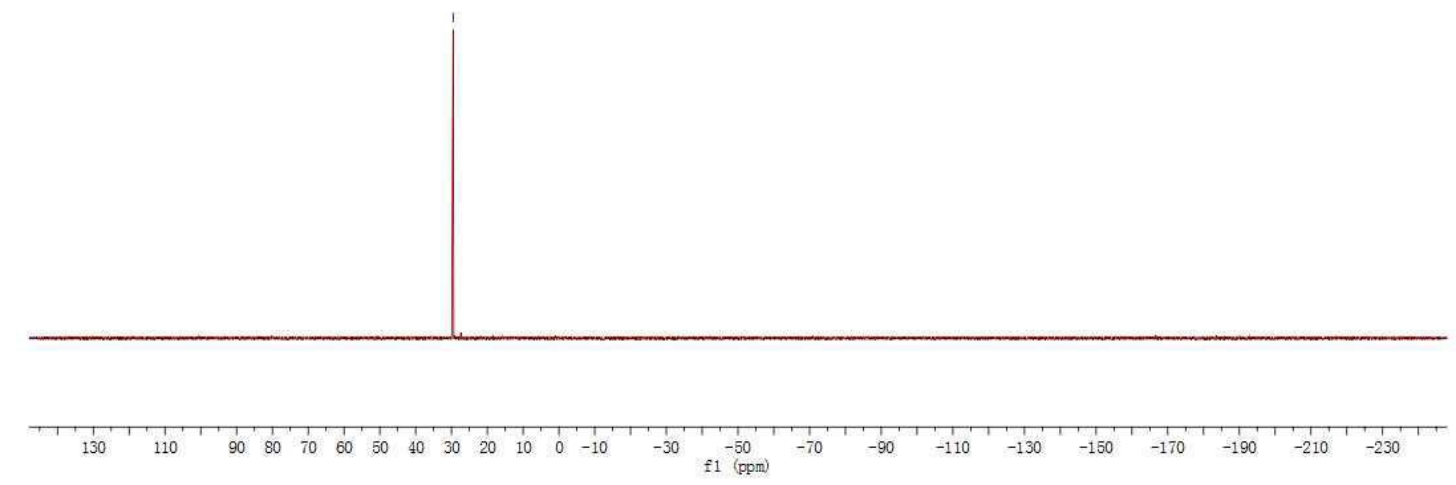

${ }^{1} \mathrm{H}$ NMR of $\mathbf{3 x}$
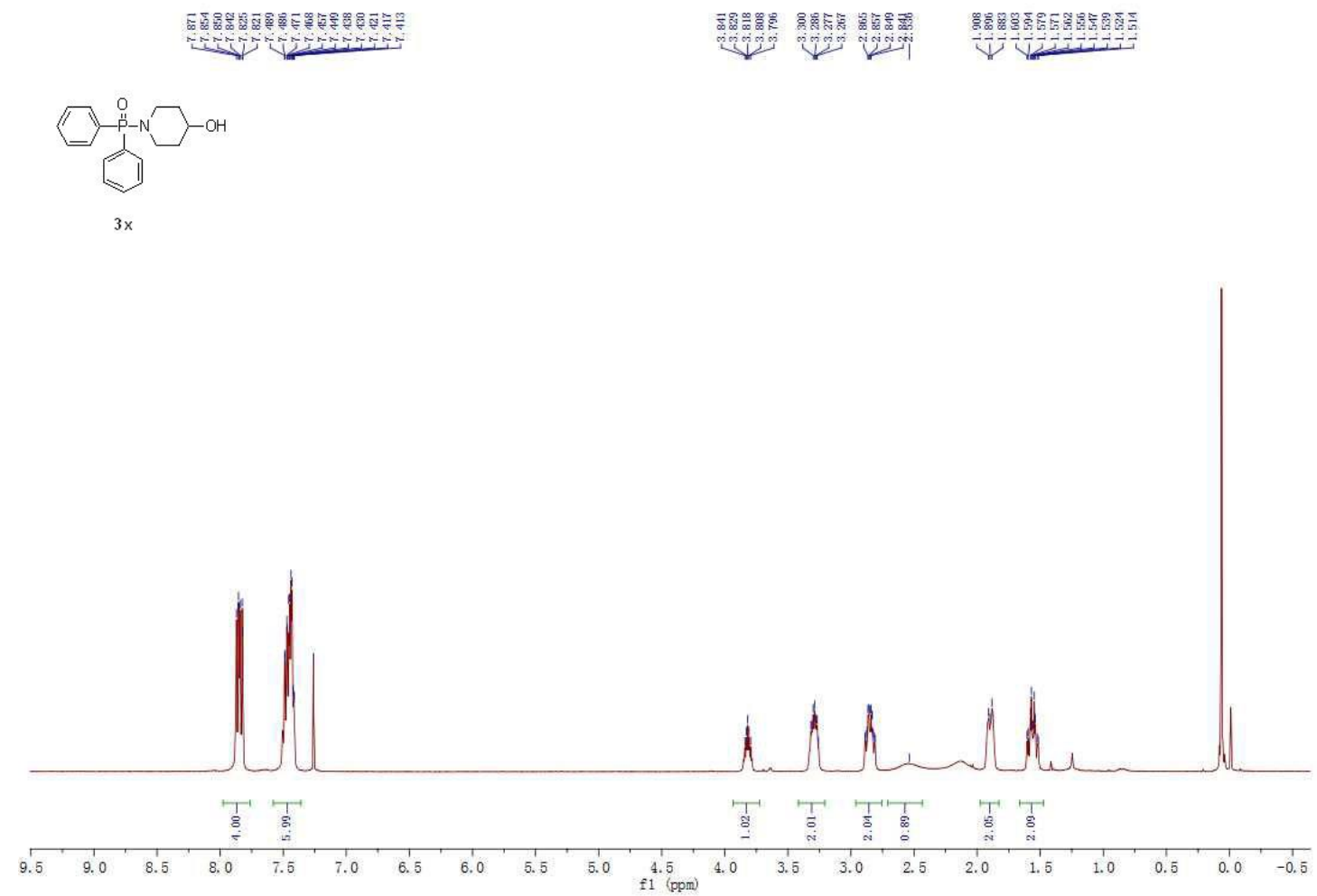
${ }^{13} \mathrm{C}$ NMR of $\mathbf{3 x}$

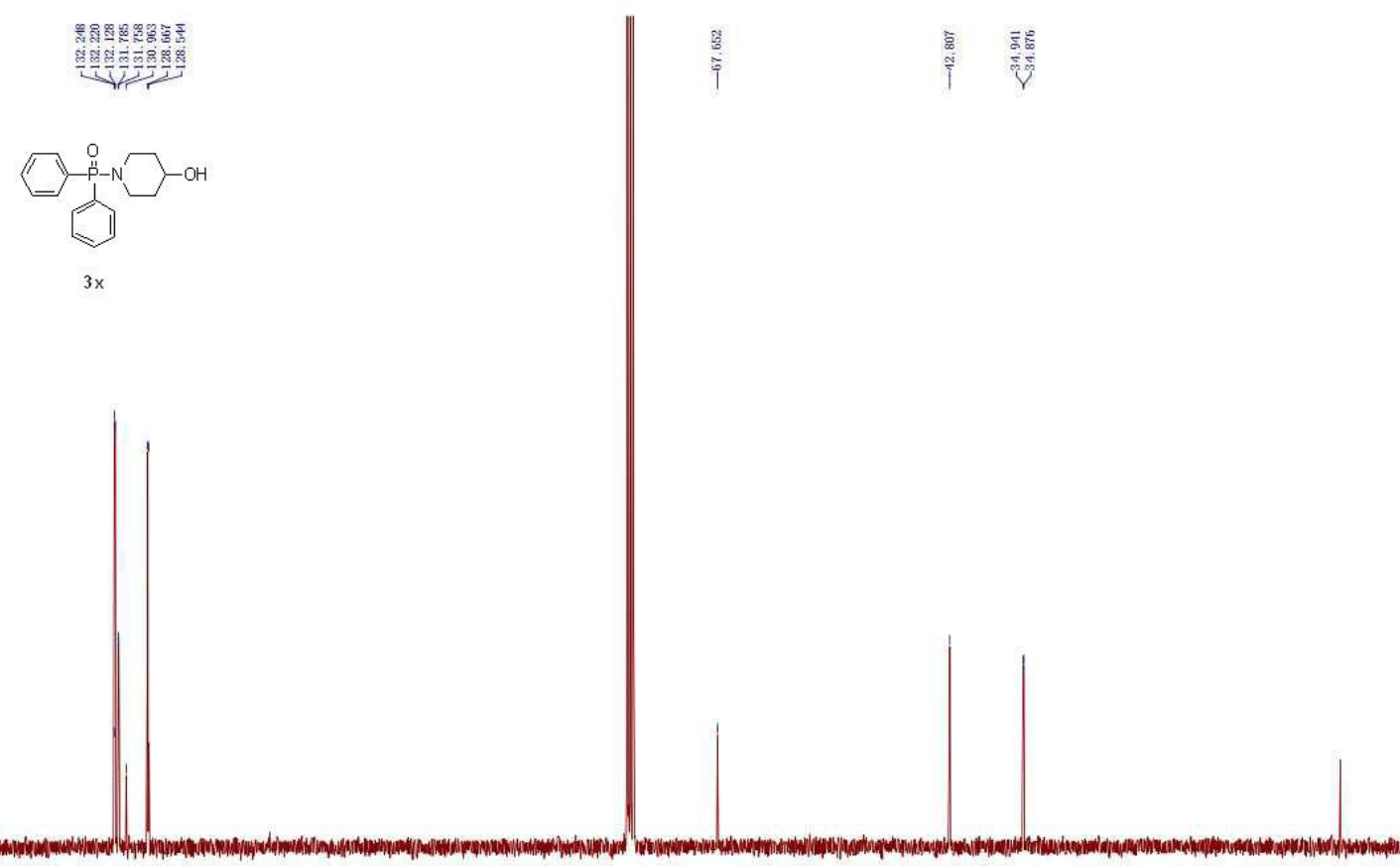

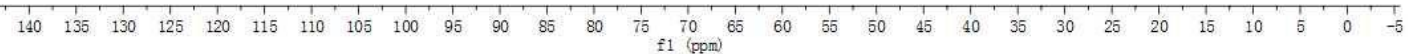

${ }^{31} \mathrm{P}$ NMR of $\mathbf{3 x}$

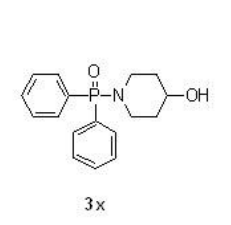

$\sum_{i}$

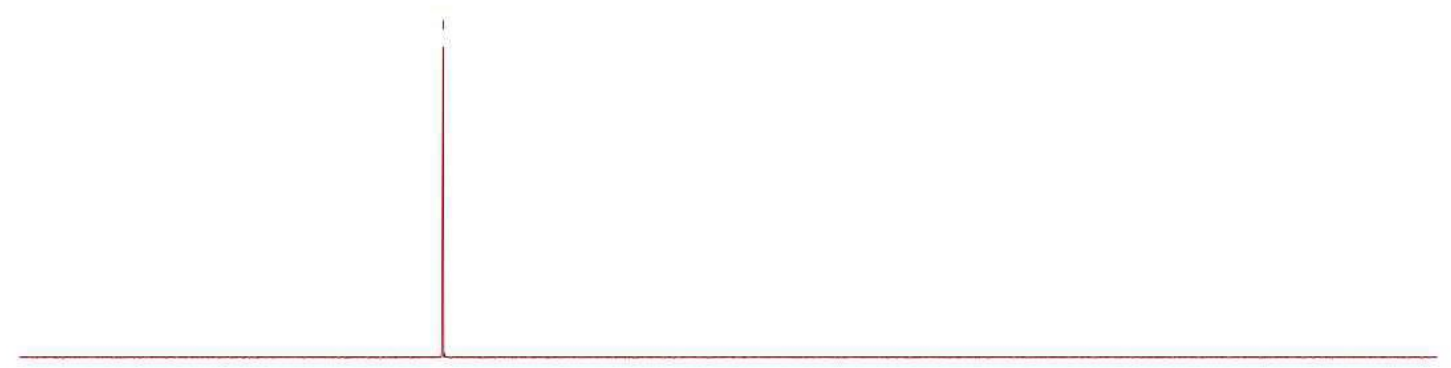

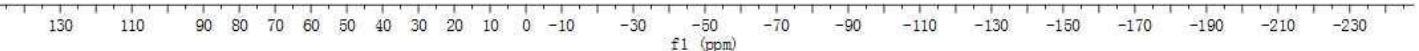


${ }^{1} \mathrm{H}$ NMR of $\mathbf{3 y}$

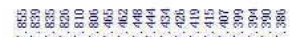

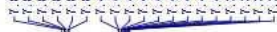

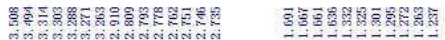

ving<smiles>CC1CCCCC12CCCC21CCCCC1</smiles>

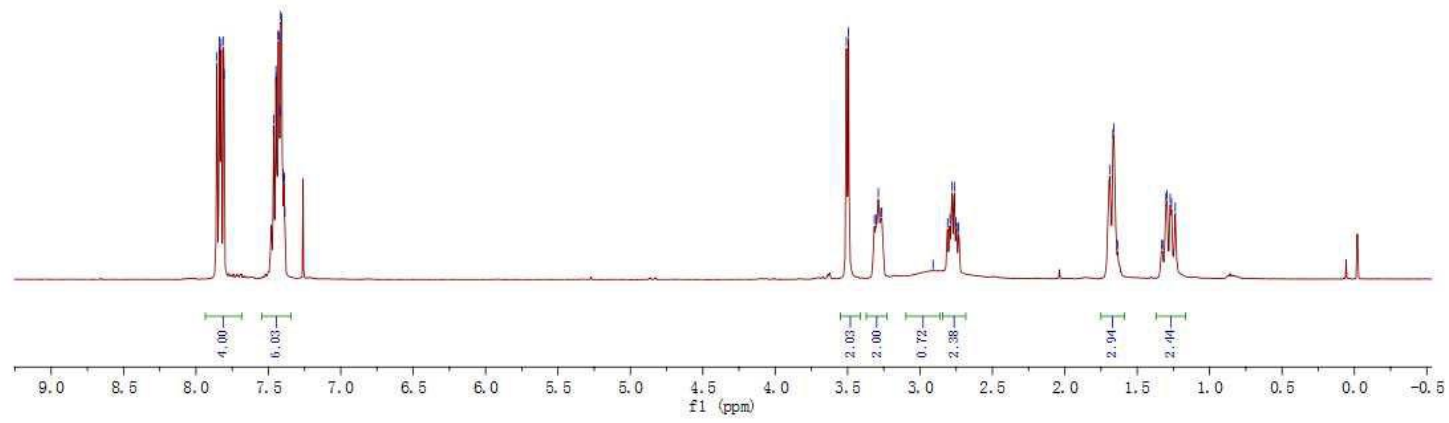

${ }^{13}$ C NMR of $\mathbf{3 y}$
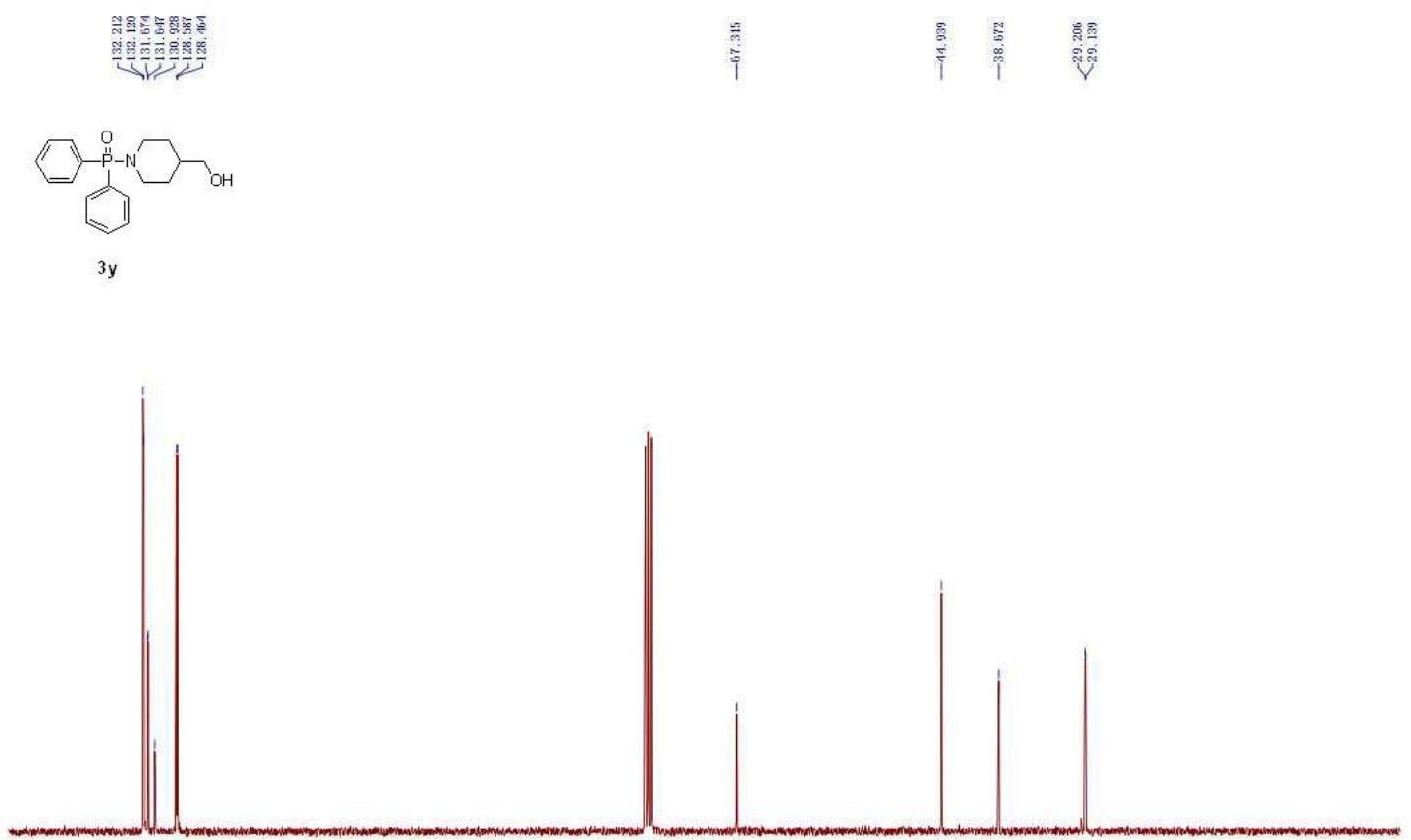

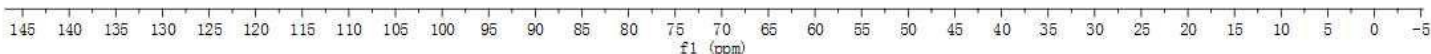


${ }^{31}$ P NMR of $\mathbf{3 y}$
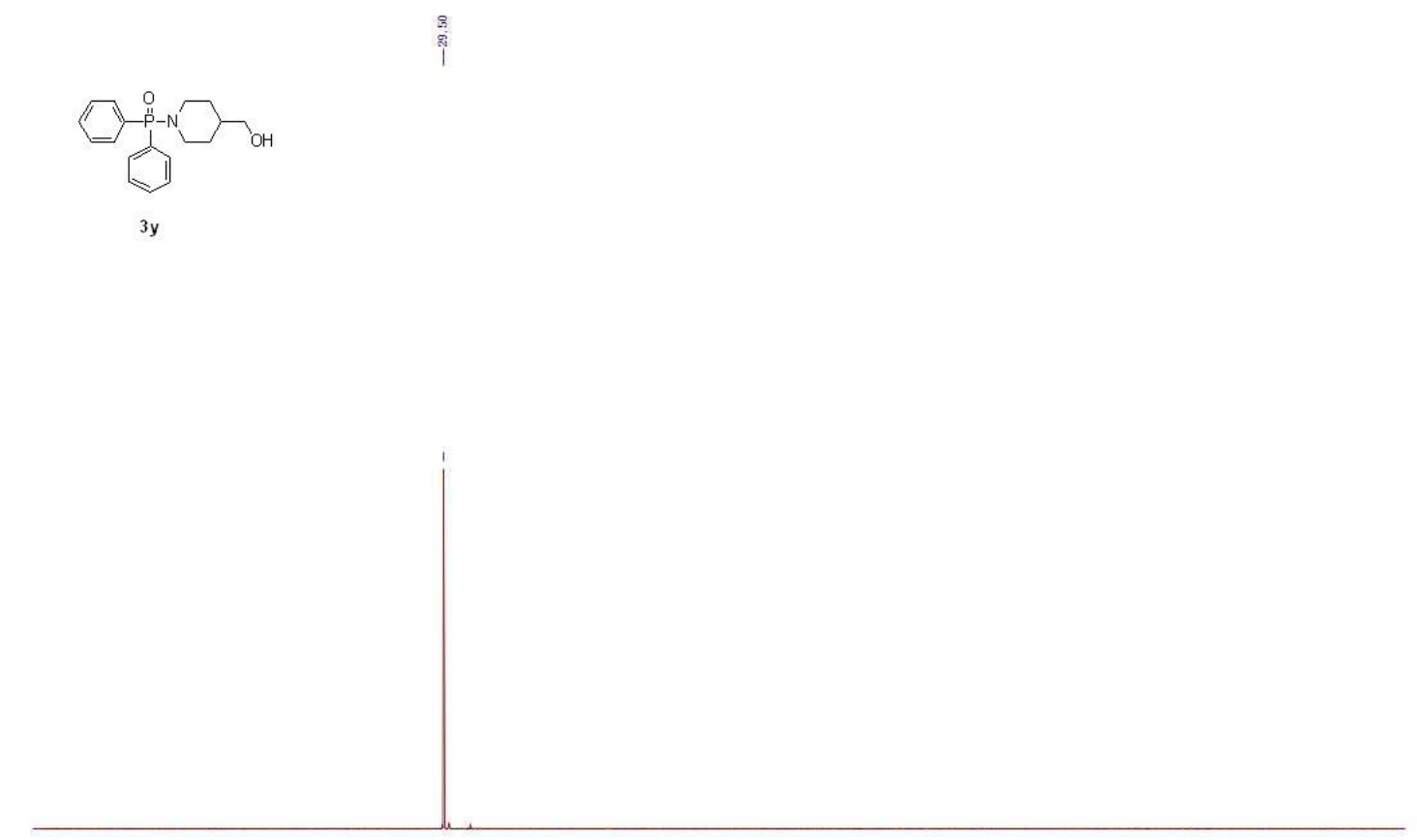

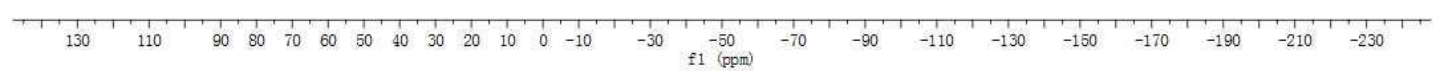

${ }^{1} \mathrm{H}$ NMR of $\mathbf{3 z}$
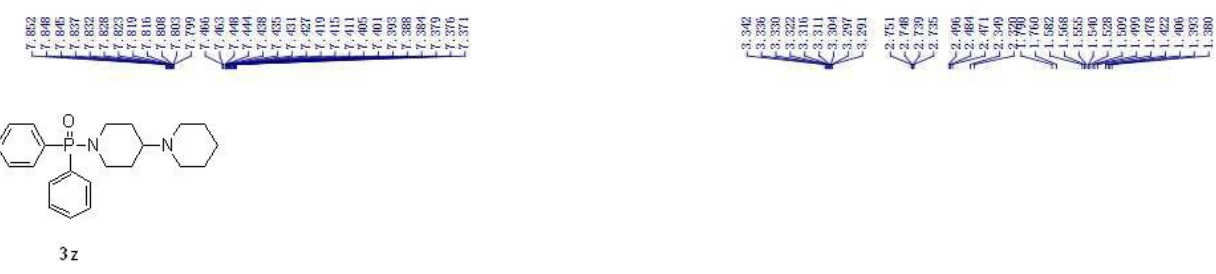

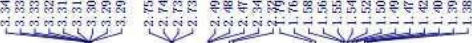

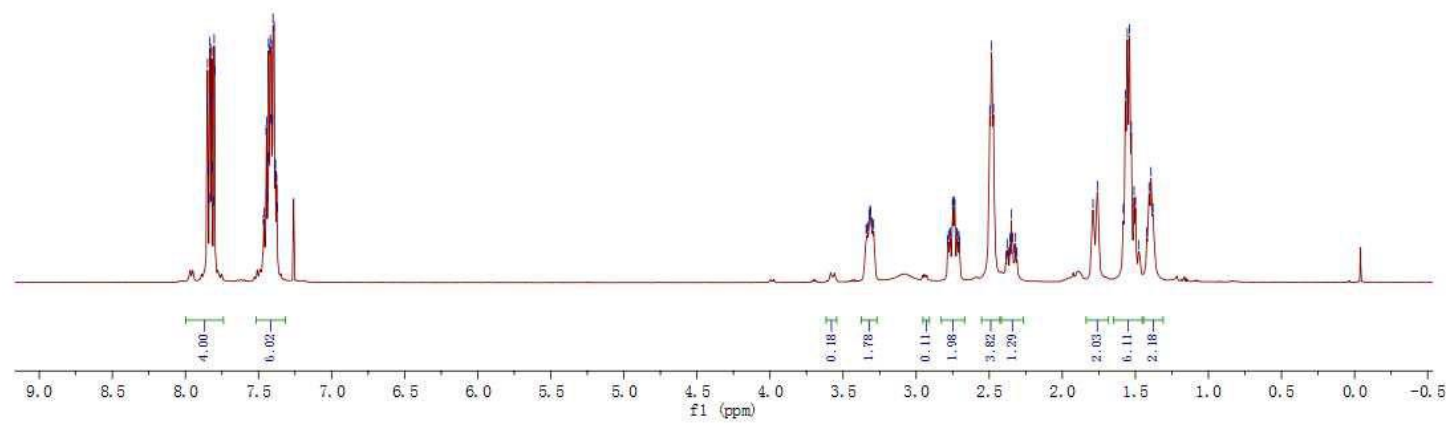


${ }^{13} \mathrm{C}$ NMR of $\mathbf{3 z}$

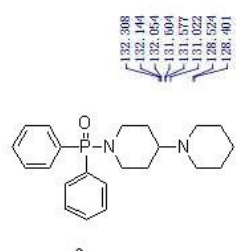

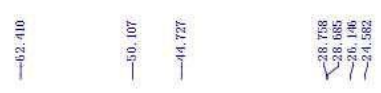

$3 z$
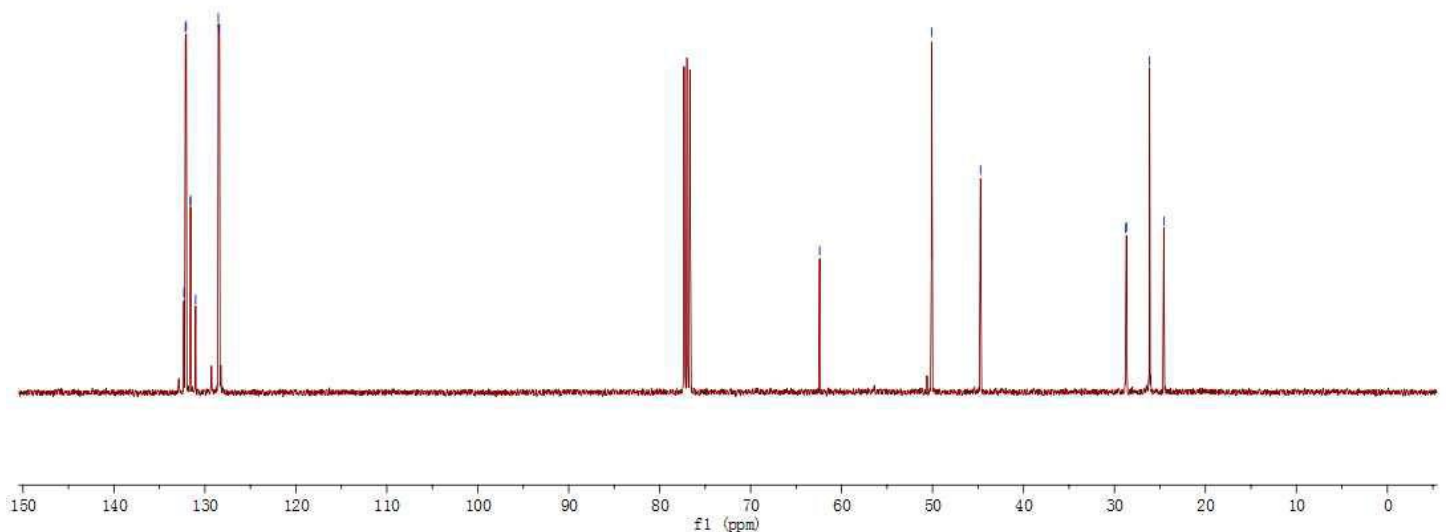

${ }^{31}$ P NMR of $\mathbf{3 z}$

\section{幽}

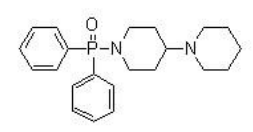

$3 z$

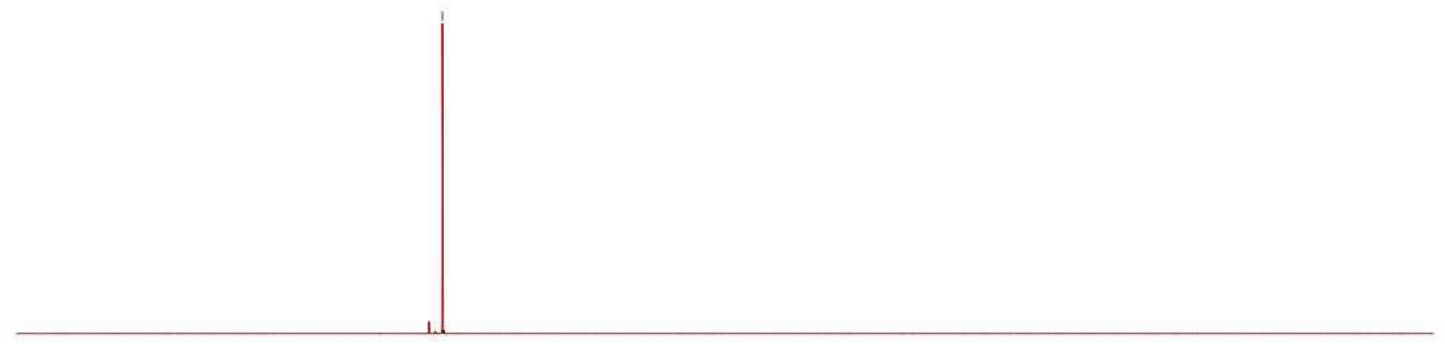

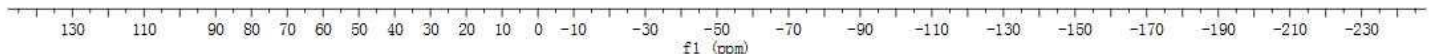




\section{${ }^{1} \mathrm{H}$ NMR of 3A}

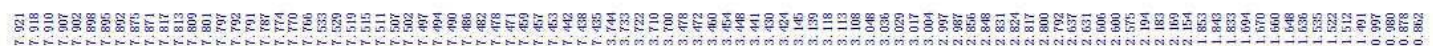

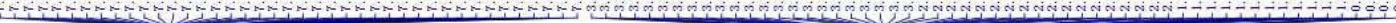
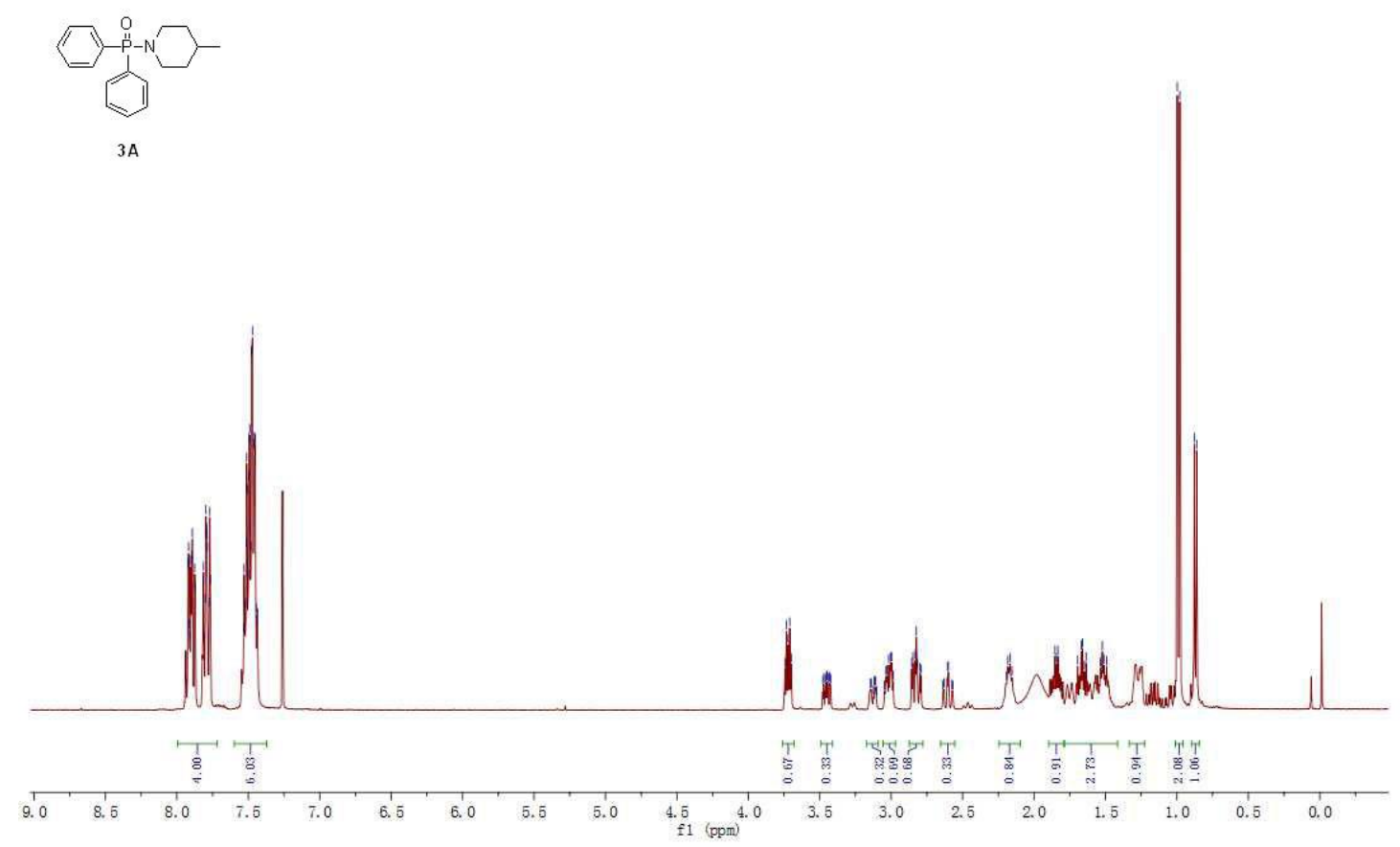

${ }^{13} \mathrm{C}$ NMR of 3A

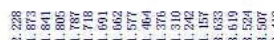

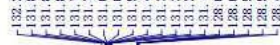

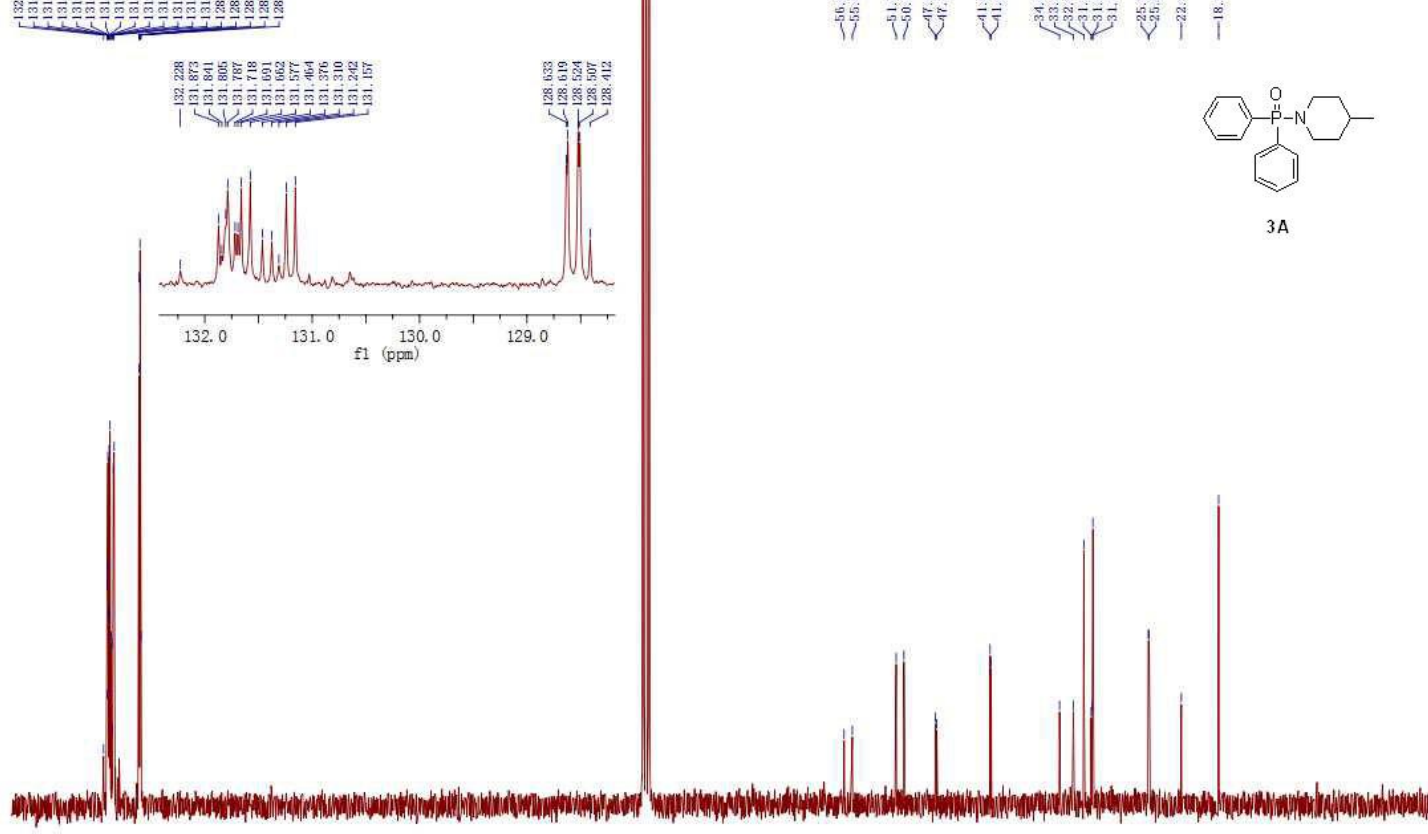

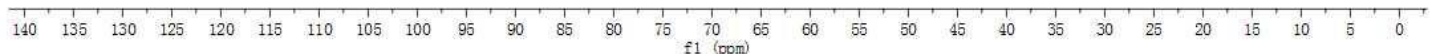


${ }^{31}$ P NMR of $\mathbf{3 A}$
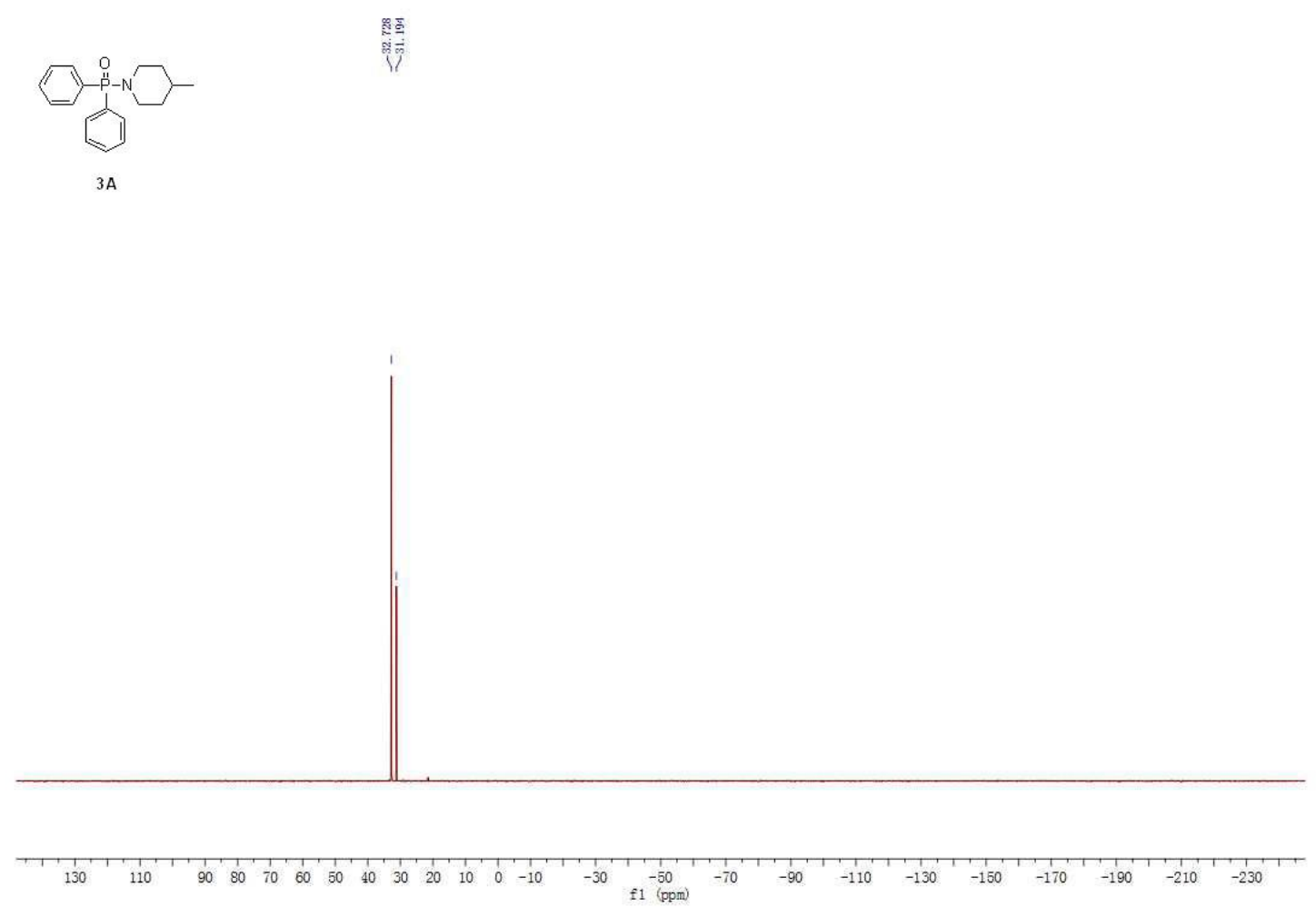

${ }^{1} \mathrm{H}$ NMR of $\mathbf{3 A}\left(d_{6}\right.$-DMSO)

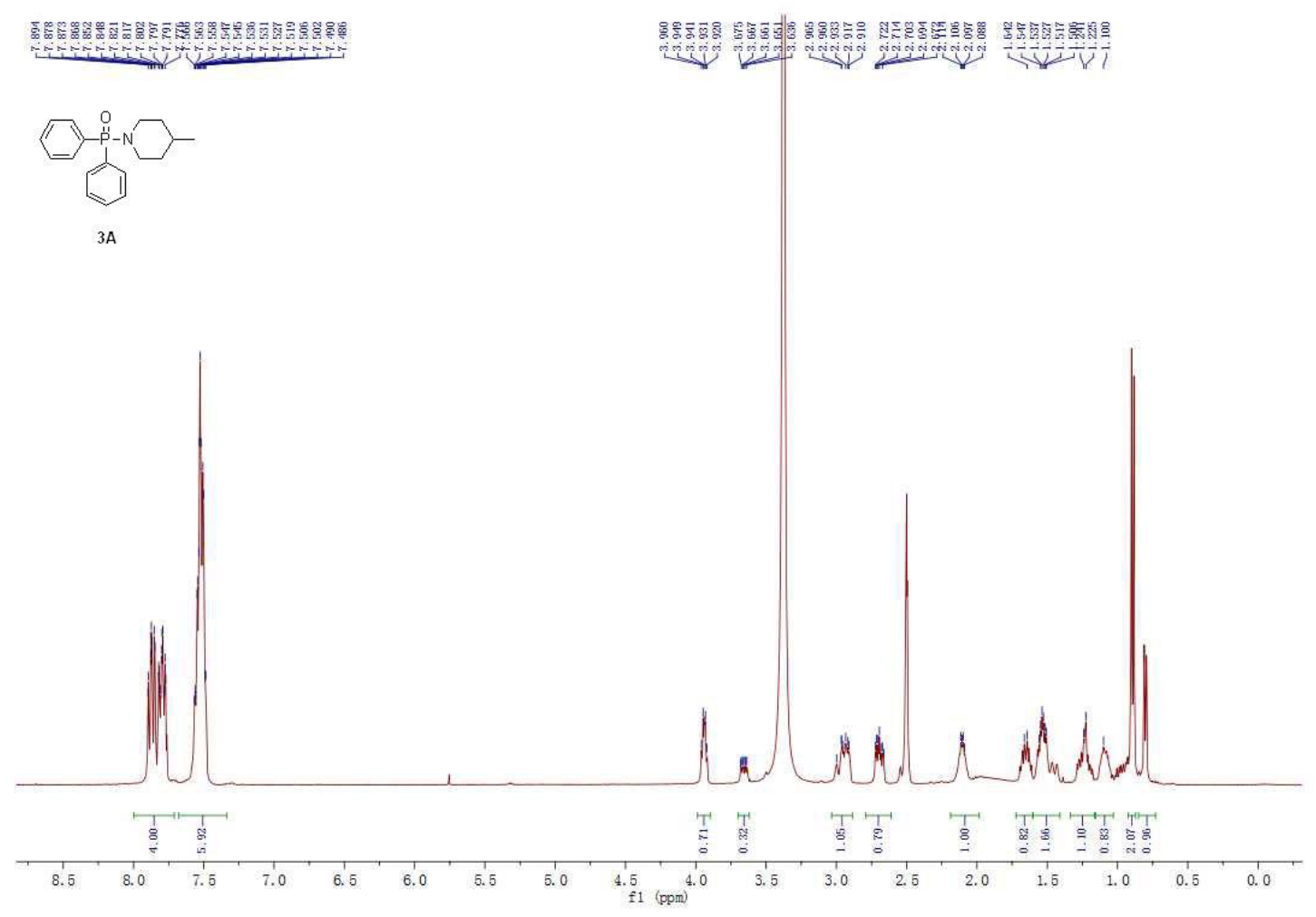


${ }^{1} \mathrm{H}$ NMR of $\mathbf{3 A}\left(d_{6}\right.$-DMSO at $\left.100^{\circ} \mathrm{C}\right)$

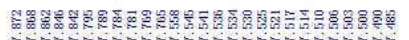

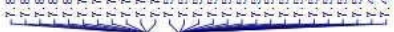

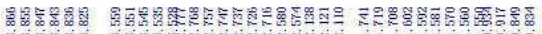
\%

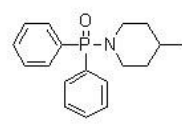

$3 \mathrm{~A}$
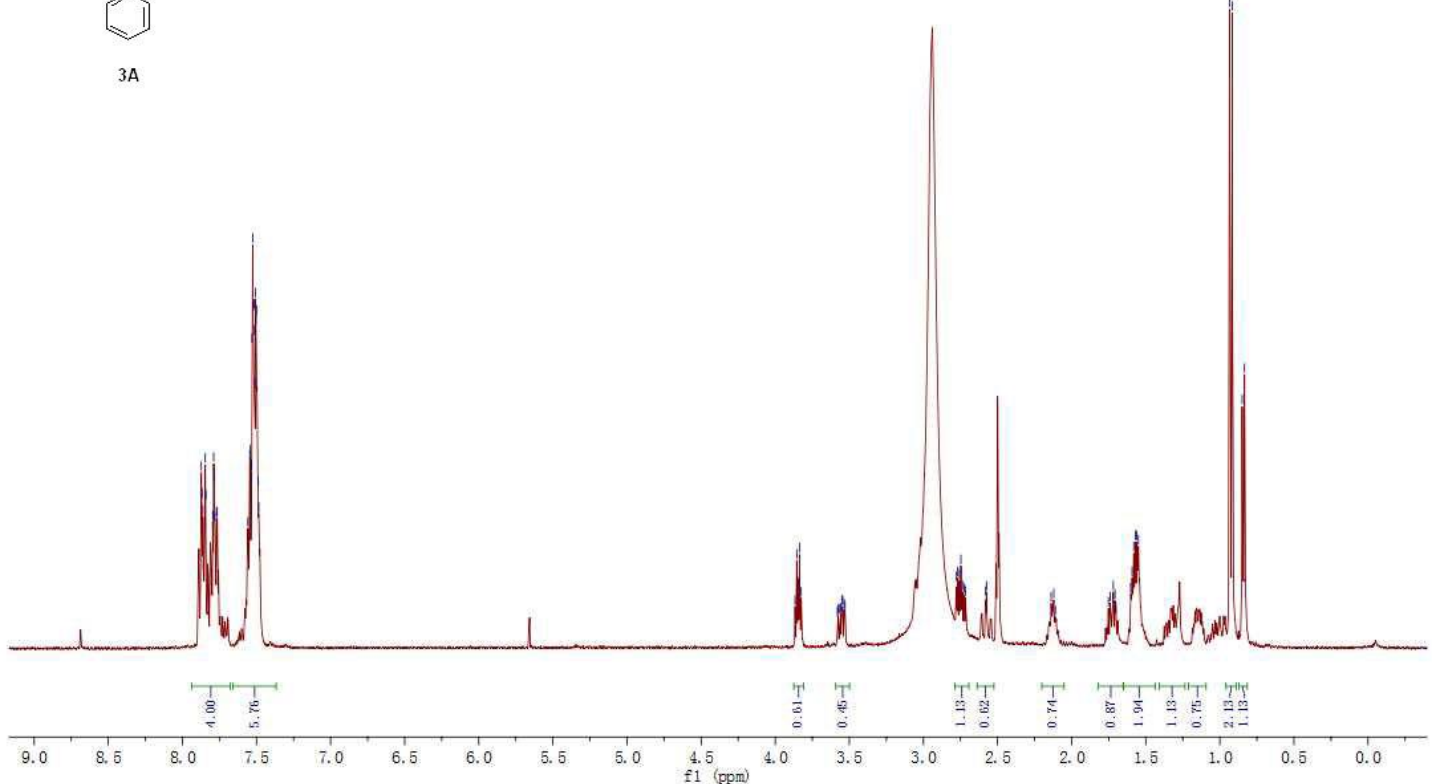

${ }^{1} \mathrm{H}$ NMR of 3B

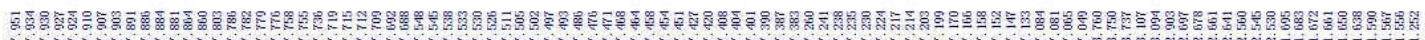<smiles>[B]C1CCN(P(=O)(c2ccccc2)c2ccccc2)CC1</smiles>

$3 \mathbf{B}$

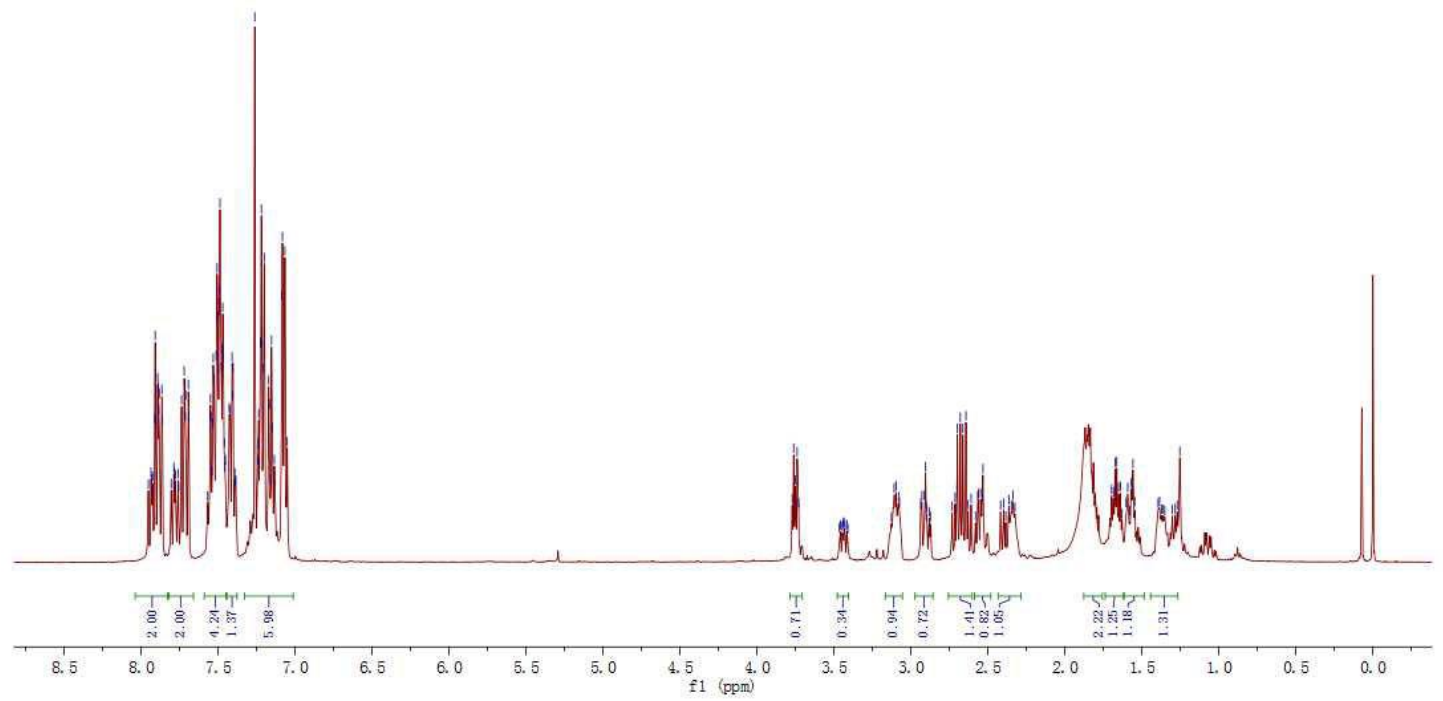


${ }^{13} \mathrm{C}$ NMR of 3B
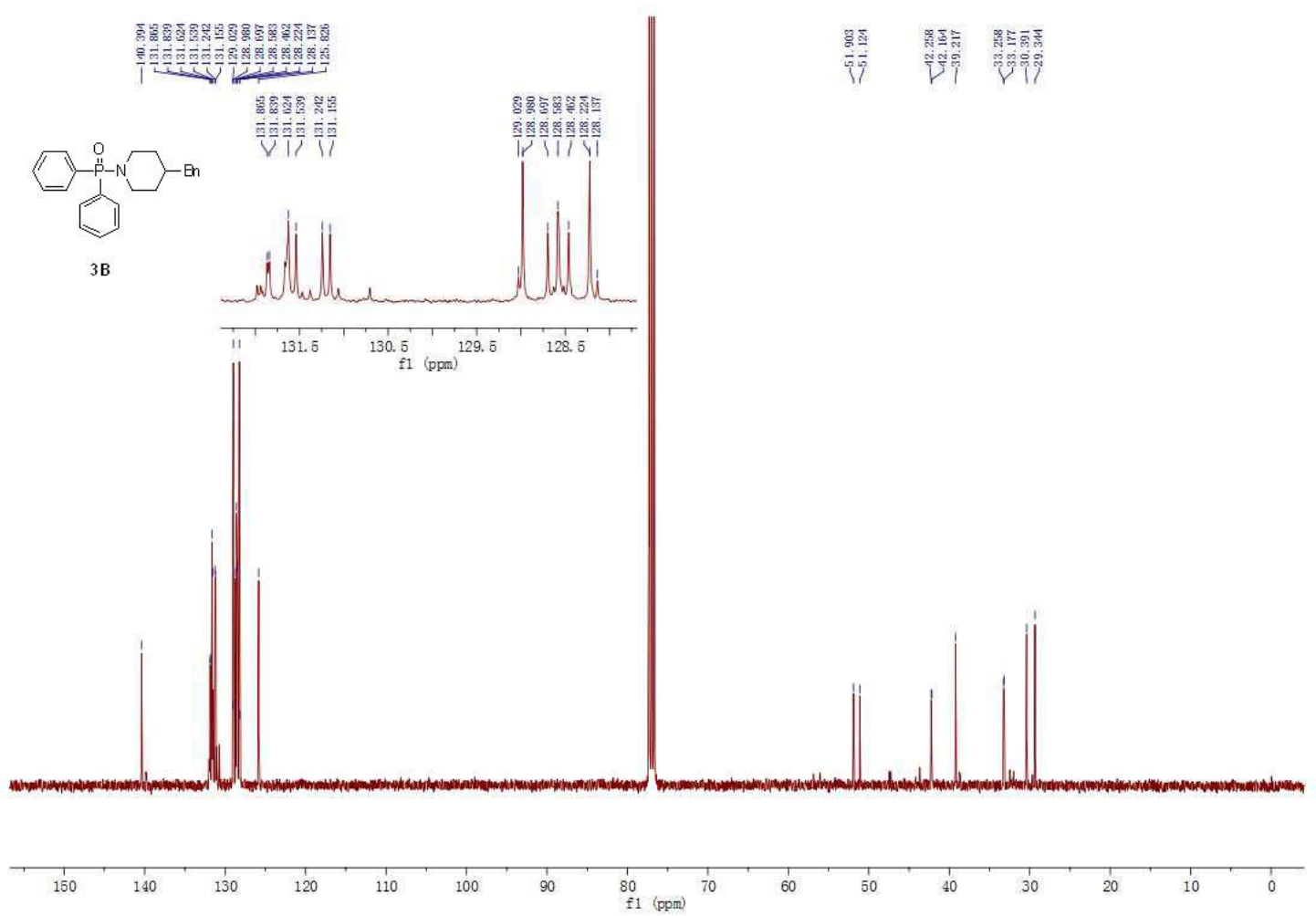

${ }^{31} \mathrm{P}$ NMR of 3B<smiles>c1ccc2c(c1)CC[C+]21CCCCC1</smiles>

3 B

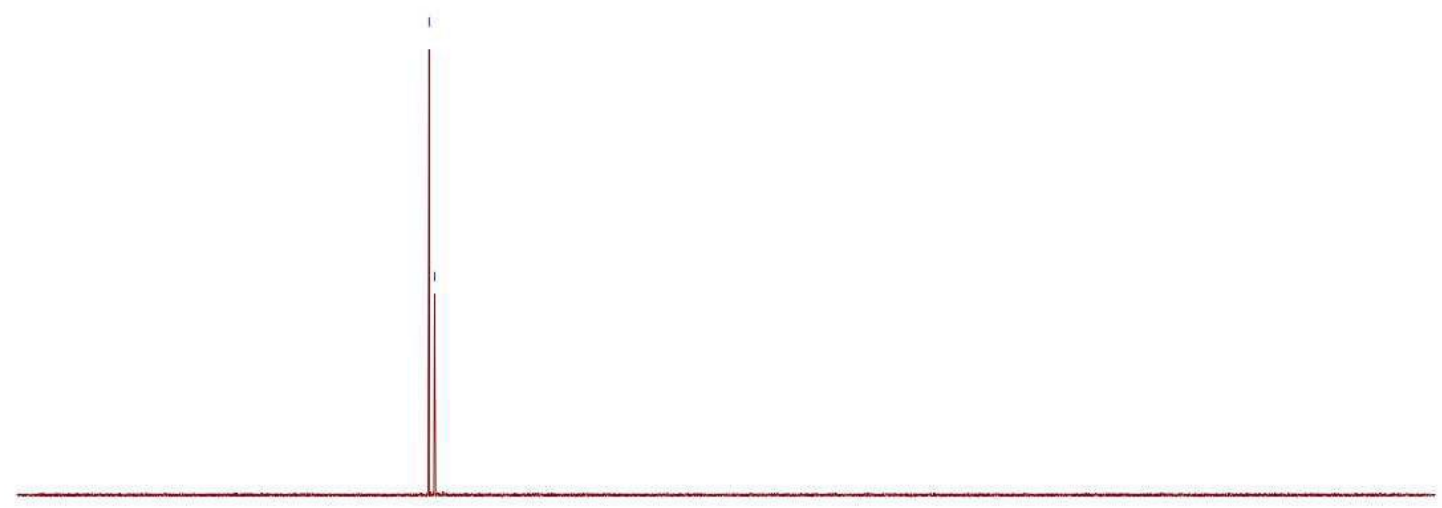

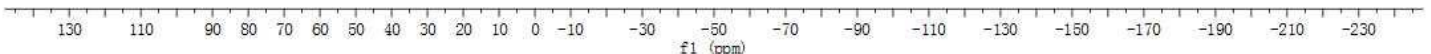

